\title{
Medication Supports at Transitions Between Hospital and Other Care Settings: A Rapid Scoping Review
}

\author{
Shawn Varghese $\mathbb{D}^{1-3}$, Shoshana Hahn-Goldberg $\mathbb{D}^{1,4}$, ZhiDi Deng $\mathbb{D}^{1}$, Glyneva Bradley-Ridout $\mathbb{D}^{1,5}$, \\ Sara JT Guilcher $\mathbb{D}^{1,6}$, Lianne Jeffs $\mathbb{D}^{6-8}$, Craig Madho $\mathbb{D}^{4}$, Karen Okrainec $\mathbb{D}^{9}$, Zahava RS Rosenberg-Yunger $\mathbb{D}^{10}$, \\ Lisa M McCarthy $\mathbb{1 D}^{1,2,11,12}$
}

'Leslie Dan Faculty of Pharmacy, University of Toronto, Toronto, Ontario, Canada; ${ }^{2}$ Women's College Research Institute, Women's College Hospital, Toronto, Ontario, Canada; ${ }^{3}$ Michael G.Degroote School Of Medicine, McMaster University, Hamilton, Ontario, Canada; ${ }^{4}$ OpenLab, University Health Network, Toronto, Ontario, Canada; ${ }^{5}$ Gerstein Science Information Centre, University of Toronto, Toronto, Ontario, Canada; ${ }^{6}$ Institute for Health Policy Management and Evaluation, University of Toronto, Toronto, Ontario, Canada; ${ }^{7}$ Sinai Health, Toronto, Ontario, Canada; ${ }^{8}$ Lawrence S. Bloomberg Faculty of Nursing, University of Toronto, Toronto, Ontario, Canada; ${ }^{9}$ Toronto General Research Institute, University Health Network, Toronto, Ontario, Canada; ${ }^{10}$ Ted Rogers School of Management, School of Health Services Management, Ryerson University, Toronto, Ontario, Canada; "'Temerty Faculty of Medicine, University of Toronto, Toronto, Ontario, Canada; ${ }^{12}$ Institute for Better Health, Trillium Health Partners, Mississauga, ON, Canada

Correspondence: Lisa M McCarthy, Clinician Scientist, Institute for Better Health, Trillium Health Partners, Tel +I 4I6-566-2793, Email lisa.mccarthy@utoronto.ca

Purpose: Transitions in care (TiC) often involves managing medication changes and can be vulnerable moments for patients. Medication support, where medication changes are reviewed with patients and caregivers to increase knowledge and confidence about taking medications, is key to successful transitions. Little is known about the optimal tools and processes for providing medication support. This study aimed to identify describe patient or caregiver-centered medication support processes or tools that have been studied within 3 months following TiC between hospitals and other care settings.

Methods: Rapid scoping review; English-language publications from OVID MEDLINE, OVID EMBASE, Cochrane Library and EBSCO CINAHL (2004-July 2019) that assessed medication support interventions delivered within 3 months following discharge were included. A subset of titles and abstracts were assessed by two reviewers to evaluate agreement and once reasonable agreement was achieved, the remainder were assessed by one reviewer. Eligibility assessment for full-text articles and data charting were completed by an experienced reviewer.

Results: A total of 7671 unique citations were assessed; 60 studies were included. Half of the studies $(\mathrm{n}=30 / 60)$ were randomized controlled trials. Most studies $(n=45 / 60)$ did not discuss intervention development, particularly whether end users were involved in intervention design. Many studies $(n=37 / 60)$ assessed multi-component interventions with written/print and verbal education components. Few studies $(n=5 / 60)$ included an electronic component. Very few studies $(n=4 / 60)$ included study populations at high risk of adverse events at TiC (eg, people with physical or intellectual disabilities, low literacy or language barriers).

Conclusion: The majority of studies were randomized controlled trials involving verbal counselling and/or physical document delivered to the patient before discharge. Few studies involved electronic components or considered patients at high-risk of adverse events. Future studies would benefit from improved reporting on development, consideration for electronic interventions, and improved reporting on patients with higher medication-related needs.

Keywords: continuity of patient care, patient discharge, patient education, rapid scoping review, medication counseling, care transitions

\section{Introduction}

The World Health Organization (WHO) has identified transitions in care (TiC) as a key focus area for improving patient safety and minimizing preventable medication-related harm. ${ }^{1} \mathrm{TiC}$, in which patients move from one care setting to 
another, often involves changes to medication regimens. ${ }^{2}$ During transitions, discrepancies and gaps in communication can lead to medication errors, ${ }^{3}$ which in turn, can cause patient harm and lead to emergency department visits, hospitalization, and even death. ${ }^{4}$ A study assessing patient's recall of medication changes one week after discharge found less than half were able to recall whether and which medications changed during their hospital stay, and that nearly $10 \%$ experienced changes in their perceived informational needs during their care transition. ${ }^{5}$ As inadequate knowledge about medication regimens can lead to incorrect use and non-adherence, ${ }^{6}$ these findings suggest that many patients may be at risk of harm and/or suboptimal therapy following discharge. Furthermore, certain patient groups are at higher risk for adverse events during transitions in case. Patients with language barriers or low literacy levels often have difficulty understanding their discharge medications. ${ }^{7,8}$ Older people and those with intellectual or physical disabilities experience high rates of multimorbidity and are often prescribed many medications, which need to be reviewed frequently. ${ }^{9-11}$

Medication support interventions are key to successful transitions. ${ }^{12}$ We define medication support interventions as those in which patients and caregivers partner with health-care providers to review medication changes with the goal of increasing the patients' knowledge and confidence about taking their medications. Current knowledge and understanding of the optimal tools for providing medication support during TiC is limited. Further study is required to understand both the design and implementation of successful support tools. As part of this effort, we performed a rapid scoping review to describe medication support tools and processes that have been studied, understand the extent to which elements of usercentered design were included in their development, and to identify opportunities for future research. This rapid scoping review is a component of a multi-method research project to understand the needs and co-develop guidelines and prototypes of potential medication support tool(s) together with patients and providers, with the goals of improving people's medication experiences and optimizing medication management at TiC.

\section{Objective}

This rapid scoping review sought to address the following research question: What patient-centered or caregiver-centered medication support tools or processes have been studied within three months following TiC between hospitals and other care settings?

\section{Methods}

We undertook a rapid scoping review with the intent of identifying evidence in a timely fashion to facilitate the integration of our findings into our complementary co-design work to create medication support tools for use during TiC. ${ }^{13}$ Our methods were guided by the information and recommendations outlined in the Joanna Briggs Institute Manual for Evidence Synthesis on scoping reviews. ${ }^{14}$ Reporting of our work was guided by the Preferred Reporting Items for Systematic Reviews and Meta-analysis for Scoping Reviews (PRISMA-ScR). ${ }^{15}$

\section{Eligibility Criteria}

Study eligibility criteria were as follows.

P - Population: We included studies that involved patients experiencing $\mathrm{TiC}$, and their caregivers. Caregivers were defined as any person who gives care to people who need help taking care of themselves. Examples included family members, friends, or members of the clergy. ${ }^{16}$ Only patients experiencing transitions from hospitals to other care settings (home/community, primary care, long-term care, rehabilitation facilities, etc.) were included because this represents a population at high risk for experiencing medication errors and adverse events. ${ }^{17,18}$

I-Intervention: Studies that involved or assessed a medication support intervention were included. A medication support intervention was defined as any process or tool that aims to increase patients and caregivers' understanding of their medications, confidence about taking their medication, or aims to increase providers' understanding of a patient's preferences and values with respect to their medications. Broader interventions, such as discharge summaries, with medication information were included.

C - Comparator: Studies with or without controls were eligible for inclusion.

O - Outcomes: Studies that assessed patient and caregivers' knowledge/comprehension, patient attitudes (satisfaction, preferences, values) and confidence (self-efficacy), patient behaviour (self-management, adherence) and health 
system outcomes (health care utilization (eg, physician visits, pharmacy visits, readmission) and mortality) were included. Only studies that measured outcomes within 3 months of discharge were included because this time frame is frequently cited as the period in which the outcome may be associated with the intervention administered at discharge. ${ }^{19}$

S - Study Designs: Eligible study designs included: experimental studies (randomized controlled trials, quasiexperimental studies with control(s)), observational studies (prospective and historical cohort, case-control, case-crossover, case-cohort, case-time controlled, self-controlled case-series design, cross-sectional studies, time-series, pre-post studies), qualitative studies, and mixed methods studies. ${ }^{20}$ Descriptive quantitative studies were excluded as these studies do not assess the effectiveness of the intervention. We included published or in-press peer reviewed articles published as full reports. Book chapters, abstracts, conference proceedings were excluded.

Report Characteristics: Given the short time to conduct the review, we confined our search to published literature available in English-language only. We limited eligible studies to those published within the previous 15 years (2004 -2019) due to rapid shifts in clinical practice supporting medication management.

\section{Literature Search Strategy}

The search strategy was developed and executed by a university-affiliated librarian co-investigator (Appendix 1). A second librarian with expertise in health literature reviewed the search strategies using the PRESS EBC Checklist. ${ }^{21}$ The following databases were searched between July 26 and 30, 2019:

- Ovid MEDLINE (1946-present including Epub ahead of print, in-process, and other unindexed citations) Ovid MEDLINE (1946-present including Epub ahead of print, in-process, and other unindexed citations),

- Ovid EMBASE (1947-present),

- Cochrane Library,

- EBSCO CINAHL Plus with Full Text (1981-present).

Search results were exported by the librarian into EndNote and duplicates were removed. After duplicate removal, citations from only the prior 15 years (ie, 2019 to 2004) were identified.

\section{Study Selection}

Citation titles and abstracts from the searches were screened to determine study eligibility within Covidence ${ }^{\circledR}{ }^{22}$ Interrater agreement was assessed, and any conflicts resolved.

Standardized relevance screening forms with instructions were prepared and piloted to ensure uniform application of eligibility criteria. Titles and abstracts of identified references were reviewed by an experienced member of the study team. A second reviewer verified a subset of the records (5\%) examined by the first reviewer and was also consulted for citations about which the first reviewer is uncertain (DG). Agreement on this subset of records was above a minimum kappa value of 0.80 taken to represent substantial agreement. ${ }^{23}$

Full-text articles were retrieved and reviewed using the process described above for any abstract that required further clarification or was related to the study research question. Disagreements were discussed until consensus was reached or reconciled by a third reviewer.

Team members did not review studies they authored, co-authored or consulted upon.

A review log was created to document all decisions made in relation to selecting studies for inclusion including recommendations of the reviewer(s) and reason(s) for exclusion to facilitate development of a flowchart, recommended by PRISMA. ${ }^{24}$

\section{Data Charting Process and Data Items}

We collected data about study characteristics (eg study design, citation), population characteristics (eg number of patients, age, gender, whether the population included was at high risk for adverse events due to presence of disabilities or language barriers), intervention characteristics (eg physical, verbal, or electronic modes of delivery, components of intervention such as videos, counselling, medication list, follow-up, content of intervention, layout (design), process of 
the interventions, transition type), study outcomes (eg patient and caregiver knowledge/comprehension, patient and caregiver attitudes and confidence, patient and caregiver behaviour and experience, health system outcomes, other outcomes), and conclusions.

Fields for data abstraction were based on those recommended by the Cochrane Collaboration and the Centre for Reviews and Dissemination. ${ }^{24,25}$ We were also guided by a systematic review conducted by members of our study team that explored the impact of patient-centered discharge tools. ${ }^{19}$ Further, the extent of user-centered design approaches used to develop each tool was assessed with the UCD-11 (User-Centred Design for Patient-Centred Tools) instrument. ${ }^{26}$ The UCD-11 instrument was used to generate a descriptive measure of an intervention's user-centeredness through assessing the extent of end user (eg patients, caregivers, health professionals) involvement in the design, development, and refinement stages of the intervention. ${ }^{26}$ If the criteria was not explicitly reported in the article, it was assumed this criteria was not a part of tool development.

A preliminary data abstraction form and accompanying guide were created. Cochrane's process for developing the data extraction form guided this process: a paper form was designed, two reviewers independently extracted data from 10 studies, and then met to determine the necessary revisions. Feedback was used to modify the form and repeat the pilot before creating the form electronically. Concurrently, a guide with instructions for completing the extraction and decision rules for coding data was created and revised iteratively. Once the fields for extraction and their accompanying coding rules were agreed upon, an electronic form was developed to facilitate data entry and management.

Due to the time constraints in which this rapid scoping review was conducted, one experienced reviewer abstracted data . A second reviewer was consulted in cases of uncertainty. Disagreements that could not be resolved through consensus were reconciled through discussion with the two reviewers in the presence of a third person .

Mechanisms for recording, assessing, and correcting data entry errors were devised.

A descriptive numerical summary and a narrative synthesis were completed to describe study designs, intervention (and comparator intervention, if applicable) characteristics, target population characteristics, and study outcomes, as stated in our study objective. Results were classified based on main conceptual categories (eg timing, mode of delivery, health-care providers involved). Summary tables were created for study characteristics, intervention characteristics, and development of the interventions (eg UCD-11).

\section{Risk of Bias in Individual Evidence Sources}

Our primary interest in this body of literature was to create an inventory of design, layout, and process considerations. Consistent with established scoping review methods, we did not appraise the risk of bias for included studies. ${ }^{14,15}$

\section{Results}

\section{Search Results}

The search yielded 8231 reports (Figure 1). After removing duplicates, 7671 articles were assessed for eligibility and after title and abstract screening, 113 articles were marked for full-text review. After full-text review, 53 articles were excluded, and 60 articles remained eligible. ${ }^{27-86}$ For a large number of articles $(n=45 / 53)$, the full text could not be found. Other reasons for excluding studies after full-text review included ineligible design $(n=3)$ or outcome $(n=3)$ and duplicates $(\mathrm{n}=1)$ that were not identified during the title and abstract screening stage. None of the included studies were co-authored by a team member.

\section{Study Characteristics}

Of the 60 studies included, $50.0 \%(\mathrm{n}=30)$ were randomized controlled trials, ${ }^{28,29,33-36,39,43,45-47,50,53,54,61-64,68-74,76,80,83,84,86}$ $23.3 \%(\mathrm{n}=14)$ were non-randomized trials, ${ }^{30,31,37,40,48,49,51,56-58,60,75,77,78} 11.7 \%(\mathrm{n}=7)$ were cohort studies, ${ }^{27,42,52,59,66,67,79}$ $8.3 \%(\mathrm{n}=5)$ were before-and-after studies, ${ }^{32,41,55,81,82} 5.0 \%(\mathrm{n}=3)$ were quasi-randomized trials, ${ }^{38,44,85}$ and $1.7 \%(\mathrm{n}=1)$ were case-control studies. ${ }^{65}$ The majority of studies $(53.3 \%, \mathrm{n}=32 / 60)$ were conducted in the United States of America (USA). ${ }^{29,30,32-34,37,39,42,43,45,48,49,51,53,57,59-61,64-67,69-71,75,78,79,81-83,85}$ Other countries of origin for more than one study included Australia $(\mathrm{n}=4),{ }^{38,40,55,84}$ Canada $(\mathrm{n}=4),{ }^{44,47,76,80}$ Brazil $(\mathrm{n}=2),{ }^{36,68}$ Croatia $(\mathrm{n}=2),{ }^{62,63}$ France $(\mathrm{n}=2),{ }^{56,72}$ 


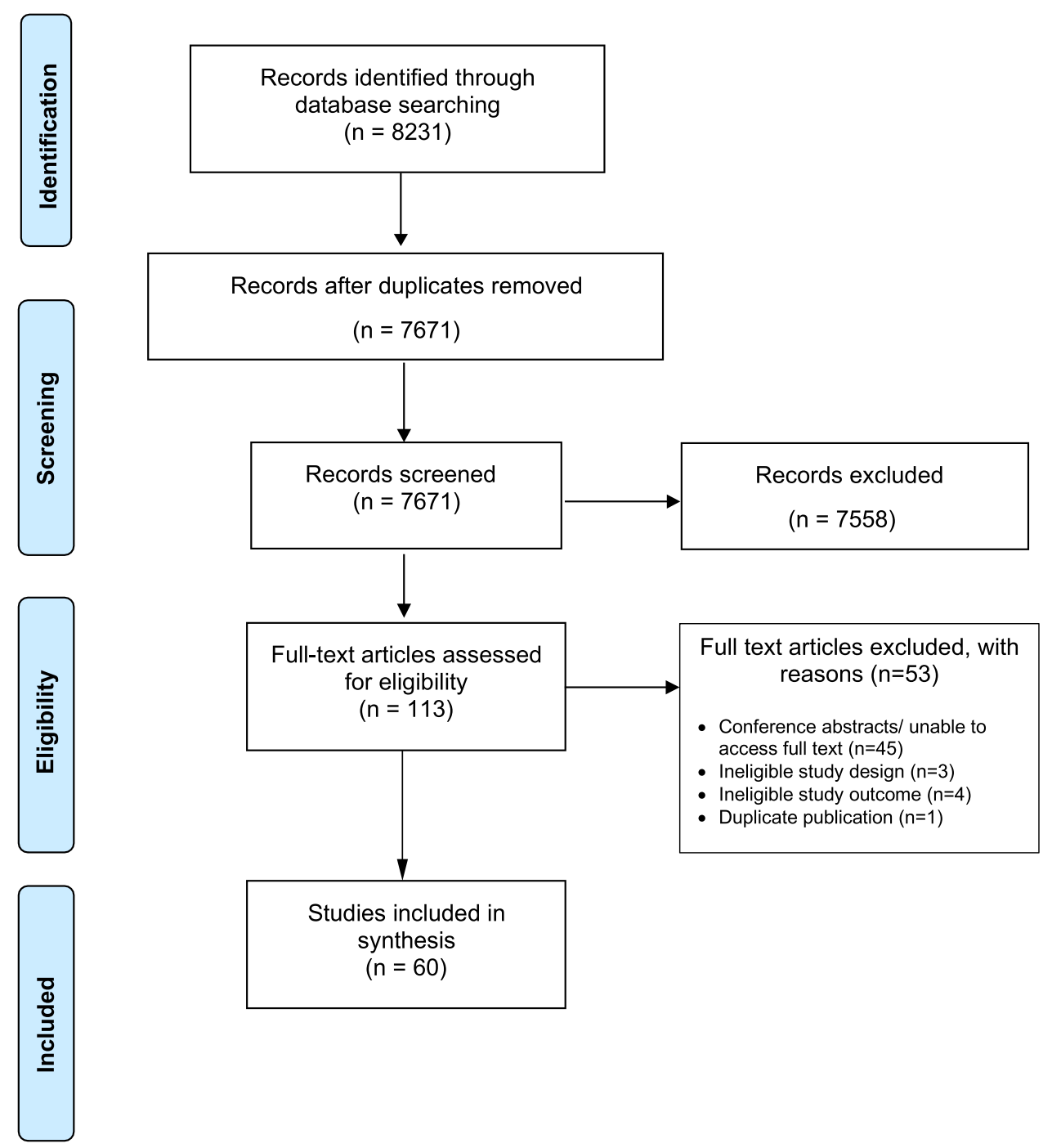

Figure I PRISMA flow diagram.

and Switzerland $(\mathrm{n}=2){ }^{52,58}$ The remaining 12 studies originated from other unique countries. Table 1 describes the participants, intervention, comparator and outcome(s) of the included studies. Table 2 provides additional information regarding the interventions of included studies ie, specifies health-care providers delivering the intervention, the timing of the intervention, the various components of multi-pronged interventions, etc.

Many studies selected patients based on age $(76.7 \%, \mathrm{n}=46 / 60)^{27-30,33-45,47,49-53,55-57,60-64,66,67,71-76,78,80,81,83-86}$ and presence of a particular medical condition $(50.0 \%, \mathrm{n}=30 / 60) .{ }^{29-31,33,34,36,37,40,43-45,47,48,50,53,54,59,60,63,66-69,71,76,78,82-84,86}$ Fewer studies selected patients based on the medication-class prescribed $(30.0 \%, \mathrm{n}=18 / 60)^{29,30,39-41,44,47,48,53,64,68-}$ $71,74,78,84,85$ or the patients' number of medications $(31.7 \%, \mathrm{n}=19 / 60) .{ }^{28,30,33,35,43,46,48,49,52,55,58,61,62,65,70,74,77,84,85}$ Few studies selected patients based on the presence of an intellectual or physical disability $(3.3 \%, n=2 / 60),{ }^{45,84}$ low literacy level $(n=1 / 60),{ }^{48}$ or language barrier $(n=1 / 60) .{ }^{84}$ There were also only a few studies that included a subgroup analysis involving patients with low literacy $(8.3 \%, \mathrm{n}=5 / 60),{ }^{34,38,53,69,71}$ intellectual or physical disability $(5.0 \%, \mathrm{n}=3 / 60),{ }^{34,45,84}$ and language barriers $(\mathrm{n}=1 / 60){ }^{84}$

\section{UCD-I I Reporting}

Only $25 \%$ of studies $(n=15 / 60)$ explicitly discussed the development of the intervention, ${ }^{29,31,33-36,43,47,51,52,64,76,81,82,86}$ making it difficult to apply the UCD-11 criteria. Of the 60 articles, none met all 11 criteria and only 1 met 10 of the 11 
Table I Summary of Studies Included

\begin{tabular}{|c|c|c|c|c|}
\hline $\begin{array}{l}\text { Study, Year, } \\
\text { Country } \\
\text { Design }\end{array}$ & $\mathbf{N}$ and Study Population & Intervention(s) & Comparator & Outcome \\
\hline $\begin{array}{l}\text { Acomb et al, } \\
2013^{27} \\
\text { United } \\
\text { Kingdom } \\
\text { Cohort study }\end{array}$ & $\begin{array}{l}\text { I } 365 \text { older adults discharged from } \\
\text { older people admissions ward }\end{array}$ & $\begin{array}{l}\text { Medicines Care Plan added to } \\
\text { discharge communication. Patients } \\
\text { were also signposted to primary } \\
\text { care provider for follow-up if } \\
\text { needed. }\end{array}$ & No Medicines Care Plan & -Readmission rate ${ }^{\mathrm{a}}$ \\
\hline $\begin{array}{l}\text { Al-Hashar } \\
\text { et al, } 2018^{28} \\
\text { Oman } \\
\text { Randomized } \\
\text { controlled } \\
\text { trial }\end{array}$ & $\begin{array}{l}587 \text { adults taking I or more } \\
\text { medications }\end{array}$ & $\begin{array}{l}\text { Pharmacist performed medication } \\
\text { reconciliation and identified } \\
\text { medication discrepancies on } \\
\text { admission and at discharge. } \\
\text { Medication dispensed at bedside } \\
\text { with detailed instructions and } \\
\text { take-home medication list. }\end{array}$ & $\begin{array}{l}\text { Pharmacist performed standard } \\
\text { medication history review on } \\
\text { admission, and medications } \\
\text { dispensed at pharmacy window } \\
\text { with basic instructions. }\end{array}$ & $\begin{array}{l}\text {-Rates of preventable adverse drug } \\
\text { events } 30 \text { days post-discharge }{ }^{\mathrm{a}} \\
\text {-Hospitalization due to a } \\
\text { preventable adverse drug event } \\
(\mathrm{ADE})^{\mathrm{a}} \\
- \text { Nonadherence associated with } \\
\text { ADE }^{\mathrm{a}} \\
- \text {-Medication discrepancy } \\
\text { associated with ADE } \\
\text {-Healthcare resource use (eg, } \\
\text { readmissions, emergency } \\
\text { department visits) }\end{array}$ \\
\hline $\begin{array}{l}\text { Anderegg } \\
\text { et al, } 2013^{29} \\
\text { United States } \\
\text { Randomized } \\
\text { controlled } \\
\text { trial }\end{array}$ & $\begin{array}{l}192 \text { adults receiving anticoagulant } \\
\text { therapy or had previous diagnosis } \\
\text { of one of nine cardiovascular (eg } \\
\text { hypertension), respiratory } \\
\text { conditions (eg asthma), or } \\
\text { diabetes mellitus }\end{array}$ & $\begin{array}{l}\text { Pharmacy case managers } \\
\text { performed medication } \\
\text { reconciliation, patient education, } \\
\text { and pharmacotherapy assessment. } \\
\text { At discharge, patient received } \\
\text { education, medication list, and } \\
\text { wallet card with information from } \\
\text { pharmacy case managers. }\end{array}$ & $\begin{array}{l}\text { Usual care with medication } \\
\text { reconciliation performed by unit } \\
\text { pharmacists on admission, and } \\
\text { discharge summary and education } \\
\text { provided by unit nurses. } \\
\text { Medication lists were provided. }\end{array}$ & $\begin{array}{l}\text {-Acceptance rate of pharmacy } \\
\text { case manager recommendations } \\
\text { by inpatient physicians }(48 \%) \\
\text {-Healthcare resource use (eg, } \\
\text { readmissions, urgent care visits) }\end{array}$ \\
\hline $\begin{array}{l}\text { Bailey et al, } \\
2019^{30} \\
\text { United States } \\
\text { Non- } \\
\text { randomized } \\
\text { trial }\end{array}$ & $\begin{array}{l}2235 \text { adults with } \geq 1 \text { ambulatory } \\
\text { care-sensitive chronic conditions } \\
\text { and taking a high-risk medication } \\
\text { or } \geq 6 \text { medications }\end{array}$ & $\begin{array}{l}\text { Patient engagement, medication } \\
\text { reconciliation, medication } \\
\text { management service, discharge } \\
\text { preparation, and intensive follow- } \\
\text { up provided by multidisciplinary } \\
\text { team (including nurses, } \\
\text { pharmacists, and social workers). }\end{array}$ & Usual care (unspecified) & $\begin{array}{l}\text {-Medication adherence } \\
\text {-Rate of primary care visits within } \\
\text { I4 days of discharge } \\
\text {-Preventable hospitalization rate } \\
\text {-Overall hospitalization rate } \\
\text {-30-day readmissions rate } \\
\text {-Rate of emergency department } \\
\text { visit }^{\mathrm{b}} \\
- \text {-Medication expenditure }^{\mathrm{a}}\end{array}$ \\
\hline $\begin{array}{l}\text { Baky et al, } \\
2018^{31} \\
\text { Saudi Arabia } \\
\text { Non- } \\
\text { randomized } \\
\text { trial }\end{array}$ & $\begin{array}{l}578 \text { patients with acute coronary } \\
\text { syndrome or heart failure }\end{array}$ & $\begin{array}{l}\text { Administrative clerks scheduled } \\
\text { follow-up appointments for } \\
\text { patients. Pharmacist provided } \\
\text { medication education. Physicians } \\
\text { were encouraged to enter } \\
\text { discharge order ahead of time to } \\
\text { allow patients to receive discharge } \\
\text { instructions early next day and } \\
\text { staff time to carry out } \\
\text { aforementioned activities. }\end{array}$ & Usual care (not specified) & $\begin{array}{l}\text {-30-day all cause readmission rate } \\
\text {-Likelihood of receiving an }^{\mathrm{b}} \\
\text { appointment }^{\mathrm{a}} \\
\text {-Likelihood of receiving } \\
\text { medication education }^{\mathrm{a}} \\
\text {-Likelihood of timely discharge }^{\mathrm{a}}\end{array}$ \\
\hline
\end{tabular}

(Continued) 
Table I (Continued).

\begin{tabular}{|c|c|c|c|c|}
\hline $\begin{array}{l}\text { Study, Year, } \\
\text { Country } \\
\text { Design }\end{array}$ & $\mathrm{N}$ and Study Population & Intervention(s) & Comparator & Outcome \\
\hline $\begin{array}{l}\text { Balling et al, } \\
2015^{32} \\
\text { United States } \\
\text { Before-and- } \\
\text { after study }\end{array}$ & $\begin{array}{l}1058 \text { patients discharged from two } \\
\text { inpatient units (adult medical or } \\
\text { surgical) }\end{array}$ & $\begin{array}{l}\text { Transition-of-care pharmacist } \\
\text { reviewed patient information, } \\
\text { performed medication } \\
\text { reconciliation and delivered } \\
\text { discharge counselling. } \\
\text { Pharmacist also coordinated with } \\
\text { outpatient pharmacy to address } \\
\text { financial barriers. }\end{array}$ & $\begin{array}{l}\text { Readmissions and discharges from } \\
\text { control year in which discharging } \\
\text { physician performed medication } \\
\text { reconciliation, and physicians and } \\
\text { nurses delivered routine patient } \\
\text { education. No transition-of-care } \\
\text { pharmacist in control year. }\end{array}$ & $\begin{array}{l}\text {-Readmissions per month } \\
\text {-Discharges per month } \\
\text {-Medication interventions made by } \\
\text { pharmacist at discharge }\end{array}$ \\
\hline $\begin{array}{l}\text { Barnason } \\
\text { et al, } 2010^{33} \\
\text { United States } \\
\text { Randomized } \\
\text { controlled } \\
\text { trial }\end{array}$ & $\begin{array}{l}38 \text { older adults with heart failure } \\
\text { taking five or more medications } \\
\text { routinely with at least one } \\
\text { medication requiring more than } \\
\text { once per day dosing }\end{array}$ & $\begin{array}{l}\text { In addition to standard heart } \\
\text { failure education program, nurse } \\
\text { assessed patients for medication } \\
\text { predisposing characteristics of } \\
\text { medication use, and personalized } \\
\text { interventions (ie hospital } \\
\text { transition modules and } \\
\text { counselling) based on assessment. }\end{array}$ & $\begin{array}{l}\text { Standard heart failure education } \\
\text { program administered by staff } \\
\text { nurses before discharge. Include } \\
\text { education on heart failure, diet, } \\
\text { medications, and signs and } \\
\text { symptoms. }\end{array}$ & $\begin{array}{l}\text {-Medication adherence }{ }^{\mathrm{a}} \\
\text {-Number of recall barriers } \\
\text {-Self-efficacy } \\
\text {-Medication use behaviour skill } \\
\text {-Health-related quality of life for } \\
\text { symptoms and social limitations }{ }^{\mathrm{a}}\end{array}$ \\
\hline $\begin{array}{l}\text { Bell et al, } \\
2016^{34} \\
\text { United States } \\
\text { Randomized } \\
\text { controlled } \\
\text { trial }\end{array}$ & $\begin{array}{l}85 \mathrm{I} \text { adults with acute coronary } \\
\text { syndromes and/or acute } \\
\text { decompensated heart failure }\end{array}$ & $\begin{array}{l}\text { Pharmacists assessed patients for } \\
\text { medication knowledge and } \\
\text { adherence barriers, performed } \\
\text { medication reconciliation, and } \\
\text { provided tailored counselling using } \\
\text { low-literacy adherence aids. Study } \\
\text { coordinators performed follow- } \\
\text { ups and notified pharmacists to } \\
\text { resolve any identified problems. }\end{array}$ & $\begin{array}{l}\text { Standard medication reconciliation } \\
\text { and counselling performed by } \\
\text { nurses, pharmacists, and physicians } \\
\text { involved in the patients' care. } \\
\text { Follow-ups after discharge were } \\
\text { not routine. }\end{array}$ & $\begin{array}{l}\text {-Time to first unplanned } \\
\text { healthcare care event } \\
\text {-Readmission or Emergency room } \\
\text { visits within } 30 \text { days } \\
\text {-Effect of intervention on } \\
\text { unplanned health care utilization in } \\
\text { those with inadequate health } \\
\text { literacy } \\
\text {-Interaction of the treatment } \\
\text { effect with cognitive status }\end{array}$ \\
\hline $\begin{array}{l}\text { Bolas et al, } \\
2004^{35} \\
\text { Northern } \\
\text { Ireland } \\
\text { Randomized } \\
\text { controlled } \\
\text { trial }\end{array}$ & $\begin{array}{l}162 \text { older adults taking } 3 \text { or more } \\
\text { medications daily }\end{array}$ & $\begin{array}{l}\text { Liaison pharmacist performed } \\
\text { several activities: medication } \\
\text { reconciliation, daily patient } \\
\text { education, personalized } \\
\text { medication record sheet, } \\
\text { discharge counselling, } \\
\text { pharmaceutical discharge letter, } \\
\text { and medicines helpline. }\end{array}$ & $\begin{array}{l}\text { Standard clinical pharmacy service } \\
\text { with no discharge counselling. }\end{array}$ & $\begin{array}{l}\text {-Mismatch error rate between } \\
\text { discharge prescription medication } \\
\text { and home medication in 10-14 } \\
\text { days post discharge for I) drug } \\
\left.\text { name }^{\text {a }} \text { 2) frequency of dosing }{ }^{\mathrm{a}} ; 3\right) \\
\text { drug dose } \\
\text {-Patient knowledge of drug } \\
\text { therapy } \\
\text {-Emergency readmission rates }\end{array}$ \\
\hline $\begin{array}{l}\text { Bonetti et al, } \\
2018^{36} \\
\text { Brazil } \\
\text { Randomized } \\
\text { controlled } \\
\text { trial }\end{array}$ & $\begin{array}{l}\text { I33 adults admitted to a } \\
\text { specialized cardiology ward due to } \\
\text { stable angina, acute coronary } \\
\text { syndrome, congestive heart } \\
\text { failure, valvular disease, } \\
\text { arrhythmias, or hypertension }\end{array}$ & $\begin{array}{l}\text { In addition to usual care, pharmacy } \\
\text { residents provided individual } \\
\text { counselling sessions and } \\
\text { information leaflets at discharge. } \\
\text { Follow-up counselling was also } \\
\text { provided. }\end{array}$ & $\begin{array}{l}\text { Usual care from pharmacists and } \\
\text { healthcare providers; received } \\
\text { pharmaceutical interventions } \\
\text { during hospitalization as needed. }\end{array}$ & $\begin{array}{l}\text {-Mortality rate } \\
\text {-Hospital readmissions related to } \\
\text { heart disease }^{\mathrm{a}} \\
\text {-Overall readmissions and } \\
\text { emergency department visits } \\
\text { related to heart disease } \\
\text { Medication adherence }\end{array}$ \\
\hline $\begin{array}{l}\text { Budiman et al, } \\
2016^{37} \\
\text { United States } \\
\text { Non- } \\
\text { randomized } \\
\text { trial }\end{array}$ & I35 adults with STEMI & $\begin{array}{l}\text { Pharmacist provided medication } \\
\text { reconciliation, education, } \\
\text { counselling, and follow-up. } \\
\text { Medication list was also provided. }\end{array}$ & $\begin{array}{l}\text { No pharmacist provided education } \\
\text { and follow-up. }\end{array}$ & $\begin{array}{l}\text {-All-cause readmission at } 30 \text { days } \\
\text {-Medication adherence and } \\
\text { literary scores at } 30 \text { days post- } \\
\text { discharge }^{\mathrm{a}}\end{array}$ \\
\hline
\end{tabular}

(Continued) 
Table I (Continued).

\begin{tabular}{|c|c|c|c|c|}
\hline $\begin{array}{l}\text { Study, Year, } \\
\text { Country } \\
\text { Design }\end{array}$ & $\mathbf{N}$ and Study Population & Intervention(s) & Comparator & Outcome \\
\hline $\begin{array}{l}\text { Cabilan et al, } \\
2019^{38} \\
\text { Australia } \\
\text { Quasi- } \\
\text { randomized } \\
\text { trial }\end{array}$ & $\begin{array}{l}5 \mathrm{I} \text { adults discharged from short } \\
\text { stay unit of emergency department } \\
\text { with a prescription for } \\
\text { medications that they have not } \\
\text { been prescribed before or in the } \\
\text { last } 12 \text { months }\end{array}$ & $\begin{array}{l}\text { Pharmacist provided bed-side } \\
\text { discharge counselling. } \\
\text { Comprehension was assessed } \\
\text { using teach-back approach. } \\
\text { Prescription, medications, and } \\
\text { written information were also } \\
\text { provided. }\end{array}$ & $\begin{array}{l}\text { Doctors provided prescription and } \\
\text { medication counselling. }\end{array}$ & $\begin{array}{l}\text {-Patient satisfaction with } \\
\text { information about medications }{ }^{\mathrm{a}} \\
\text {-Number of re-presentations to } \\
\text { emergency department }\end{array}$ \\
\hline $\begin{array}{l}\text { Chakravarthy } \\
\text { et al, } 2018^{39} \\
\text { United States } \\
\text { Randomized } \\
\text { controlled } \\
\text { trial }\end{array}$ & $\begin{array}{l}52 \text { adults receiving an outpatient } \\
\text { prescription for opioid analgesic }\end{array}$ & $\begin{array}{l}\text { In addition to standard care, } \\
\text { participants watched a 6-minute } \\
\text { video on proper opioid use prior } \\
\text { to discharge. }\end{array}$ & $\begin{array}{l}\text { Standard care in which nurse } \\
\text { provided verbal discharge } \\
\text { instructions and information sheet } \\
\text { on opioids (eg, side effects, drug } \\
\text { interactions, precautions). }\end{array}$ & $\begin{array}{l}\text {-Patient knowledge acquisition } \\
\text { regarding the opioid use, risks, and } \\
\text { disposal }^{\mathrm{a}}\end{array}$ \\
\hline $\begin{array}{l}\text { Chan et al, } \\
2018^{40} \\
\text { Australia } \\
\text { Non- } \\
\text { randomized } \\
\text { trial }\end{array}$ & $\begin{array}{l}233 \text { patients discharged with } \\
\text { oxycodone for acute pain }\end{array}$ & $\begin{array}{l}\text { Doctors provided education on } \\
\text { prescribed opioid and acute pain } \\
\text { management. Pain management } \\
\text { factsheets were distributed. }\end{array}$ & Usual care (not specified) & $\begin{array}{l}\text {-Patient knowledge on } \\
\text { recommended dose }^{\mathrm{a}} \text { and adverse } \\
\text { effects }^{\mathrm{a}} \\
\text {-Patient self-reported practice } \\
\text { after discharge } \\
\text {-Factsheet distribution }\end{array}$ \\
\hline $\begin{array}{l}\text { Chedepudi } \\
\text { et al, 2017 } 7^{41} \\
\text { India } \\
\text { Before-and- } \\
\text { after study }\end{array}$ & $\begin{array}{l}70 \text { adults on oral anticoagulant } \\
\text { therapy (acenocoumarol) }\end{array}$ & $\begin{array}{l}\text { Clinical pharmacist provided } \\
\text { educational sessions and leaflets } \\
\text { with information on } \\
\text { acenocoumarol therapy. }\end{array}$ & $\begin{array}{l}\text { Participants before they received } \\
\text { patient education sessions and } \\
\text { education leaflets. }\end{array}$ & $\begin{array}{l}\text {-Knowledge on anticoagulation } \\
\text { therapy }^{\mathrm{a}}\end{array}$ \\
\hline $\begin{array}{l}\text { Christy et al, } \\
2016^{42} \\
\text { United States } \\
\text { Cohort Study }\end{array}$ & $\begin{array}{l}795 \text { adults discharged from general } \\
\text { medical unit }\end{array}$ & $\begin{array}{l}\text { At discharge, pharmacy resident } \\
\text { or student performed medication } \\
\text { review and beside counselling. } \\
\text { Discharge medications and } \\
\text { information pamphlets are } \\
\text { distributed at bedside. Patients } \\
\text { were referred to ambulatory } \\
\text { clinics and received follow-up calls. }\end{array}$ & Usual hospital discharge service & $\begin{array}{l}\text {-30-day all-cause readmissions } \\
\text {-Hospital admissions from } \\
\text { emergency department visits } \\
\text {-Medication satisfaction with } \\
\text { medication education and timely } \\
\text { bedside medication delivery }\end{array}$ \\
\hline $\begin{array}{l}\text { Cordasco } \\
\text { et al, } 2009^{43} \\
\text { United States } \\
\text { Randomized } \\
\text { controlled } \\
\text { trial }\end{array}$ & $\begin{array}{l}210 \text { adults with congestive heart } \\
\text { failure or coronary artery disease } \\
\text { prescribed } 3 \text { or more medications } \\
\text { at discharge }\end{array}$ & $\begin{array}{l}\text { Provision of paper-based low- } \\
\text { literacy medication tool in addition } \\
\text { to standard care. }\end{array}$ & $\begin{array}{l}\text { Standard care with provision of } \\
\text { 30-day medication supply, } \\
\text { discharge education provided by } \\
\text { unit nurse, written instructions } \\
\text { from discharge physician and on } \\
\text { pill bottle labels. }\end{array}$ & $\begin{array}{l}\text {-Medication knowledge } \\
\text {-Self-reported medication } \\
\text { adherence } \\
\text {-Self-reported pill count } \\
\text {-Nurse rated tool acceptability } \\
\text {-Self-reported missed dose (higher } \\
\text { in intervention arm) }\end{array}$ \\
\hline $\begin{array}{l}\text { Cote et al, } \\
2015^{44} \\
\text { Canada } \\
\text { Quasi- } \\
\text { randomized } \\
\text { trial }\end{array}$ & $\begin{array}{l}\text { I } 79 \text { adults with HIV on anti- } \\
\text { retroviral therapy for at least } 6 \\
\text { months }\end{array}$ & $\begin{array}{l}\text { Virtual follow-up with virtual } \\
\text { nurse in addition to traditional } \\
\text { follow-up. }\end{array}$ & $\begin{array}{l}\text { Traditional follow-up meetings } \\
\text { with health care professionals over } \\
3 \text { to } 4 \text { months. Meetings consist of } \\
\text { discussions on medications, } \\
\text { symptoms, and encountered } \\
\text { problems. }\end{array}$ & $\begin{array}{l}\text {-Self-efficacy } \\
\text {-Medication adherence }\end{array}$ \\
\hline
\end{tabular}

(Continued) 
Table I (Continued).

\begin{tabular}{|c|c|c|c|c|}
\hline $\begin{array}{l}\text { Study, Year, } \\
\text { Country } \\
\text { Design }\end{array}$ & $\mathbf{N}$ and Study Population & Intervention(s) & Comparator & Outcome \\
\hline $\begin{array}{l}\text { Davis et al, } \\
2012^{45} \\
\text { United States } \\
\text { Randomized } \\
\text { controlled } \\
\text { trial }\end{array}$ & $\begin{array}{l}\text { I } 25 \text { adults with systolic or } \\
\text { diastolic heart failure and mild } \\
\text { cognitive impairment }\end{array}$ & $\begin{array}{l}\text { Heart failure case manager } \\
\text { delivered customized education on } \\
\text { heart failure self-care and } \\
\text { problem-solving during } \\
\text { hospitalization. Supplies such as } \\
\text { workbook and audiotape were } \\
\text { provided provided to help patients } \\
\text { organize, document, or remember } \\
\text { information. Follow-up was also } \\
\text { conducted. }\end{array}$ & $\begin{array}{l}\text { Heart failure case manager } \\
\text { provided standard heart failure } \\
\text { discharge teaching with verbal } \\
\text { review of patient education } \\
\text { booklet on symptom management, } \\
\text { lifestyle modifications, and } \\
\text { medication adherence. }\end{array}$ & $\begin{array}{l}\text {-Mean knowledge score }{ }^{\mathrm{a}} \\
\text {-Self-care mean change score } \\
\text {-30-day readmission rate }\end{array}$ \\
\hline $\begin{array}{l}\text { De La Fuente } \\
\text { et al, } 2011^{46} \\
\text { Spain } \\
\text { Randomized } \\
\text { controlled } \\
\text { trial }\end{array}$ & $\begin{array}{l}59 \text { patients hospitalized for } 3 \\
\text { months or more and prescribed } 4 \\
\text { or more active ingredients at } \\
\text { discharge }\end{array}$ & $\begin{array}{l}\text { Pharmacist provided verbal and } \\
\text { written information regarding } \\
\text { their treatment at discharge. }\end{array}$ & $\begin{array}{l}\text { Did not receive verbal or written } \\
\text { pharmacotherapeutic information. }\end{array}$ & $\begin{array}{l}\text {-Adherence to treatment at } \\
\text { discharge } \\
\text {-Adherence to treatment in } \\
\text { telephone interview ( } 30-50 \text { days) } \\
\text { after discharge } \\
\text {-Hospital readmission rate } \\
\text {-Emergency department visits } \\
\text {-Mortality rate }\end{array}$ \\
\hline $\begin{array}{l}\text { Ducharme } \\
\text { et al, } 2011^{47} \\
\text { Canada } \\
\text { Randomized } \\
\text { controlled } \\
\text { trial }\end{array}$ & $\begin{array}{l}219 \text { children with asthma who } \\
\text { received albuterol and fluticasone } \\
\text { inhalers }\end{array}$ & $\begin{array}{l}\text { Treating emergency physician } \\
\text { recorded discharge and } \\
\text { management instructions on } \\
\text { written action plan for asthma } \\
\text { attacks coupled with a } \\
\text { prescription (WAP-P). WAP-P also } \\
\text { contained additional information } \\
\text { and tools for self-management. }\end{array}$ & $\begin{array}{l}\text { Treating emergency physician } \\
\text { recorded discharge and } \\
\text { management instructions on } \\
\text { standard unformatted } \\
\text { prescription. }\end{array}$ & $\begin{array}{l}\text {-Adherence to fluticasone } \\
\text {-Asthma control } \\
\text { a } \\
\text {-Percent of patients who filled oral } \\
\text { corticosteroid prescription } \\
\text {-Cessation of fluticasone }\end{array}$ \\
\hline $\begin{array}{l}\text { Feldman et al, } \\
2018^{48} \\
\text { United States } \\
\text { Non- } \\
\text { randomized } \\
\text { trial }\end{array}$ & $\begin{array}{l}985 \text { adult general medicine } \\
\text { inpatients }\end{array}$ & $\begin{array}{l}\text { Pharmacist conducted medication } \\
\text { review, provided education, and } \\
\text { identified and resolved } \\
\text { medication-related problems and } \\
\text { barriers. Follow-up was also } \\
\text { provided. Patients with higher } \\
\text { readmission risk worksheet score } \\
\text { received the service. }\end{array}$ & $\begin{array}{l}\text { Patients with lower readmission } \\
\text { risk worksheet score received } \\
\text { usual care (unspecified). }\end{array}$ & $\begin{array}{l}\text {-30-day readmissions }{ }^{\mathrm{a}} \\
-30 \text {-day readmissions in patients } \\
\text { with Medicare Fee for Service } \\
\text { insurance }\end{array}$ \\
\hline $\begin{array}{l}\text { Hyrkas et al, } \\
2014^{49} \\
\text { United States } \\
\text { Non- } \\
\text { randomized } \\
\text { trial }\end{array}$ & $\begin{array}{l}303 \text { adult patients on a medication } \\
\text { regimen discharged from inpatient } \\
\text { medical-surgical units }\end{array}$ & $\begin{array}{l}\text { Patients received either patient- } \\
\text { centered intervention or } \\
\text { motivational interviewing from } \\
\text { nurses. Both interventions were } \\
\text { targeted towards improving } \\
\text { medication adherence. }\end{array}$ & $\begin{array}{l}\text { Nurses performed medication } \\
\text { reconciliation and delivered } \\
\text { discharge instructions and } \\
\text { education. }\end{array}$ & $\begin{array}{l}\text {-Medication adherence } \\
\text {-Confidence in medication } \\
\text { adherence }^{\mathrm{b}} \\
\text {-Self-efficacy } \\
\text {-Therapeutic alliance } \\
\text {-Patient's experience } \\
\text {-Readmission rate } \\
\text {-Predictors of readmission }\end{array}$ \\
\hline
\end{tabular}

(Continued) 
Table I (Continued).

\begin{tabular}{|c|c|c|c|c|}
\hline $\begin{array}{l}\text { Study, Year, } \\
\text { Country } \\
\text { Design }\end{array}$ & $\mathbf{N}$ and Study Population & Intervention(s) & Comparator & Outcome \\
\hline $\begin{array}{l}\text { Jiang et al, } \\
2016^{50} \\
\text { China } \\
\text { Randomized } \\
\text { controlled } \\
\text { trial }\end{array}$ & $\begin{array}{l}\text { I } 82 \text { adults with type } 2 \text { diabetes } \\
\text { receiving insulin therapy }\end{array}$ & $\begin{array}{l}\text { In addition to routine education, } \\
\text { diabetes nurse specialist delivered } \\
\text { picture description education } \\
\text { seminars to provide knowledge on } \\
\text { insulin use, benefit, side effects, } \\
\text { storage, and treatment targets. } \\
\text { Colored picture on insulin use was } \\
\text { employed in the sessions. }\end{array}$ & $\begin{array}{l}\text { Routine health education seminars } \\
\text { organized by diabetes nurse } \\
\text { specialist on diabetes, diet, } \\
\text { exercise, drug therapy, } \\
\text { complication prevention, blood } \\
\text { glucose monitoring. Standard } \\
\text { insulin injection method was } \\
\text { demonstrated } 4 \text { times. }\end{array}$ & $\begin{array}{l}\text {-Patient knowledge on diabetes, } \\
\text { insulin use, injection, hypoglycemia } \\
\text { and complications }{ }^{\mathrm{a}} \\
\text {-Adherence to insulin therapy }{ }^{\mathrm{a}} \\
\text {-FPG, postprandial glucose level, } \\
\text { HbAIC control } \\
\text {-Obtainment of correct diabetic } \\
\text { medications, standardized } \\
\text { monitoring, and timely referrals } 3 \\
\text { months after discharge }\end{array}$ \\
\hline $\begin{array}{l}\text { Jones et al, } \\
2018^{51} \\
\text { USA } \\
\text { Non- } \\
\text { randomized } \\
\text { trial }\end{array}$ & $\begin{array}{l}68 \text { adults at high risk of } \\
\text { readmission based on Parkland } \\
\text { risk score }\end{array}$ & $\begin{array}{l}\text { Transition of care pharmacist } \\
\text { obtained medication history, } \\
\text { performed medication } \\
\text { reconciliation, and provided } \\
\text { medication counselling. Follow-up } \\
\text { phone call after discharge was } \\
\text { conducted. }\end{array}$ & $\begin{array}{l}\text { Medical team without transition of } \\
\text { care pharmacist provided usual } \\
\text { care. }\end{array}$ & $\begin{array}{l}\text {-Readmission rate } \\
\text {-Composite outcome of a } \\
\text { readmission or emergency } \\
\text { department visit } \\
\text {-Number of patients with case } \\
\text { management or social work note }\end{array}$ \\
\hline $\begin{array}{l}\text { Kaestli et al, } \\
2016^{52} \\
\text { Switzerland } \\
\text { Cohort study }\end{array}$ & $\begin{array}{l}\text { I } 25 \text { children discharged from } \\
\text { pediatric emergency department }\end{array}$ & $\begin{array}{l}\text { Pharmacist provided drug } \\
\text { information leaflets to parents. } \\
\text { Leaflets contain information on } \\
\text { drug administration, interactions, } \\
\text { storage etc. }\end{array}$ & $\begin{array}{l}\text { Physician provided prescription } \\
\text { and brief explanation to parents. }\end{array}$ & $\begin{array}{l}\text {-Parental knowledge (on dose, } \\
\text { frequency, etc.) } \\
\text {-Parental satisfaction on discharge } \\
\text { information }\end{array}$ \\
\hline $\begin{array}{l}\text { Kapoor et al, } \\
2019^{53} \\
\text { United States } \\
\text { Randomized } \\
\text { controlled } \\
\text { trial }\end{array}$ & $\begin{array}{l}\text { I62 adults with new episode of } \\
\text { venous thromboembolism and } \\
\text { prescribed warfarin, direct oral } \\
\text { anticoagulants, low-molecular } \\
\text { weight heparin or fondaparinux }\end{array}$ & $\begin{array}{l}\text { Pharmacist assessed patient's self- } \\
\text { management of medications, } \\
\text { identified and discussed } \\
\text { knowledge gaps on medications } \\
\text { and conditions, and provided } \\
\text { colored medication list during } \\
\text { home visit. Nurse delivered } \\
\text { follow-up to provide further } \\
\text { education and to update } \\
\text { medication list. }\end{array}$ & $\begin{array}{l}\text { Usual care in which clinical } \\
\text { pharmacist met with patient } \\
\text { discharged from hospital (but not } \\
\text { those discharged from emergency } \\
\text { department or outpatient } \\
\text { settings). No medication } \\
\text { simulations or illustrated } \\
\text { instructions were used. }\end{array}$ & $\begin{array}{l}\text {-Care transition quality } \\
\text {-Patient knowledge } \\
\text {-Adherence }\end{array}$ \\
\hline $\begin{array}{l}\text { Khonsari } \\
\text { et al, } 2014^{54} \\
\text { Malaysia } \\
\text { Randomized } \\
\text { controlled } \\
\text { trial }\end{array}$ & $\begin{array}{l}62 \text { patients with acute coronary } \\
\text { syndrome }\end{array}$ & $\begin{array}{l}\text { Patient received automated text } \\
\text { message reminders before every } \\
\text { cardiac medication intake to } \\
\text { optimize adherence. }\end{array}$ & $\begin{array}{l}\text { Usual post-discharge care with } \\
\text { cardiac rehabilitation and follow- } \\
\text { up with cardiologist. }\end{array}$ & $\begin{array}{l}\text {-Medication adherence } \\
\text {-Heart function status } \\
\text { a } \\
\text {-Acute coronary syndrome- } \\
\text { related hospital readmission rate } \\
\text {-Death rate }\end{array}$ \\
\hline $\begin{array}{l}\text { Lam et al, } \\
2011^{55} \\
\text { Australia } \\
\text { Before-and- } \\
\text { after study }\end{array}$ & $\begin{array}{l}24 \text { older adults taking } 3 \text { or more } \\
\text { long-term medications }\end{array}$ & $\begin{array}{l}\text { Self-administration of medications } \\
\text { programme delivered by nurses } \\
\text { and pharmacists to allow patients } \\
\text { to learn to self-medicate in a } \\
\text { supported manner with education } \\
\text { and supervision. }\end{array}$ & Before participation in program. & $\begin{array}{l}\text {-Patients' competence to manage } \\
\text { medication }^{\mathrm{a}} \\
\text {-Non-adherent behaviour } \\
\text {-Adherent behaviour }\end{array}$ \\
\hline
\end{tabular}

(Continued) 
Table I (Continued).

\begin{tabular}{|c|c|c|c|c|}
\hline $\begin{array}{l}\text { Study, Year, } \\
\text { Country } \\
\text { Design }\end{array}$ & $\mathbf{N}$ and Study Population & Intervention(s) & Comparator & Outcome \\
\hline $\begin{array}{l}\text { Leguelinel- } \\
\text { Blache et al, } \\
2015^{56} \\
\text { France } \\
\text { Non- } \\
\text { randomized } \\
\text { trial }\end{array}$ & $\begin{array}{l}\text { I93 adults admitted to infectious } \\
\text { and tropical diseases or general } \\
\text { medicine unit }\end{array}$ & $\begin{array}{l}\text { Initial counselling session, } \\
\text { medication reconciliation, } \\
\text { inpatient follow-up and discharge } \\
\text { counselling performed by clinical } \\
\text { pharmacist. }\end{array}$ & $\begin{array}{l}\text { Medication reconciliation and } \\
\text { inpatient follow-up performed by } \\
\text { clinical pharmacist. }\end{array}$ & $\begin{array}{l}\text {-Rate of unfilled new } \\
\text { prescriptions }^{\mathrm{a}} \\
\text {-Adherence rate } \\
\text {-Post-discharge healthcare } \\
\text { utilization (ie readmissions, } \\
\text { emergency department visits) }\end{array}$ \\
\hline $\begin{array}{l}\text { Li et al, } \\
2016^{57} \\
\text { United States } \\
\text { Non- } \\
\text { randomized } \\
\text { trial }\end{array}$ & $\begin{array}{l}190 \text { adults discharged from general } \\
\text { medicine unit }\end{array}$ & $\begin{array}{l}\text { Pharmacist created best possible } \\
\text { medication discharge list, delivered } \\
\text { discharge medications to bedside, } \\
\text { provided counselling, and } \\
\text { communicated with follow-up } \\
\text { providers on discharge } \\
\text { medications. }\end{array}$ & Not specified & -30-day readmission rates \\
\hline $\begin{array}{l}\text { Louis- } \\
\text { Simonet et al, } \\
2004^{58} \\
\text { Switzerland } \\
\text { Non- } \\
\text { randomized } \\
\text { trial }\end{array}$ & $\begin{array}{l}410 \text { patients discharged from } \\
\text { internal medicine services and } \\
\text { prescribed one or more } \\
\text { medications }\end{array}$ & $\begin{array}{l}\text { Physicians provided patient- } \\
\text { centered interview at discharge to } \\
\text { discuss each discharge medications } \\
\text { and to address any questions or } \\
\text { concerns. Standardized treatment } \\
\text { card containing information on } \\
\text { discharge medications was } \\
\text { provided. }\end{array}$ & $\begin{array}{l}\text { Usual procedure in which medical } \\
\text { residents provided information on } \\
\text { discharge medications as judged } \\
\text { appropriate. }\end{array}$ & $\begin{array}{l}\text {-Percentage of medications for } \\
\text { which patients correctly knew: I) } \\
\text { purpose }^{\text {a }} \text { 2) possible side effects }{ }^{\mathrm{a}} \text {; } \\
\text { 3) precautions to observe } \\
\text {-Number of medications } \\
\text { discontinued after discharge } \\
\text {-Patient assessment of } \\
\text { standardized treatment card } \\
\text { usefulness }\end{array}$ \\
\hline $\begin{array}{l}\text { Lu et al, } \\
2017^{59} \\
\text { United States } \\
\text { Cohort study }\end{array}$ & 277 patients with heart failure & $\begin{array}{l}\text { Heart Failure Post-Discharge } \\
\text { Management Clinic provided } \\
\text { counselling on disease state, } \\
\text { lifestyle modifications, and } \\
\text { medications. Patient medication } \\
\text { regimen was assessed and } \\
\text { modified as needed at each visit. }\end{array}$ & Usual care (not specified) & $\begin{array}{l}\text {-Adherence to angiotensin- } \\
\text { converting enzyme inhibitors }{ }^{\mathrm{a}} \text {, to } \\
\text { aldosterone antagonist }{ }^{\mathrm{a}} \text {, to twice- } \\
\text { daily beta blockers }{ }^{\mathrm{a}} \text {, to once-daily } \\
\text { beta blockers, to all beta blockers, } \\
\text { and to digoxin }\end{array}$ \\
\hline $\begin{array}{l}\text { Luder et al, } \\
2015^{60} \\
\text { United States } \\
\text { Non- } \\
\text { randomized } \\
\text { trial }\end{array}$ & $\begin{array}{l}90 \text { adults with congestive heart } \\
\text { failure, chronic obstructive } \\
\text { pulmonary disease, or pneumonia }\end{array}$ & $\begin{array}{l}\text { Pharmacist provided medication } \\
\text { therapy management service } \\
\text { (contents include medication } \\
\text { review patient education and } \\
\text { counselling) and written materials } \\
\text { to aid in self-management. Patients } \\
\text { could also receive Care Transition } \\
\text { Intervention. }\end{array}$ & $\begin{array}{l}\text { Usual care consists of either no } \\
\text { intervention or Care Transition } \\
\text { Intervention (nurse home visit and } \\
\text { follow-up weekly calls) }\end{array}$ & $\begin{array}{l}\text {-30-day readmissions rate }{ }^{a} \\
\text {-Patient satisfaction }\end{array}$ \\
\hline $\begin{array}{l}\text { Manning et al, } \\
2007^{61} \\
\text { United States } \\
\text { Randomized } \\
\text { controlled } \\
\text { trial }\end{array}$ & $\begin{array}{l}\text { I } 38 \text { adults with more than } 3 \\
\text { discharge medications }\end{array}$ & $\begin{array}{l}\text { Durable Display at Discharge (3D) } \\
\text { medication sheets which displayed } \\
\text { sample of discharge medications } \\
\text { and listed medication name, } \\
\text { indication, administration time and } \\
\text { amount, and additional comments } \\
\text { and prompts. }\end{array}$ & $\begin{array}{l}\text { Personalized Medication Discharge } \\
\text { Worksheet which listed } \\
\text { medications and administration } \\
\text { times }\end{array}$ & $\begin{array}{l}\text {-Patient knowledge of prescribed } \\
\text { medications }{ }^{\mathrm{a}} \\
\text {-Patient satisfaction } \\
\text {-Self-reported medication errors }\end{array}$ \\
\hline
\end{tabular}

(Continued) 
Table I (Continued).

\begin{tabular}{|c|c|c|c|c|}
\hline $\begin{array}{l}\text { Study, Year, } \\
\text { Country } \\
\text { Design }\end{array}$ & $\mathbf{N}$ and Study Population & Intervention(s) & Comparator & Outcome \\
\hline $\begin{array}{l}\text { Marusic et al, } \\
2018^{63} \\
\text { Croatia } \\
\text { Randomized } \\
\text { controlled } \\
\text { trial }\end{array}$ & I 25 adults with type 2 diabetes & $\begin{array}{l}\text { In addition to standard care, } \\
\text { before discharge, physician } \\
\text { provided education on discharge } \\
\text { prescriptions. Information on } \\
\text { indication, medication regimen, } \\
\text { side effects, and importance of } \\
\text { adherence were provided. }\end{array}$ & $\begin{array}{l}\text { Standard care involving } \\
\text { standardized diabetes education } \\
\text { (on disease, complications, } \\
\text { medications, lifestyle } \\
\text { modifications, and self-monitoring } \\
\text { of blood glucose) and discharge } \\
\text { letter (lists diagnosis, intervention, } \\
\text { medications). }\end{array}$ & $\begin{array}{l}\text {-Number of adherent patients }{ }^{\mathrm{a}} \\
\text {-Number of patients who } \\
\text { experienced adverse outcomes }\end{array}$ \\
\hline $\begin{array}{l}\text { Marusic et al, } \\
2013^{62} \\
\text { Croatia } \\
\text { Randomized } \\
\text { controlled } \\
\text { trial }\end{array}$ & $\begin{array}{l}160 \text { older adults with } 2 \text { or more } \\
\text { discharge medications for } \\
\text { treatment of chronic conditions }\end{array}$ & $\begin{array}{l}\text { In addition to standard care, } \\
\text { before discharge, physician } \\
\text { provided education on discharge } \\
\text { prescriptions. Information on } \\
\text { indication, medication regimen, } \\
\text { side effects, and importance of } \\
\text { adherence were provided. }\end{array}$ & $\begin{array}{l}\text { Standard care involving discharge } \\
\text { letter which lists diagnosis, } \\
\text { intervention, and medications. }\end{array}$ & $\begin{array}{l}\text {-Medication compliance }{ }^{\mathrm{a}} \\
\text {-Readmission rate } \\
\text {-Emergency department visits }\end{array}$ \\
\hline $\begin{array}{l}\text { McCarthy } \\
\text { et al, } 2015^{64} \\
\text { United States } \\
\text { Randomized } \\
\text { controlled } \\
\text { trial }\end{array}$ & $\begin{array}{l}210 \text { adults discharged on } \\
\text { hydrocodone-acetaminophen } \\
\text { combination oral opioid analgesics }\end{array}$ & $\begin{array}{l}\text { MedSheets containing information } \\
\text { on hydrocodone-acetaminophen } \\
\text { presented by research assistant. } \\
\text { Physician answered any questions } \\
\text { that arose. }\end{array}$ & Usual care (unspecified) & $\begin{array}{l}- \text { Knowledge of precautions } \\
\text {-Knowledge of side effects } \\
{ }^{\mathrm{a}} \\
\text {-Likelihood of driving within } 6 \\
\text { hours after hydrocodone use }{ }^{\mathrm{a}} \\
- \text { Knowledge on interaction } \\
\text { between alcohol and hydrocodone } \\
\text {-Knowledge on opioid addiction }\end{array}$ \\
\hline $\begin{array}{l}\text { Miller et al, } \\
2016^{65} \\
\text { United States } \\
\text { Case-control } \\
\text { study }\end{array}$ & $\begin{array}{l}314 \text { patients discharged on } 4 \text { or } \\
\text { more maintenance medications }\end{array}$ & $\begin{array}{l}\text { Pharmacist provided medication } \\
\text { therapy management services over } \\
\text { the phone. A follow-up phone call } \\
\text { was also conducted. }\end{array}$ & Usual care (unspecified) & $\begin{array}{l}\text {-30-day readmission rate } \\
\text {-Pharmacist interventions and } \\
\text { recommendations on medication- } \\
\text { related problems }\end{array}$ \\
\hline $\begin{array}{l}\text { Moye et al, } \\
2018^{66} \\
\text { United States } \\
\text { Cohort study }\end{array}$ & $\begin{array}{l}\text { I } 77 \text { older adults admitted due to } \\
\text { heart failure }\end{array}$ & $\begin{array}{l}\text { In addition to standard care, } \\
\text { pharmacy team provided } \\
\text { medication reconciliation, } \\
\text { discharge counselling, personalized } \\
\text { binder to for patients to } \\
\text { document weight changes and } \\
\text { dietary intakes, as well as follow- } \\
\text { up. }\end{array}$ & $\begin{array}{l}\text { Standard of care with heart failure } \\
\text { education delivered by case } \\
\text { managers, physicians, and nurses. } \\
\text { Nurses ensured patient can } \\
\text { monitor weight changes at home, } \\
\text { invited patients to educational } \\
\text { sessions, provided heart failure } \\
\text { education packets, and contacted } \\
\text { patients within } 72 \text { hours post- } \\
\text { discharge. Case managers } \\
\text { arranged appointment within } 7 \\
\text { days after discharge. }\end{array}$ & -30-day readmission rate ${ }^{a}$ \\
\hline $\begin{array}{l}\text { Murphy et al, } \\
2019^{67} \\
\text { United States } \\
\text { Cohort study }\end{array}$ & $\begin{array}{l}359 \text { adults admitted due to heart } \\
\text { failure exacerbation and } 25 \mathrm{I} \text { adults } \\
\text { admitted due to acute myocardial } \\
\text { infarction }\end{array}$ & $\begin{array}{l}\text { Multidisciplinary team consisting of } \\
\text { cardiologist, pharmacists, } \\
\text { pharmacy residents, nurses, and } \\
\text { dieticians provided education and } \\
\text { counselling on disease state, } \\
\text { medication, and diet. Follow-ups } \\
\text { were also conducted. }\end{array}$ & Usual care (not specified) & $\begin{array}{l}-30 \text {-day readmission rate } \\
-72 \text {-hour emergency department } \\
\text { visits } \\
\text {-Mortality rates }\end{array}$ \\
\hline
\end{tabular}

(Continued) 
Table I (Continued).

\begin{tabular}{|c|c|c|c|c|}
\hline $\begin{array}{l}\text { Study, Year, } \\
\text { Country } \\
\text { Design }\end{array}$ & $\mathrm{N}$ and Study Population & Intervention(s) & Comparator & Outcome \\
\hline $\begin{array}{l}\text { Oliveira-Filho } \\
\text { et al, } 2014^{68} \\
\text { Brazil } \\
\text { Randomized } \\
\text { controlled } \\
\text { trial }\end{array}$ & $\begin{array}{l}61 \text { patients with cardiovascular } \\
\text { disease on antihypertensive } \\
\text { medication }\end{array}$ & $\begin{array}{l}\text { Pharmacist conducted medication } \\
\text { review at discharge, provided } \\
\text { counselling on disease and therapy, } \\
\text { and provided drug treatment card } \\
\text { in the form of refrigerator magnet. }\end{array}$ & Usual care (unspecified) & $\begin{array}{l}\text {-Medication adherence }{ }^{\mathrm{a}} \\
\text {-Readmission rate } \\
\text {-Mortality rate }\end{array}$ \\
\hline $\begin{array}{l}\text { Olives et al, } \\
2016^{69} \\
\text { United States } \\
\text { Randomized } \\
\text { controlled } \\
\text { trial }\end{array}$ & $\begin{array}{l}252 \text { I patients with outpatient } \\
\text { antibiotic prescription }\end{array}$ & $\begin{array}{l}\text { In addition to standard of care, } \\
\text { patients received either text } \\
\text { message or voice mail on } \\
\text { antibiotic self-administration. }\end{array}$ & $\begin{array}{l}\text { Standard of care discharge } \\
\text { instructions involving routine } \\
\text { verbal discharge instruction and } \\
\text { printed after-visit summary. }\end{array}$ & $\begin{array}{l}\text {-Discharge instruction modality } \\
\text { preference }^{\mathrm{b}} \\
-72 \text {-hour antibiotic retrieval } \\
\text {-Antibiotic course completion }\end{array}$ \\
\hline $\begin{array}{l}\text { Phatak et al, } \\
2016^{70} \\
\text { United States } \\
\text { Randomized } \\
\text { controlled } \\
\text { trial }\end{array}$ & $\begin{array}{l}278 \text { discharged on more than } 3 \\
\text { prescription medications or at } \\
\text { least I high-risk medication }\end{array}$ & $\begin{array}{l}\text { Pharmacist provided face-to-face } \\
\text { medication reconciliation, } \\
\text { personalized medication plan, and } \\
\text { discharge counselling. Pharmacist } \\
\text { conducted post-discharge follow- } \\
\text { up at day } 3,14 \text {, and } 30 \text {. }\end{array}$ & $\begin{array}{l}\text { Standard of care involving } \\
\text { medication reconciliation (without } \\
\text { face-to-face interaction) and daily } \\
\text { pharmacotherapy assessment by } \\
\text { clinical pharmacist, and discharge } \\
\text { counselling by physician or nurse. } \\
\text { Pharmacist conducted post- } \\
\text { discharge follow-up at day } 30 \text {. }\end{array}$ & $\begin{array}{l}\text {-30-day post-discharge } \\
\text { readmission or emergency } \\
\text { department visits } \\
\text {-Rate of adverse drug events or } \\
\text { medication errors } \\
\text {-Hospital Consumer Assessment } \\
\text { of Healthcare Providers and } \\
\text { Systems score in medication } \\
\text { knowledge domain }\end{array}$ \\
\hline $\begin{array}{l}\text { Press et al, } \\
2016^{71} \\
\text { United States } \\
\text { Randomized } \\
\text { controlled } \\
\text { trial }\end{array}$ & $\begin{array}{l}\text { I20 adults with asthma or COPD } \\
\text { who were discharged on } \\
\text { pressurized metered-dose inhaler }\end{array}$ & $\begin{array}{l}\text { Research educators provided } \\
\text { teach-to-goal intervention with } \\
\text { repeated demonstrations and } \\
\text { evaluations of participant inhaler } \\
\text { technique. Participants received } \\
\text { written instructions on inhaler } \\
\text { technique, and pamphlet on their } \\
\text { condition. }\end{array}$ & $\begin{array}{l}\text { Research educators provided brief } \\
\text { instruction intervention with } \\
\text { verbal (without demonstration) } \\
\text { and written instructions on inhaler } \\
\text { technique, verbal education on } \\
\text { condition, and assessment of } \\
\text { inhaler technique }\end{array}$ & $\begin{array}{l}\text {-Metered-dose inhaler misuse } \\
\text { immediately after discharge }{ }^{\mathrm{a}}, 30 \\
\text { days post-discharge, and } 90 \text { days } \\
\text { post-discharge } \\
\text {-Diskus misuse immediately after } \\
\text { discharge }{ }^{\mathrm{a}}, 30 \text { days post-discharge, } \\
\text { and } 90 \text { days post-discharge } \\
- \text {-Acute care events at } 30 \text { days }^{\mathrm{a}} \text { and } \\
90 \text { days } \\
\text {-Rescue metered dose inhaler use } \\
\text { or Diskus adherence at } 30 \text { and } 90 \\
\text { days }\end{array}$ \\
\hline $\begin{array}{l}\text { Renaudin } \\
\text { et al, } 2017^{72} \\
\text { France } \\
\text { Randomized } \\
\text { controlled } \\
\text { trial }\end{array}$ & $\begin{array}{l}\text { I } 400 \text { children hospitalized in } \\
\text { pediatric care unit or older adults } \\
\text { hospitalized in post-emergency } \\
\text { care unit }\end{array}$ & $\begin{array}{l}\text { Pharmacist conducted medication } \\
\text { reconciliation and treatment } \\
\text { review at admission and discharge. } \\
\text { At discharge, pharmacist also } \\
\text { provided comprehensive } \\
\text { medication history and patient } \\
\text { counselling, and communicated } \\
\text { discharge letter to community } \\
\text { providers. }\end{array}$ & $\begin{array}{l}\text { Medical staff collected medication } \\
\text { information at admission and } \\
\text { provided prescription at discharge. } \\
\text { Pharmacy team only involved in } \\
\text { dispensing medications. }\end{array}$ & $\begin{array}{l}\text {-Results not available (trial } \\
\text { protocol) } \\
\text {-Outcome measures include: all- } \\
\text { cause hospital readmission, death, } \\
\text { and/or emergency department } \\
\text { visits } 30 \text { days post-discharge. }\end{array}$ \\
\hline $\begin{array}{l}\text { Salmany et al, } \\
2018^{73} \\
\text { Jordan } \\
\text { Randomized } \\
\text { controlled } \\
\text { trial }\end{array}$ & $\begin{array}{l}332 \text { adults discharged from } \\
\text { inpatient service }\end{array}$ & $\begin{array}{l}\text { Patients received telephone } \\
\text { follow-up from pharmacist within } \\
72 \text { hours of discharge. Questions } \\
\text { regarding medications were asked } \\
\text { during the phone call. }\end{array}$ & $\begin{array}{l}\text { Patients did not receive telephone } \\
\text { follow-up provided by pharmacist. }\end{array}$ & $\begin{array}{l}\text {-Patient satisfaction } \\
\text {-30-day post-discharge hospital } \\
\text { readmission and emergency } \\
\text { department visits }\end{array}$ \\
\hline
\end{tabular}


Table I (Continued).

\begin{tabular}{|c|c|c|c|c|}
\hline $\begin{array}{l}\text { Study, Year, } \\
\text { Country } \\
\text { Design }\end{array}$ & $\mathbf{N}$ and Study Population & Intervention(s) & Comparator & Outcome \\
\hline $\begin{array}{l}\text { Sanii et al, } \\
2016^{74} \\
\text { Iran } \\
\text { Randomized } \\
\text { controlled } \\
\text { trial }\end{array}$ & $\begin{array}{l}154 \text { adults discharged from } \\
\text { respiratory ward }\end{array}$ & $\begin{array}{l}\text { Pharmacist provided medication } \\
\text { reconciliation and education (on } \\
\text { inhaler technique, disease state, } \\
\text { and medications) at discharge. Two } \\
\text { follow-ups were provided at } 2 \\
\text { weeks and I month respectively. }\end{array}$ & $\begin{array}{l}\text { No pharmacist discharge } \\
\text { intervention. One follow-up I } \\
\text { month after discharge conducted } \\
\text { by pharmacist in which discharge } \\
\text { medications, inhaler technique, } \\
\text { and medication adherence were } \\
\text { assessed. }\end{array}$ & $\begin{array}{l}\text {-Treatment satisfaction }{ }^{\mathrm{a}} \\
\text {-Medication adherence } \\
\text {-Rate of medication-related } \\
\text { hospital readmission or emergency } \\
\text { department visit }\end{array}$ \\
\hline $\begin{array}{l}\text { Sarangarm } \\
\text { et al, 2012 }{ }^{75} \\
\text { United States } \\
\text { Non- } \\
\text { randomized } \\
\text { trial }\end{array}$ & $\begin{array}{l}279 \text { adults discharged from } \\
\text { internal medicine }\end{array}$ & $\begin{array}{l}\text { Pharmacist conducted medication } \\
\text { reconciliation and provided } \\
\text { discharge counselling on } \\
\text { medications and disease state. } \\
\text { Follow-up was conducted through } \\
\text { telephone calls. }\end{array}$ & $\begin{array}{l}\text { Usual care in which nurse } \\
\text { delivered prescription and } \\
\text { provided discharge counselling on } \\
\text { self-care management. No follow- } \\
\text { up or additional medication } \\
\text { reconciliation was provided. }\end{array}$ & $\begin{array}{l}\text {-Patient satisfaction } \\
\text {-Primary medication adherence } \\
\text {-30-day hospital reutilization } \\
\text { (combined readmissions and } \\
\text { emergency department visits) } \\
\text {-Predictors for hospital utilization } \\
\text {-Number of pharmacist } \\
\text { interventions }\end{array}$ \\
\hline $\begin{array}{l}\text { Schwalm et al, } \\
2015^{76} \\
\text { Canada } \\
\text { Randomized } \\
\text { controlled } \\
\text { trial }\end{array}$ & $\begin{array}{l}852 \text { adults with STEMI who } \\
\text { underwent coronary angiography } \\
\text { procedure }\end{array}$ & $\begin{array}{l}\text { Personalized letters detailing } \\
\text { medication information and } \\
\text { encouraging adherence was sent } \\
\text { to patient and family physician at I, } \\
5,8 \text {, and II months after } \\
\text { angiogram. }\end{array}$ & Usual care (not specified) & $\begin{array}{l}\text {-Portion of participants taking all } 4 \\
\text { cardiovascular medication classes } \\
\text { at } 12 \text { months } \\
\text {-Medication adherence }{ }^{\mathrm{a}} \\
\text {-Persistence to all } 5 \text { medications at } \\
3 \text { and } 12 \text { months } \\
\text {-Number of discussions with } \\
\text { health care providers on } \\
\text { medications }\end{array}$ \\
\hline $\begin{array}{l}\text { Send et al, } \\
2014^{77} \\
\text { Germany } \\
\text { Non- } \\
\text { randomized } \\
\text { trial }\end{array}$ & $\begin{array}{l}90 \text { patients who took one or more } \\
\text { drug }\end{array}$ & $\begin{array}{l}\text { Physicians provided enhanced } \\
\text { medication plan (generated in } \\
\text { semi-automatic fashion using } \\
\text { electronic database) containing } \\
\text { information on indication, drug } \\
\text { handling recommendations, and } \\
\text { administration instructions. }\end{array}$ & $\begin{array}{l}\text { Physicians provided simplified } \\
\text { medication plan (containing brand } \\
\text { name, drug regimen, and } \\
\text { physician's recommendations) and } \\
\text { discharge education. }\end{array}$ & $\begin{array}{l}\text {-Patient knowledge } \mathrm{e}^{\mathrm{a}} \\
\text {-Number of drugs mentioned and } \\
\text { explained } \\
\text {-Number of drug administration } \\
\text { recommendations given by } \\
\text { physician } \\
\text {-Time physician spent on patient } \\
\text { education }^{\mathrm{a}}\end{array}$ \\
\hline $\begin{array}{l}\text { Shaver et al, } \\
2019^{78} \\
\text { United States } \\
\text { Non- } \\
\text { randomized } \\
\text { trial }\end{array}$ & $\begin{array}{l}1219 \text { adults with cardiovascular } \\
\text { disease (eg acute myocardial } \\
\text { infarction, heart failure, atrial } \\
\text { fibrillation/flutter, stroke, } \\
\text { pulmonary embolism, etc.) or } \\
\text { adults taking narrow therapeutic } \\
\text { index medications (eg } \\
\text { anticoagulants) }\end{array}$ & $\begin{array}{l}\text { Transition of Care program in } \\
\text { addition to Prescriptions Plus } \\
\text { Program. Patients received } \\
\text { telephone calls after discharge to } \\
\text { encourage adherence and } \\
\text { attendance at follow-up } \\
\text { appointments. Pharmacy student } \\
\text { made the phone calls with } \\
\text { pharmacist available to answer } \\
\text { patient questions if asked. }\end{array}$ & $\begin{array}{l}\text { Prescriptions Plus Program in } \\
\text { which patients were provided with } \\
\text { bedside discharge counselling and } \\
\text { 30-day medication supply before } \\
\text { discharge. No Transitions of Care } \\
\text { program }\end{array}$ & $\begin{array}{l}\text {-30-day all cause readmission }{ }^{\mathrm{a}} \\
-30-\text { day related admissions }\end{array}$ \\
\hline $\begin{array}{l}\text { Shull et al, } \\
2018^{79} \\
\text { United States } \\
\text { Cohort study }\end{array}$ & $\begin{array}{l}1059 \text { patients discharged from } \\
\text { hospital }\end{array}$ & $\begin{array}{l}\text { Pharmacist conducted medication } \\
\text { reconciliation, medication therapy } \\
\text { management, patient education } \\
\text { and assessed access to care at all } \\
\text { stages of hospitalization. Bridge } \\
\text { care coordinator conducted } \\
\text { follow-ups. }\end{array}$ & $\begin{array}{l}\text { Senior medical resident led } \\
\text { discharge process with standard } \\
\text { medication reconciliation } \\
\text { performed by medical team and } \\
\text { medication education performed } \\
\text { by nurse. The outpatient } \\
\text { pharmacy is offered to fill } \\
\text { prescriptions at discharge. }\end{array}$ & $\begin{array}{l}\text {-Readmission rate within } 30 \text { days }^{\mathrm{a}} \\
\text {-Estimated net benefit of the } \\
\text { transition-of-care program } \\
\text { through reduced unplanned } 30 \text {-day } \\
\text { readmission }\end{array}$ \\
\hline
\end{tabular}

(Continued) 
Table I (Continued).

\begin{tabular}{|c|c|c|c|c|}
\hline $\begin{array}{l}\text { Study, Year, } \\
\text { Country } \\
\text { Design }\end{array}$ & $\mathbf{N}$ and Study Population & Intervention(s) & Comparator & Outcome \\
\hline $\begin{array}{l}\text { Singh et al, } \\
2018^{80} \\
\text { Canada } \\
\text { Randomized } \\
\text { controlled } \\
\text { trial }\end{array}$ & $\begin{array}{l}80 \text { opioid naïve adults post foot } \\
\text { and ankle surgery }\end{array}$ & $\begin{array}{l}\text { Participants received written } \\
\text { discharge instructions on } \\
\text { acetaminophen, ibuprofen, and } \\
\text { opioid use in addition to usual } \\
\text { care. }\end{array}$ & $\begin{array}{l}\text { Usual care with just a prescription. } \\
\text { No written discharge instructions. }\end{array}$ & $\begin{array}{l}\text {-Postoperative pain satisfaction } \\
\text {-Modified brief pain inventory } \\
\text { score } \\
\text {-Interference scores } \\
\text {-Total prescriptions used } \\
\text {-Renewal of prescription } \\
\text { medication } \\
\text {-Number of participants who } \\
\text { returned unused medication to } \\
\text { pharmacy }\end{array}$ \\
\hline $\begin{array}{l}\text { Sinha et al, } \\
2019^{81} \\
\text { United States } \\
\text { Before-and- } \\
\text { after study }\end{array}$ & $\begin{array}{l}40 \text { adults discharged from general } \\
\text { medicine service }\end{array}$ & $\begin{array}{l}\text { Participants viewed videos that } \\
\text { target barriers for successful } \\
\text { transition from hospital to home. }\end{array}$ & $\begin{array}{l}\text { Participants before } \\
\text { implementation of intervention } \\
\text { (video). }\end{array}$ & $\begin{array}{l}\text {-Median self-efficacy score }{ }^{a} \\
\text {-Percent of participants who found } \\
\text { the intervention to be helpful } \\
\text {-Percent of participants that } \\
\text { correctly answered at least } 4 / 5 \text { of } \\
\text { knowledge assessment questions }\end{array}$ \\
\hline $\begin{array}{l}\text { Smith et al, } \\
2017^{82} \\
\text { United States } \\
\text { Before-and- } \\
\text { after }\end{array}$ & $\begin{array}{l}265 \text { patients with acute myocardial } \\
\text { infraction }\end{array}$ & $\begin{array}{l}\text { Patients received Heart Attack } \\
\text { Program Guide and education } \\
\text { during hospitalization, and follow- } \\
\text { up and cardiac rehabilitation after } \\
\text { discharge. }\end{array}$ & Unspecified & -30-day readmission rates \\
\hline $\begin{array}{l}\text { Tuttle et al, } \\
2018^{83} \\
\text { United States } \\
\text { Randomized } \\
\text { controlled } \\
\text { trial }\end{array}$ & $\begin{array}{l}141 \text { adults with chronic kidney } \\
\text { disease stages } 3-5 \text { not treated by } \\
\text { dialysis hospitalized for acute } \\
\text { illness }\end{array}$ & $\begin{array}{l}\text { In addition to usual care, } \\
\text { pharmacist provided home visits } \\
\text { after discharge to perform } \\
\text { medication review, to create } \\
\text { medication action plan, and to } \\
\text { deliver medication list and } \\
\text { counselling. }\end{array}$ & $\begin{array}{l}\text { Usual care in which, at discharge, } \\
\text { nurse provided electronic health } \\
\text { record-derived drug list and } \\
\text { discharge prescriptions. Nurse } \\
\text { also educated patients on disease } \\
\text { management, importance of } \\
\text { adherence and follow-up, and the } \\
\text { need to provide health care } \\
\text { providers with the discharge } \\
\text { medication list. }\end{array}$ & $\begin{array}{l}\text {-Acute care utilization } \\
\text { (readmission, emergency } \\
\text { department and urgent care visits) } \\
\text { within } 90 \text { days post-discharge } \\
\text {-Achievement of guideline-based } \\
\text { goals } \\
\text {-Rate of adverse events }\end{array}$ \\
\hline $\begin{array}{l}\text { Vuong et al, } \\
2008^{84} \\
\text { Australia } \\
\text { Randomized } \\
\text { controlled } \\
\text { trial }\end{array}$ & $\begin{array}{l}316 \text { older adults taking } 3 \text { or more } \\
\text { medications with dexterity, } \\
\text { language, hearing, or visual } \\
\text { difficulties }\end{array}$ & $\begin{array}{l}\text { In addition to standard care, } \\
\text { community liaison pharmacist } \\
\text { provided home visits following } \\
\text { discharge to assess patient's } \\
\text { knowledge, management, and } \\
\text { compliance with medication } \\
\text { regimen. }\end{array}$ & $\begin{array}{l}\text { Standard care in which pharmacist } \\
\text { provided discharge counselling and } \\
\text { compliance aids, and } \\
\text { communicated with primary } \\
\text { providers when needed. }\end{array}$ & $\begin{array}{l}\text {-Self-perceived medication } \\
\text { understanding } \\
\text {-Mean overall medication } \\
\text { knowledge score }^{\mathrm{a}} \\
\text {-Medication adherence }\end{array}$ \\
\hline $\begin{array}{l}\text { Walker et al, } \\
2009^{85} \\
\text { United States } \\
\text { Quasi- } \\
\text { randomized } \\
\text { trial }\end{array}$ & $\begin{array}{l}724 \text { adults at high risk of } \\
\text { medication related adverse events } \\
\text { post discharge }\end{array}$ & $\begin{array}{l}\text { Pharmacist conducted medication } \\
\text { reconciliation, discharge } \\
\text { counselling (including written } \\
\text { information to patient), } \\
\text { communicated discharge } \\
\text { medication list to follow-up care } \\
\text { provider, and did } 72 \mathrm{~h} \text { and } 30 \text {-day } \\
\text { follow-up phone call with patient. }\end{array}$ & $\begin{array}{l}\text { Nurse provided medication list } \\
\text { and discharge instructions. } \\
\text { Medicare beneficiaries received } \\
\text { follow-up phone call from nurse } \\
\text { within } 72 \text { hours after discharge to } \\
\text { identify and resolve any post- } \\
\text { discharge problems. No } \\
\text { pharmacist involved. }\end{array}$ & $\begin{array}{l}\text {-Medication discrepancies at } \\
\text { discharge }^{\mathrm{a}} \\
\text {-Readmission rate at } 14 \text { days }^{\mathrm{b}} \text { and } \\
30 \text { days post discharge } \\
\text {-Emergency department visits } \\
\text { within } 72 \text { hours of discharge }\end{array}$ \\
\hline
\end{tabular}

(Continued) 
Table I (Continued).

\begin{tabular}{|c|c|c|c|c|}
\hline $\begin{array}{l}\text { Study, Year, } \\
\text { Country } \\
\text { Design }\end{array}$ & $\mathrm{N}$ and Study Population & Intervention(s) & Comparator & Outcome \\
\hline $\begin{array}{l}\text { Zerafa et al, } \\
2011^{86} \\
\text { Malta } \\
\text { Randomized } \\
\text { controlled } \\
\text { trial }\end{array}$ & 80 adults post cardiac surgery & $\begin{array}{l}\text { Pharmacist provides medication } \\
\text { counselling and non- } \\
\text { pharmacological recommendations } \\
\text { for exercise and lifestyle. } \\
\text { Discharge medication chart } \\
\text { provided to aid in education. }\end{array}$ & $\begin{array}{l}\text { Doctor provided verbal and } \\
\text { written advice on medication } \\
\text { regiment. Physiotherapy team and } \\
\text { nurse provided education on } \\
\text { exercise regiments and lifestyle } \\
\text { changes respectively }\end{array}$ & -Mean percentage compliance \\
\hline
\end{tabular}

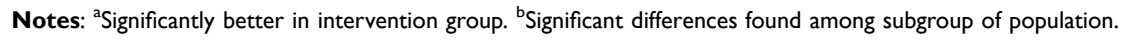

UCD-11 criteria (Table 3). ${ }^{47}$ Twenty percent $(n=12 / 60)$ of articles met more than 3 of the 11 UCD-11 criteria. ${ }^{29,33-}$ $36,43,47,52,64,76,81,86$ Of the 15 studies which discussed intervention development, 1 study reported consultation of expert opinion in the development process $(n=1 / 15),{ }^{34}$ and 3 studies reported usage of three or more iterative cycles in which outside opinions were consulted and prototype revisions were made accordingly $(\mathrm{n}=3 / 15) .{ }^{36,47,81}$ In contrast to these under-reported criteria, end user involvement in evaluation of the intervention, part of our study eligibility criteria, was consistently reported by all 15 studies $(n=15 / 15)$ that explicitly discussed intervention development. ${ }^{29,31,33-}$ $36,43,47,51,52,64,76,81,82,86$

\section{Content}

\section{Physical/Electronic Medication List Interventions}

For details regarding interventions including physical or electronic medication lists, see "Table 4". These included any interventions that contained the patient's medication lists in either a physical (eg paper) or electronic format. While $63.3 \%$ ( $\mathrm{n}=$ 38/60) interventions included a physical or electronic document containing information regarding the patient's medications (eg customized medication list, medication information pamphlet on a particular medication), ${ }^{28,29,33-43,45-47,50,52,53,55,56,58-}$ $68,71,76,77,83,85,86$ only $36.7 \% \quad(\mathrm{n}=22 / 60)$ interventions included a list of the patient's medications. ${ }^{28,29,33-}$ $37,43,45,53,55,56,58,60,61,65-67,77,83,85,86$ Of the 38 interventions with a physical or electronic document pertaining to the patient's medications, the content related to medication information included varied. Many included an indication/reason for use $(52.6 \%, \mathrm{n}=20 / 38),{ }^{29,33-37,40,43,47,52,53,56,58,61-64,66,76,77}$ timing of administration $(42.1 \%, \mathrm{n}=16 / 38),{ }^{34-37,40,43,52,53,55,60-}$ $64,77,86$ frequency of administration $(42.1 \%, \mathrm{n}=16 / 38),{ }^{34,36,37,40,43,47,52,53,56,58,60,61,64,66,77,86}$ and side effects $(34.2 \%, \mathrm{n}=$ 13/38). ${ }^{34,36,39,40,50,52,56,58,61-64,66}$ Ten percent $(n=6 / 60)$ of interventions included an electronic component. ${ }^{29,39,44,54,69,81}$

\section{Design}

\section{Mode of Delivery}

Interventions included in the study were classified based on their mode of delivery into either physical, verbal, electronic or a combination of these modalities. The majority of interventions consisted of multiple components $(78.3 \%, n=47)^{27-38,41-43,45-}$ 51,53,55-60,63-72,74,75,78,79,82,83,85,86 with 41.7\% $(\mathrm{n}=25)$ of interventions including a follow-up component.$27,30,33,34,36,37,42,44,45,48,51,53,59,65-67,70,73-75,79,82-85$ Sixty-percent $(n=37 / 60)$ of interventions, used physical and verbal means to deliver various components (eg medication list and patient education). ${ }^{28,29,33-43,45,46,49,50,53,55,56,58-60,62-69,71,74,82,83,85,86}$ Three of these interventions also included an electronically delivered component. ${ }^{29,39,69}$ Some interventions had only one mode of delivery: thirty percent $(n=13 / 60)$ of interventions were only verbally communicated to the patient, ${ }^{30-32,48,51,57,70,72,73,75,78,79,84}$ while $10 \%(n=6 / 60)$ were solely physically provided to the patient ${ }^{47,52,61,76,77,80}$ and $5 \%(n=3 / 60)$ were only electronically delivered. ${ }^{44,54,81}$ A small number of interventions did not involve direct delivery to patients. One of the interventions consisted of a letter mailed to the patient ${ }^{76}$ and family physician while another intervention involved a referral to outpatient providers if a need was identified. $^{27}$ 


\begin{tabular}{|c|c|c|c|c|c|c|c|}
\hline \multirow{2}{*}{$\begin{array}{l}\text { Study } \\
\text { (Author, } \\
\text { Year) }\end{array}$} & \multirow[t]{2}{*}{ Intervention Delivered by } & \multicolumn{2}{|c|}{$\begin{array}{l}\text { Timepoint During } \\
\text { Discharge }\end{array}$} & \multirow{2}{*}{$\begin{array}{c}\text { Mode of } \\
\text { Delivery to } \\
\text { Patient or } \\
\text { Caregiver* }\end{array}$} & \multirow[t]{2}{*}{$\begin{array}{l}\text { Components of } \\
\text { Intervention }\end{array}$} & \multirow[t]{2}{*}{ Content of Intervention Tool } & \multirow[t]{2}{*}{$\begin{array}{c}\text { Personalized } \\
\text { to Patients }\end{array}$} \\
\hline & & Before & After & & & & \\
\hline $\begin{array}{l}\text { Acomb et al } \\
2013^{27}\end{array}$ & $\begin{array}{c}\text { Pharmacists, Pharmacy technicians, } \\
\text { Primary care providers (numbers } \\
\text { unspecified) }\end{array}$ & & $x$ & Other & $\begin{array}{l}\text { Medicines Care Plan } \\
\text { Follow-up }\end{array}$ & $\begin{array}{l}\text { Medicines Care Plan: added to patient's discharge } \\
\text { communication if patient was determined to have } \\
\text { medicines related needs post-discharge by pharmacists and } \\
\text { pharmacy technicians. } \\
\text { Follow-up: Patients in hospital were referred to primary } \\
\text { care providers outside the hospital who would provide } \\
\text { follow-up care if a need was identified. Primary care } \\
\text { providers include general physicians, community } \\
\text { pharmacists, district nurses etc.). }\end{array}$ & $x$ \\
\hline $\begin{array}{l}\text { Al-Hashar } \\
\text { et al } 2018^{28}\end{array}$ & I Pharmacist & $x$ & & $\mathrm{P}, \mathrm{V}$ & $\begin{array}{l}\text { Pharmacist service on } \\
\text { admission and } \\
\text { discharge } \\
\text { Medication list }\end{array}$ & $\begin{array}{l}\text { On admission: medication history, identification of } \\
\text { counselling needs, reconciliation, and identification of } \\
\text { unintentional medication discrepancies } \\
\text { On discharge: medication reconciliation and identification } \\
\text { of unintentional medication discrepancies. Dispensing } \\
\text { medications at bedside. Provision of counselling about the } \\
\text { medication and take-home medication list. }\end{array}$ & $x$ \\
\hline $\begin{array}{l}\text { Anderegg } \\
\text { et al } 2013^{29}\end{array}$ & I or 2 Pharmacy case managers & $x$ & & $P, V, E$ & $\begin{array}{l}\text { Pharmacist service on } \\
\text { admission, during } \\
\text { admission, and at } \\
\text { discharge } \\
\text { Medication list and } \\
\text { wallet card }\end{array}$ & $\begin{array}{l}\text { On admission: medication reconciliation, identification of } \\
\text { drug related problems } \\
\text { During admission: education every } 2 \text { or } 3 \text { days on drug } \\
\text { indications, goals of therapy, adverse drug events, drug } \\
\text { adherence mechanisms, and self-monitoring measures. } \\
\text { Pharmacotherapy assessment and recommendations to } \\
\text { inpatient physicians. } \\
\text { On or after discharge: provision of discharge education, } \\
\text { drug therapy list, and a wallet card detailing medication } \\
\text { name, indication, dosage, instructions; patients received in- } \\
\text { person education and counselling, or over the phone with } \\
\text { the paperwork mailed to them. }\end{array}$ & $x$ \\
\hline
\end{tabular}


Table 2 (Continued).

\begin{tabular}{|c|c|c|c|c|c|c|c|}
\hline \multirow{2}{*}{$\begin{array}{l}\text { Study } \\
\text { (Author, } \\
\text { Year) }\end{array}$} & \multirow[t]{2}{*}{ Intervention Delivered by } & \multicolumn{2}{|c|}{$\begin{array}{l}\text { Timepoint During } \\
\text { Discharge }\end{array}$} & \multirow{2}{*}{$\begin{array}{l}\text { Mode of } \\
\text { Delivery to } \\
\text { Patient or } \\
\text { Caregiver* }\end{array}$} & \multirow[t]{2}{*}{$\begin{array}{l}\text { Components of } \\
\text { Intervention }\end{array}$} & \multirow[t]{2}{*}{ Content of Intervention Tool } & \multirow[t]{2}{*}{$\begin{array}{c}\text { Personalized } \\
\text { to Patients }\end{array}$} \\
\hline & & Before & After & & & & \\
\hline $\begin{array}{l}\text { Bailey et al } \\
2019^{30}\end{array}$ & $\begin{array}{l}\text { Advanced practice nurse, } \\
\text { Registered nurse, Licensed practical } \\
\text { nurses, Pharmacists, Pharmacy } \\
\text { technicians, Social worker }\end{array}$ & $x$ & $\mathrm{x}$ & v & $\begin{array}{l}\text { Healthcare team } \\
\text { service before } \\
\text { discharge } \\
\text { Follow-up }\end{array}$ & $\begin{array}{l}\text { Before discharge: I) patient identification and enrollment } \\
\text { by lead nurses; 2) patient engagement by all members of } \\
\text { multidisciplinary team; 3) medication reconciliation and } \\
\text { medication therapy management led by pharmacists; 4) } \\
\text { discharge preparation involving planning, education, and } \\
\text { coordination of care. } \\
\text { After discharge: weekly telephone calls and biweekly home } \\
\text { visits by licensed practical nurse and pharmacy technician. } \\
\text { Social worker provided service if needed. }\end{array}$ & $x$ \\
\hline $\begin{array}{l}\text { Baky et al } \\
2018^{31}\end{array}$ & $\begin{array}{l}\text { I Administrative clerk, } \\
\text { I Pharmacist, } \\
\text { I Physician }\end{array}$ & $x$ & & v & $\begin{array}{l}\text { Follow-up } \\
\text { appointment } \\
\text { scheduling } \\
\text { Medication education } \\
\text { Early entry of } \\
\text { discharge order }\end{array}$ & $\begin{array}{l}\text { Appointment scheduling: administrative clerk attempted to } \\
\text { schedule appointments at I- or 2-weeks post-discharge for } \\
\text { patients with heart failure or acute coronary syndrome } \\
\text { respectively. } \\
\text { Medication education: pharmacist involved in morning } \\
\text { rounds provided medication education to patients. } \\
\text { Discharge orders: entered by physician the day before } \\
\text { patient discharge date to allow patients to receive } \\
\text { discharge instruction early next day, and to allow staff time } \\
\text { to schedule appointments and provide education. }\end{array}$ & \\
\hline $\begin{array}{l}\text { Balling et al } \\
2015^{32}\end{array}$ & $\begin{array}{c}\text { I Transition-of- } \\
\text { care pharmacist } \\
\text { Nurses, case managers, social } \\
\text { workers, and outpatient pharmacy } \\
\text { technicians assisted, when needed }\end{array}$ & $x$ & & v & $\begin{array}{l}\text { Pharmacist service on } \\
\text { admission and } \\
\text { discharge }\end{array}$ & $\begin{array}{l}\text { On admission: review of patient information (e.g. insurance } \\
\text { coverage, medication adherence, history and physical at } \\
\text { admission), coordination with outpatient pharmacy on } \\
\text { insurance and payment barriers. } \\
\text { On discharge: medication reconciliation and identification } \\
\text { of discrepancies. Medication and disease counselling. }\end{array}$ & $x$ \\
\hline
\end{tabular}


Table 2 (Continued)

\begin{tabular}{|c|c|c|c|c|c|c|c|}
\hline \multirow{2}{*}{$\begin{array}{l}\text { Study } \\
\text { (Author, } \\
\text { Year) }\end{array}$} & \multirow[t]{2}{*}{ Intervention Delivered by } & \multicolumn{2}{|c|}{$\begin{array}{l}\text { Timepoint During } \\
\text { Discharge }\end{array}$} & \multirow{2}{*}{$\begin{array}{l}\text { Mode of } \\
\text { Delivery to } \\
\text { Patient or } \\
\text { Caregiver* }\end{array}$} & \multirow[t]{2}{*}{$\begin{array}{l}\text { Components of } \\
\text { Intervention }\end{array}$} & \multirow[t]{2}{*}{ Content of Intervention Tool } & \multirow[t]{2}{*}{$\begin{array}{c}\text { Personalized } \\
\text { to Patients }\end{array}$} \\
\hline & & Before & After & & & & \\
\hline $\begin{array}{l}\text { Bonetti et al } \\
2018^{36}\end{array}$ & 2 Pharmacy residents & $\mathrm{x}$ & $\mathrm{x}$ & $\mathrm{P}, \mathrm{V}$ & $\begin{array}{l}\text { Pharmacy resident } \\
\text { service during } \\
\text { admission and at } \\
\text { discharge } \\
\text { Information leaflet } \\
\text { Follow-up }\end{array}$ & $\begin{array}{l}\text { During admission: Pharmaceutical interventions when } \\
\text { needed. } \\
\text { On discharge: Individual counselling sessions for patients/ } \\
\text { caregivers to assess patient's discharge medication and to } \\
\text { provide medication education. Provision of leaflet with all } \\
\text { the information from the sessions. } \\
\text { Follow-up: Reinforcement counselling over the phone } 3 \\
\text { and } 15 \text { days after discharge. }\end{array}$ & $x$ \\
\hline $\begin{array}{l}\text { Budiman et al } \\
2016^{37}\end{array}$ & I Pharmacist & $x$ & $x$ & $P, V$ & $\begin{array}{l}\text { Pharmacist service } \\
\text { Medication List } \\
\text { Follow-up }\end{array}$ & $\begin{array}{l}\text { Pharmacist service: medication reconciliation, assessment } \\
\text { of medication adherence and literacy, medication } \\
\text { education, counselling on lifestyle management, provision } \\
\text { of customized medication list (see Table } 3 \text { for details), and } \\
\text { coordination with discharge pharmacy to resolve insurance } \\
\text { barriers. } \\
\text { Follow-up: post-discharge phone call within } 48 \text { to } 72 \text { hours } \\
\text { and at } 30 \text { days. Assessment of side effects, adherence, and } \\
\text { medication knowledge. Any questions or concerns were } \\
\text { addressed. }\end{array}$ & $x$ \\
\hline $\begin{array}{l}\text { Cabilan et al } \\
2019^{38}\end{array}$ & I Pharmacist & $x$ & & $\mathrm{P}, \mathrm{V}$ & $\begin{array}{l}\text { Discharge medication } \\
\text { counselling } \\
\text { Consumer Medication } \\
\text { Information leaflet } \\
\text { Comprehension } \\
\text { assessment }\end{array}$ & $\begin{array}{l}\text { Counselling: involves assessment of patient's understanding } \\
\text { of the prescribed medication, explanation of medications, } \\
\text { and medication education (e.g. generic name, dose, reasons } \\
\text { for use, precautions, side-effects, drug/food interactions). } \\
\text { Consumer Medication Information leaflet: provided at start } \\
\text { of counselling. } \\
\text { Comprehension assessment: done through teach-back } \\
\text { approach. }\end{array}$ & $x$ \\
\hline $\begin{array}{l}\text { Chakravarthy } \\
\text { et al } 2018^{39}\end{array}$ & $\begin{array}{c}\text { I Nurse, } \\
\text { Research/Project team }\end{array}$ & $x$ & & $\mathrm{P}, \mathrm{V}, \mathrm{E}$ & $\begin{array}{l}\text { Informational video in } \\
\text { addition to standard } \\
\text { care }\end{array}$ & $\begin{array}{l}\text { Video: information on opioid safety, proper usage, storage, } \\
\text { and disposal. Video utilized whiteboard and markers to } \\
\text { illustrate contents with synchronized voiceover. }\end{array}$ & \\
\hline
\end{tabular}




\begin{tabular}{|c|c|c|c|c|c|c|c|}
\hline $\begin{array}{l}\text { Chan et al } \\
2018^{40}\end{array}$ & I Emergency physician & $x$ & & $P, V$ & Education & $\begin{array}{l}\text { Education: doctors used PAID mnemonic (precautions, } \\
\text { adverse effects, other interventions, dosage and duration } \\
\text { of opioid therapy) to educate patients on prescribed opioid } \\
\text { and acute pain management. Pain management factsheets } \\
\text { were distributed. }\end{array}$ & \\
\hline $\begin{array}{l}\text { Chedepu-di } \\
\text { et al } 2017^{41}\end{array}$ & $\begin{array}{l}\text { Clinical pharmacist } \\
\text { (number unspecified) }\end{array}$ & $x$ & & $P, V$ & $\begin{array}{l}\text { Education sessions } \\
\text { Education leaflets }\end{array}$ & $\begin{array}{l}\text { Education sessions: detailed verbal educational sessions } \\
\text { approximately } 30 \text {-minute-long on acenocoumarol therapy } \\
\text { (e.g., reason for use and side effects of medication, purpose } \\
\text { of INR, and missed dose instructions). } \\
\text { Educational leaflets: Details not specified. }\end{array}$ & \\
\hline $\begin{array}{l}\text { Christy et al } \\
2016^{42}\end{array}$ & $\begin{array}{l}\text { I pharmacy residents, or I } \\
\text { advanced pharmacy practice } \\
\text { experience student }\end{array}$ & $x$ & $x$ & $P, V$ & $\begin{array}{l}\text { Pharmacy service at } \\
\text { discharge } \\
\text { Patient information } \\
\text { pamphlet } \\
\text { Follow-up }\end{array}$ & $\begin{array}{l}\text { At discharge: review of discharge prescriptions to ensure } \\
\text { appropriateness. Beside delivery of discharge medications, } \\
\text { provision of medication education (i.e., drug name, dose, } \\
\text { indications, directions for use, side effects, precautions, } \\
\text { self-monitoring techniques, and missed dose instructions), } \\
\text { and distribution of patient information pamphlets. Patients } \\
\text { were referred to hospital-affiliated ambulatory clinics. } \\
\text { Follow-up: telephone calls } 7 \text { days post-discharge to } \\
\text { monitor efficacy and safety medication therapy as well as } \\
\text { patient health. }\end{array}$ & $x$ \\
\hline $\begin{array}{l}\text { Cordasco } \\
\text { et al } 2009^{43}\end{array}$ & I Nurse & $x$ & & $P, V$ & $\begin{array}{l}\text { Medication tool } \\
\text { Discharge education } \\
\text { and instructions }\end{array}$ & $\begin{array}{l}\text { Medication tool: paper-based, low-literacy tool that is color } \\
\text { coded and provides customized medication schedules with } \\
\text { pictures and icons. } \\
\text { Education and discharge instructions: education was } \\
\text { conducted using the medication tool. Discharge } \\
\text { instructions are provided as in standard care. }\end{array}$ & $\mathrm{x}$ \\
\hline
\end{tabular}

(Continued) 
Table 2 (Continued).

\begin{tabular}{|c|c|c|c|c|c|c|c|}
\hline \multirow{2}{*}{$\begin{array}{l}\text { Study } \\
\text { (Author, } \\
\text { Year) }\end{array}$} & \multirow[t]{2}{*}{ Intervention Delivered by } & \multicolumn{2}{|c|}{$\begin{array}{l}\text { Timepoint During } \\
\text { Discharge }\end{array}$} & \multirow{2}{*}{$\begin{array}{l}\text { Mode of } \\
\text { Delivery to } \\
\text { Patient or } \\
\text { Caregiver* }\end{array}$} & \multirow[t]{2}{*}{$\begin{array}{l}\text { Components of } \\
\text { Intervention }\end{array}$} & \multirow[t]{2}{*}{ Content of Intervention Tool } & \multirow[t]{2}{*}{$\begin{array}{c}\text { Personalized } \\
\text { to Patients }\end{array}$} \\
\hline & & Before & After & & & & \\
\hline $\begin{array}{l}\text { Cote et al } \\
2015^{44}\end{array}$ & I Nurse (virtual) & & $x$ & E & $\begin{array}{l}\text { Virtual follow-up in } \\
\text { addition to traditional } \\
\text { follow-up }\end{array}$ & $\begin{array}{l}\text { Virtual follow-up: consists of } 4 \text { sessions. In the sessions, } \\
\text { virtual nurse provided tailored teaching and shared } \\
\text { anecdotes of other individuals with HIV who coped } \\
\text { successfully } \\
\text { I }^{\text {st }} \text { session: development of self-assessment and } \\
\text { motivational skill. } \\
2^{\text {nd }} \text { session: emotional management and problem-solving } \\
\text { process for dealing with situations where medication intake } \\
\text { is awkward. } \\
3^{\text {rd }} \text { session: social relations and interaction with health } \\
\text { professionals. } \\
4^{\text {th }} \text { session: all the skills previously worked on are } \\
\text { consolidated. }\end{array}$ & $x$ \\
\hline $\begin{array}{l}\text { Davis et al } \\
2012^{45}\end{array}$ & $\begin{array}{l}\text { Heart Failure Case Manager } \\
\quad \text { (unclear profession) }\end{array}$ & $x$ & $x$ & $\mathrm{P}, \mathrm{V}$ & $\begin{array}{l}\text { Service during } \\
\text { hospitalization } \\
\text { Workbook and other } \\
\text { supplies } \\
\text { Follow-up }\end{array}$ & $\begin{array}{l}\text { During hospitalization: customized education, using } \\
\text { pictograms and association techniques, to aid in self-care } \\
\text { schedule development. Individualized problem-solving } \\
\text { sessions with structure self-care scenarios. } \\
\text { Workbook and other supplies: Workbook allowed patients } \\
\text { to create personal self-care schedule and medication } \\
\text { schedule, to track appointments, and to record symptoms. } \\
\text { Other supplies include refrigerator card with self-care } \\
\text { reminders and audiotape of education sessions. Both } \\
\text { workbook and refrigerator card used pictograms. } \\
\text { Follow-up: phone call within I-3 days post-discharge. } \\
\text { Involves teach-back session focused on self-care or other } \\
\text { knowledge that was identified to be of concern, review use } \\
\text { of provided materials, medication reconciliation, and } \\
\text { clarification of questions on self-care. }\end{array}$ & $x$ \\
\hline $\begin{array}{l}\text { De La Fuente } \\
\text { et al } 2011^{46}\end{array}$ & I Pharmacist & $\mathrm{x}$ & & $\mathrm{P}, \mathrm{V}$ & $\begin{array}{l}\text { Verbal and written } \\
\text { information at } \\
\text { discharge }\end{array}$ & $\begin{array}{l}\text { At discharge: pharmacotherapeutic information was } \\
\text { provided both verbally and in written form. Details } \\
\text { unspecified. }\end{array}$ & $x$ \\
\hline
\end{tabular}




\begin{tabular}{|c|c|c|c|c|c|c|c|}
\hline $\begin{array}{l}\text { Ducharme } \\
\text { et al } 2011^{47}\end{array}$ & I Emergency physician & $x$ & & $P$ & $\begin{array}{l}\text { Written action plan for } \\
\text { asthma attacks coupled } \\
\text { with a prescription } \\
\text { (WAP-P) }\end{array}$ & $\begin{array}{l}\text { WAP-P: includes discharge instructions, template for } \\
\text { exacerbation and chronic management, emphasize asthma } \\
\text { chronicity, self-assessment tool for asthma control, } \\
\text { prescription, chart copy, and take-home plan. }\end{array}$ & $x$ \\
\hline $\begin{array}{l}\text { Feldman et al } \\
2018^{48}\end{array}$ & I Community pharmacist & $x$ & $x$ & $\mathrm{v}$ & $\begin{array}{l}\text { Pharmacist service } \\
\text { during admission } \\
\text { Follow-up }\end{array}$ & $\begin{array}{l}\text { During admission: initial visit within } 2 \text { days of admission } \\
\text { included program introduction, medication review, follow- } \\
\text { up on issues identified on admission through medication } \\
\text { reconciliation, identify and resolve other medication } \\
\text { problems/barriers, patient education on medication. } \\
\text { Option to have newly prescribed medications delivered to } \\
\text { hospital room was offered } \\
\text { Follow-up: telephone calls at } 8 \text { days and } 25 \text { days after } \\
\text { discharge on medication issues, side effects, refill needs, } \\
\text { etc. }\end{array}$ & $x$ \\
\hline $\begin{array}{l}\text { Hyrkas et al } \\
2014^{49}\end{array}$ & I Nurse & $x$ & & $P, V$ & $\begin{array}{l}\text { I) Patient-centered } \\
\text { intervention } \\
\text { 2) Motivational } \\
\text { interviewing }\end{array}$ & $\begin{array}{l}\text { Patients received one of two interventions: } \\
\text { Patient-centered intervention: Involved teach-back method } \\
\text { and tailored tools such as pill boxes and reminders to aid in } \\
\text { self-management of medication regimens and to improve } \\
\text { medication adherence. } \\
\text { Motivational interviewing: Used techniques such as open- } \\
\text { ended questions and asking permission to discuss patient } \\
\text { understanding and concerns, to provide education, and to } \\
\text { improve medication adherence. }\end{array}$ & $x$ \\
\hline $\begin{array}{l}\text { Jiang et al } \\
2016^{50}\end{array}$ & I Diabetes nurse specialist & $x$ & & $P, V$ & $\begin{array}{l}\text { Picture Description } \\
\text { Education with class } \\
\text { and tool in addition to } \\
\text { routine seminar }\end{array}$ & $\begin{array}{l}\text { Class: provided knowledge on insulin (e.g. use, benefit and } \\
\text { storage) hypoglycemia, and treatment targets (e.g. blood } \\
\text { glucose levels, hemoglobin } A_{1} C \text { levels). Patients were } \\
\text { encouraged to participate in in-class discussions. } \\
\text { Tool: colored picture on insulin use. }\end{array}$ & \\
\hline
\end{tabular}


Table 2 (Continued).

\begin{tabular}{|c|c|c|c|c|c|c|c|}
\hline \multirow{2}{*}{$\begin{array}{l}\text { Study } \\
\text { (Author, } \\
\text { Year) }\end{array}$} & \multirow[t]{2}{*}{ Intervention Delivered by } & \multicolumn{2}{|c|}{$\begin{array}{l}\text { Timepoint During } \\
\text { Discharge }\end{array}$} & \multirow{2}{*}{$\begin{array}{l}\text { Mode of } \\
\text { Delivery to } \\
\text { Patient or } \\
\text { Caregiver* }\end{array}$} & \multirow[t]{2}{*}{$\begin{array}{l}\text { Components of } \\
\text { Intervention }\end{array}$} & \multirow[t]{2}{*}{ Content of Intervention Tool } & \multirow[t]{2}{*}{$\begin{array}{c}\text { Personalized } \\
\text { to Patients }\end{array}$} \\
\hline & & Before & After & & & & \\
\hline $\begin{array}{l}\text { Jones et al } \\
2018^{51}\end{array}$ & I Transition of care pharmacist & $x$ & $x$ & v & $\begin{array}{l}\text { Pharmacist service on } \\
\text { admission and } \\
\text { discharge } \\
\text { Follow-up }\end{array}$ & $\begin{array}{l}\text { On admission: pharmacy-initiated medication history, } \\
\text { medication reconciliation } \\
\text { On discharge: medication reconciliation, medication } \\
\text { counselling. } \\
\text { Follow-up: telephone call within } 3 \text { days of discharge. } \\
\text { Involved medication reconciliation, review of indications, } \\
\text { assessment of barriers to filling or taking medications, and } \\
\text { assessment of medication-related problems (e.g., adverse } \\
\text { drug reaction, etc.). }\end{array}$ & $x$ \\
\hline $\begin{array}{l}\text { Kaestli et al } \\
2016^{52}\end{array}$ & I Pharmacist & $x$ & & $P$ & $\begin{array}{l}\text { Drug information } \\
\text { leaflets }\end{array}$ & $\begin{array}{l}\text { Drug information leaflet: designed for commonly } \\
\text { prescribed drugs. Used patient-friendly language. } \\
\text { Contained information on pediatric drug administration, } \\
\text { interaction, storage, discontinuation etc. }\end{array}$ & \\
\hline $\begin{array}{l}\text { Kapoor et al } \\
2019^{53}\end{array}$ & $\begin{array}{l}\text { I Clinical Pharmacist } \\
\text { I Nurse Practitioner }\end{array}$ & & $x$ & $\mathrm{P}, \mathrm{V}$ & $\begin{array}{l}\text { Home visit } \\
\text { Medication list } \\
\text { Follow-up } \\
\text { anticoagulation expert } \\
\text { consultation }\end{array}$ & $\begin{array}{l}\text { During home visit: pharmacist I) assessed patient's } \\
\text { medication self-management proficiency; 2) identified } \\
\text { knowledge gaps on medications and condition through } \\
\text { open-ended questions and provided education; 3) provided } \\
\text { illustrated medication list and instructions. } \\
\text { During follow-up: phone consultation in which nurse I) } \\
\text { asked the same open-ended questions presented in home } \\
\text { visit; 2) reviewed and updated medication list; 3) and } \\
\text { provided updated instructions to patients. }\end{array}$ & $x$ \\
\hline $\begin{array}{l}\text { Khonsari et al } \\
2014^{54}\end{array}$ & $\begin{array}{l}\text { Intervention did not require } \\
\text { delivery/administration by an } \\
\text { individual }\end{array}$ & & $x$ & $\mathrm{E}$ & $\begin{array}{l}\text { Automated web-based } \\
\text { system managing short } \\
\text { message service (SMS) } \\
\text { reminders }\end{array}$ & $\begin{array}{l}\text { SMS reminder: Provision of automated text message } \\
\text { reminders before every cardiac medication intake. } \\
\text { Information text message information include patient's } \\
\text { name, medication name and quantity, and time of } \\
\text { administration. }\end{array}$ & $x$ \\
\hline
\end{tabular}




\begin{tabular}{|c|c|c|c|c|c|c|}
\hline $\begin{array}{l}\text { Lam et al } \\
2011^{55}\end{array}$ & $\begin{array}{l}\text { I Nurse } \\
\text { I Pharmacist }\end{array}$ & $x$ & $P, V$ & $\begin{array}{l}\text { Self-Administration of } \\
\text { Medications } \\
\text { Programme (SAMP) } \\
\text { Medication list }\end{array}$ & $\begin{array}{l}3 \text { levels of SAMP } \\
\text { Level I: medication education by nurse and pharmacist, } \\
\text { provision of medication list with instructions and dose } \\
\text { times, nurse responsible for medication administration } \\
\text { Level 2: patient self-administrates medication under nurse } \\
\text { supervision } \\
\text { Level 3: independent patient self-administration }\end{array}$ & $x$ \\
\hline $\begin{array}{l}\text { Leguelinel- } \\
\text { Blache et al } \\
2015^{56}\end{array}$ & I Pharmacist & $x$ & $P, V$ & $\begin{array}{l}\text { Pharmacist service on } \\
\text { admission, during } \\
\text { admission, and at } \\
\text { discharge }\end{array}$ & $\begin{array}{l}\text { On admission: medication reconciliation to obtain best } \\
\text { possible medication history. Initial counselling occurs } \\
\text { during medication reconciliation process and determined } \\
\text { patient understanding and attitude towards medication use, } \\
\text { assessed barriers to adherence and social support, and } \\
\text { reviewed side effects, allergies, and intolerances. } \\
\text { During admission: routine prescription analysis to check } \\
\text { for dose, interactions, contraindications etc. } \\
\text { At discharge: reviewed discharge medication name, use, } \\
\text { dose, regimen, side effects etc. Sometimes supplemented } \\
\text { with illustrated medication schedule and drug containers. } \\
\text { Followed-up on barriers detected at admission. }\end{array}$ & $x$ \\
\hline Li et al $2016^{57}$ & I Pharmacist & $x$ & v & $\begin{array}{l}\text { Pharmacist service } \\
\text { during admission and } \\
\text { at discharge }\end{array}$ & $\begin{array}{l}\text { During admission: daily medication profile review and } \\
\text { creation of a best possible medication discharge list which } \\
\text { was later compared with the actual discharge list to identify } \\
\text { and resolve any discrepancies } \\
\text { At discharge: delivery of medications to the bedside. } \\
\text { Counseling on high-risk medications, select disease states, } \\
\text { and/or additional information given at the pharmacist's } \\
\text { discretion. Patient's discharge medication list is also sent to } \\
\text { follow-up providers. }\end{array}$ & $x$ \\
\hline $\begin{array}{l}\text { Louis- } \\
\text { Simonet et al } \\
2004^{58}\end{array}$ & I Physician & $x$ & $P, V$ & $\begin{array}{l}\text { Physician service at } \\
\text { discharge } \\
\text { Treatment card }\end{array}$ & $\begin{array}{l}\text { At discharge: patient-centered interview which employed } \\
\text { communication skills-based approach. Involved education } \\
\text { on medications, solicitation of questions and clarification of } \\
\text { treatment options. Standardized treatment card which } \\
\text { listed discharge medications was provided (see Table } 3 \text { for } \\
\text { details). }\end{array}$ & $\mathrm{x}$ \\
\hline
\end{tabular}


Table 2 (Continued).

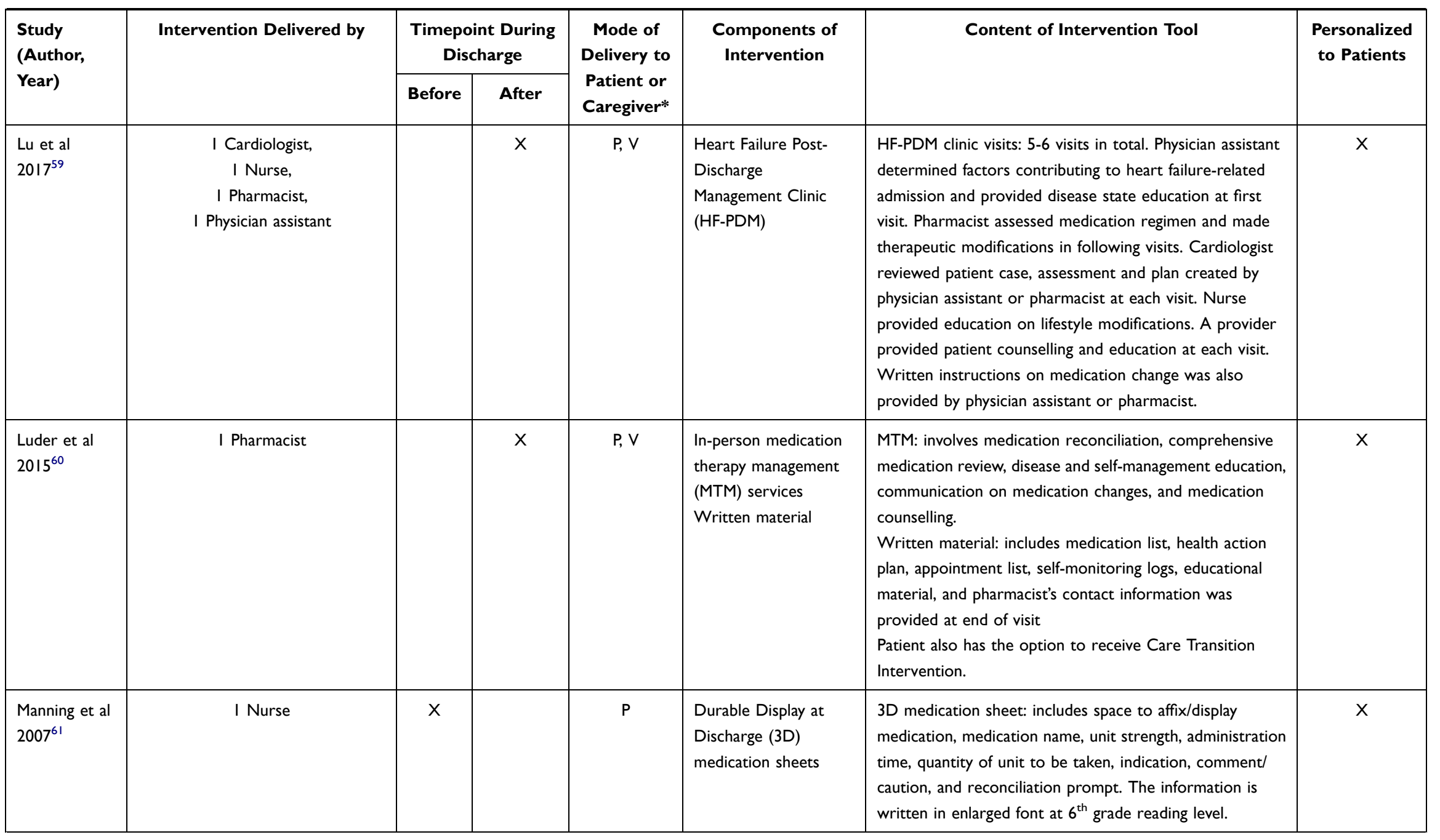




\begin{tabular}{|c|c|c|c|c|c|c|c|}
\hline $\begin{array}{l}\text { Marusic et al } \\
2018^{63}\end{array}$ & I Physician & $x$ & & $P, V$ & $\begin{array}{l}\text { Patient education } \\
\text { before discharge in } \\
\text { addition to standard } \\
\text { care } \\
\text { Information leaflet }\end{array}$ & $\begin{array}{l}\text { Patient education: provided for each discharge } \\
\text { prescription. Information include indication, dosage and } \\
\text { time of administration, importance of adherence, possible } \\
\text { consequences of non-adherence, possible side effects, and } \\
\text { measures to take if suspect adverse drug reaction. Leaflet } \\
\text { containing the same information in writing was also given. }\end{array}$ & $x$ \\
\hline $\begin{array}{l}\text { Marusic et al } \\
2013^{62}\end{array}$ & I Physician & $x$ & & $P, V$ & $\begin{array}{l}\text { Patient education } \\
\text { before discharge in } \\
\text { addition to standard } \\
\text { care } \\
\text { Information leaflet }\end{array}$ & $\begin{array}{l}\text { Patient education: provided for each discharge } \\
\text { prescription. Information include indication, dosage and } \\
\text { time of administration, importance of adherence, possible } \\
\text { consequences of non-adherence, and possible side effects. } \\
\text { Leaflet containing the same information in writing was also } \\
\text { given. }\end{array}$ & $x$ \\
\hline $\begin{array}{l}\text { McCarthy } \\
\text { et al } 2015^{64}\end{array}$ & $\begin{array}{l}\text { Physician, Research/Project team } \\
\text { (number unspecified) }\end{array}$ & $x$ & & $P, V$ & $\begin{array}{l}\text { MedSheets } \\
\text { Verbal information }\end{array}$ & $\begin{array}{l}\text { MedSheets: research assistant provided patient with one- } \\
\text { page medication information sheet on hydrocodone- } \\
\text { acetaminophen. Content include drug name, indication, } \\
\text { benefit, administration, discontinuation, etc. Information } \\
\text { was written in lower than } 8^{\text {th }} \text { grade reading level. } \\
\text { Verbal: The information on MedSheets was also read out } \\
\text { loud by a research assistant. Physicians addressed any } \\
\text { patient questions. }\end{array}$ & \\
\hline $\begin{array}{l}\text { Miller et al } \\
2016^{65}\end{array}$ & $\begin{array}{l}\text { Pharmacist, Pharmacy technician } \\
\text { (number unspecified) }\end{array}$ & & $x$ & $P, V$ & $\begin{array}{l}\text { Medication Therapy } \\
\text { Management (MTM) } \\
\text { service } \\
\text { Follow-up }\end{array}$ & $\begin{array}{l}\text { MTM: pharmacist provided comprehensive medication } \\
\text { review, personalized medication list, medication action } \\
\text { plan, and provider intervention (unspecified). Service } \\
\text { occurred over the phone. } \\
\text { Follow-up review call: pharmacist confirmed possession of } \\
\text { medication list and action plan (sent after initial call), } \\
\text { assessed for resolution of previously identified problems } \\
\text { and evaluated for any new issues. } \\
\text { Pharmacy technician provided administrative support and } \\
\text { gathered demographic and lifestyle information. }\end{array}$ & $x$ \\
\hline
\end{tabular}


Table 2 (Continued).

\begin{tabular}{|c|c|c|c|c|c|c|c|}
\hline \multirow{2}{*}{$\begin{array}{l}\text { Study } \\
\text { (Author, } \\
\text { Year) }\end{array}$} & \multirow[t]{2}{*}{ Intervention Delivered by } & \multicolumn{2}{|c|}{$\begin{array}{l}\text { Timepoint During } \\
\text { Discharge }\end{array}$} & \multirow{2}{*}{$\begin{array}{l}\text { Mode of } \\
\text { Delivery to } \\
\text { Patient or } \\
\text { Caregiver* }\end{array}$} & \multirow[t]{2}{*}{$\begin{array}{l}\text { Components of } \\
\text { Intervention }\end{array}$} & \multirow[t]{2}{*}{ Content of Intervention Tool } & \multirow[t]{2}{*}{$\begin{array}{c}\text { Personalized } \\
\text { to Patients }\end{array}$} \\
\hline & & Before & After & & & & \\
\hline $\begin{array}{l}\text { Moye et al } \\
2018^{66}\end{array}$ & $\begin{array}{l}\text { Pharmacist, Pharmacy residents, } \\
\text { Pharmacy students, Physician, } \\
\text { Nurse, Case manager } \\
\text { (number unspecified) }\end{array}$ & $x$ & $x$ & $\mathrm{P}, \mathrm{V}$ & $\begin{array}{l}\text { Pharmacy team service } \\
\text { upon patient in } \\
\text { addition to standard } \\
\text { care } \\
\text { Personalized binder } \\
\text { Follow-up }\end{array}$ & $\begin{array}{l}\text { Pharmacy team service: Medication reconciliation and } \\
\text { assessment of discrepancies with inpatient medication } \\
\text { orders. Discharge counselling on medications as well as the } \\
\text { importance of adherence (information was provided in } \\
\text { both verbal and written forms). } \\
\text { Personalized binder: provided to each patient. Contained } \\
\text { personalized medication record, weight and symptom log, } \\
\text { fluid intake log, salt intake log. } \\
\text { Follow-up: phone calls to ensure adherence and address } \\
\text { any issues that arose. }\end{array}$ & $x$ \\
\hline $\begin{array}{l}\text { Murphy et al } \\
2019^{67}\end{array}$ & $\begin{array}{l}\text { Cardiologist, Nurse, Pharmacists, } \\
\text { Pharmacy residents, Dietitians } \\
\text { (number unspecified) }\end{array}$ & $x$ & $x$ & $\mathrm{P}, \mathrm{V}$ & $\begin{array}{l}\text { Healthcare team } \\
\text { service during } \\
\text { admission and at } \\
\text { discharge } \\
\text { Follow-up }\end{array}$ & $\begin{array}{l}\text { Day 2-3 of admission: pharmacy personnel provided } \\
\text { education (signs and symptoms, healthy diet, post- } \\
\text { discharge medications) } \\
\text { Day 4-5: dietician counselled patient on heart healthy diet } \\
\text { Day of discharge: pharmacist assisted cardiologist and/ or } \\
\text { nurse practitioner with discharge medication reconciliation } \\
\text { and helped ensure the patient had a follow-up appointment } \\
\text { with their cardiologist within I week. } \\
\text { Throughout hospitalization: cardiologist and nurse } \\
\text { provided general disease state education } \\
\text { Follow up: } \\
\text { Week I: cardiologist appointment, nurse phone call and } \\
\text { then pharmacy resident phone call to ensure understanding } \\
\text { of and adherence to medications regimens } \\
\text { Week 2: Pharmacist medication therapy management } \\
\text { appointments. Patients provided with a list of current } \\
\text { medications and a medication action plan } \\
\text { Week 3: Dietician follow-up phone call }\end{array}$ & $x$ \\
\hline
\end{tabular}




\begin{tabular}{|c|c|c|c|c|c|c|c|}
\hline $\begin{array}{l}\text { Oliveira-Filho } \\
\text { et al } 2014^{68}\end{array}$ & I Research Pharmacist & $x$ & & $P, V$ & $\begin{array}{l}\text { Pharmacist service at } \\
\text { discharge } \\
\text { Drug treatment card }\end{array}$ & $\begin{array}{l}\text { At discharge: review of prescription to ensure } \\
\text { appropriateness and to minimize regimen complexity. } \\
\text { Counselling on disease and medications. Information on } \\
\text { therapeutic goals, self-monitoring of therapy, and adverse } \\
\text { reactions related to antihypertensive therapy were } \\
\text { provided. } \\
\text { Drug treatment card: Created by pharmacist after review } \\
\text { of patient data and prescription information. Adapted as } \\
\text { refrigerator magnet. Contains advice, dosage and } \\
\text { medication schedule. }\end{array}$ & $\mathrm{x}$ \\
\hline $\begin{array}{l}\text { Olives et al } \\
2016^{69}\end{array}$ & $\begin{array}{c}\text { Physician, } \\
\text { Text message alerts did not require } \\
\text { deliver by an individual }\end{array}$ & $x$ & $\begin{array}{l}\text { Not clear } \\
\text { when text } \\
\text { or voice } \\
\text { mail is } \\
\text { sent }\end{array}$ & $\mathrm{P}, \mathrm{V}, \mathrm{E}$ & $\begin{array}{l}2 \text { types: } \\
\text { l) standard of care in } \\
\text { addition to text } \\
\text { message } \\
\text { 2) standard of care in } \\
\text { addition to voice mail }\end{array}$ & $\begin{array}{l}\text { Text message: contained the treating physician's } \\
\text { instructions for self-administration of antibiotics. } \\
\text { Voice mail: contained spoken information from the treating } \\
\text { physician on self-administration of antibiotics. }\end{array}$ & $x$ \\
\hline $\begin{array}{l}\text { Phatak et al } \\
2016^{70}\end{array}$ & I Pharmacist & $x$ & $x$ & $\mathrm{v}$ & $\begin{array}{l}\text { Pharmacist service on } \\
\text { admission and at } \\
\text { discharge } \\
\text { Follow-up }\end{array}$ & $\begin{array}{l}\text { On admission: Face-to-face medication reconciliation } \\
\text { At discharge: created personalized medication plan and } \\
\text { addressed medication discrepancies before discharge. } \\
\text { Provided discharge instructions and medication counseling. } \\
\text { Follow-up: phone calls at } 3,14 \text {, and } 30 \text { days post-discharge } \\
\text { to assess for adverse drug events and medication errors. }\end{array}$ & $\mathrm{x}$ \\
\hline $\begin{array}{l}\text { Press et al } \\
2016^{71}\end{array}$ & $\begin{array}{l}\text { I Research educator, } \\
\text { I Research assistant }\end{array}$ & $x$ & & $P, V$ & $\begin{array}{l}\text { Teach-to-goal } \\
\text { intervention with } \\
\text { evaluation, } \\
\text { demonstration and } \\
\text { written information }\end{array}$ & $\begin{array}{l}\text { Evaluation and demonstration: I) Evaluation of participant } \\
\text { technique; 2) Demonstration of correct technique; 3) re- } \\
\text { assessment of participant technique using teach-back } \\
\text { method; 4) repeat of step 2-3 for up to two rounds; 5) final } \\
\text { evaluation of participant inhaler technique. } \\
\text { Written information: Provision of written instructions and } \\
\text { pamphlet describing basic information about asthma or } \\
\text { COPD (depending on participant condition). }\end{array}$ & \\
\hline
\end{tabular}


Table 2 (Continued).

\begin{tabular}{|c|c|c|c|c|c|c|c|}
\hline \multirow{2}{*}{$\begin{array}{l}\text { Study } \\
\text { (Author, } \\
\text { Year) }\end{array}$} & \multirow[t]{2}{*}{ Intervention Delivered by } & \multicolumn{2}{|c|}{$\begin{array}{l}\text { Timepoint During } \\
\text { Discharge }\end{array}$} & \multirow{2}{*}{$\begin{array}{l}\text { Mode of } \\
\text { Delivery to } \\
\text { Patient or } \\
\text { Caregiver* }\end{array}$} & \multirow[t]{2}{*}{$\begin{array}{l}\text { Components of } \\
\text { Intervention }\end{array}$} & \multirow[t]{2}{*}{ Content of Intervention Tool } & \multirow[t]{2}{*}{$\begin{array}{c}\text { Personalized } \\
\text { to Patients }\end{array}$} \\
\hline & & Before & After & & & & \\
\hline $\begin{array}{l}\text { Renaudin et al } \\
2017^{72}\end{array}$ & I Pharmacist or Pharmacy resident & $x$ & & $\mathrm{v}$ & $\begin{array}{l}\text { Pharmacist service at } \\
\text { admission and at } \\
\text { discharge }\end{array}$ & $\begin{array}{l}\text { At admission: medication reconciliation (comparison of } \\
\text { medication history with admission prescription to identify } \\
\text { discrepancies) and treatment review. } \\
\text { At discharge: medication reconciliation (comparison of } \\
\text { medication history with discharge prescription to identify } \\
\text { discrepancies), treatment review, and medication liaison } \\
\text { service (including comprehensive medication history, } \\
\text { counselling on medications, and discharge letter faxed to } \\
\text { the community pharmacist and general practitioner). }\end{array}$ & $x$ \\
\hline $\begin{array}{l}\text { Salmany et al } \\
2018^{73}\end{array}$ & I Research pharmacist & & $x$ & $\mathrm{v}$ & Follow-up & $\begin{array}{l}\text { Follow-up: phone call within } 72 \text { hours of discharge. } \\
\text { Whether patients were able to obtain all their medications } \\
\text { was assessed. Patients were inquired on medication } \\
\text { understanding and adverse reactions. Physician was notified } \\
\text { if patient reports medication-related adverse reactions. }\end{array}$ & $x$ \\
\hline $\begin{array}{l}\text { Sanii et al } \\
2016^{74}\end{array}$ & I Pharmacist & $x$ & $x$ & $\mathrm{P}, \mathrm{V}$ & $\begin{array}{l}\text { Pharmacist service at } \\
\text { discharge } \\
\text { Written education } \\
\text { material } \\
2 \text { Follow-ups }\end{array}$ & $\begin{array}{l}\text { At discharge: counselling on medications (indication, } \\
\text { interaction, administration, and side effect). Medication } \\
\text { reconciliation. Education on correct inhaler technique. } \\
\text { Provision of written asthma education materials. } \\
\text { Follow-up I: telephone call at } 2 \text { weeks after discharge to } \\
\text { determine medication discrepancies, medication } \\
\text { adherence, and possible adverse drug events. } \\
\text { Follow-up 2: meeting in clinic at I month after discharge to } \\
\text { assess discharge medications, inhaler technique, and } \\
\text { medication adherence. }\end{array}$ & $\mathrm{x}$ \\
\hline $\begin{array}{l}\text { Sarangarm } \\
\text { et al } 2012^{75}\end{array}$ & I Pharmacist & $x$ & & $\mathrm{v}$ & $\begin{array}{l}\text { Pharmacist service at } \\
\text { discharge } \\
\text { Follow-up }\end{array}$ & $\begin{array}{l}\text { At discharge: Medication reconciliation and identification of } \\
\text { potential drug therapy problems. Discharge counselling } \\
\text { with education on medication administration, adverse } \\
\text { reaction, and disease state. } \\
\text { Follow-up: phone call at } 36-72 \text { hours after discharge to } \\
\text { assess patient health and to identify and address drug- } \\
\text { related problems. }\end{array}$ & $x$ \\
\hline
\end{tabular}




\begin{tabular}{|c|c|c|c|c|c|c|c|}
\hline $\begin{array}{l}\text { Schwalm et al } \\
2015^{76}\end{array}$ & $\begin{array}{l}\text { Intervention did not require } \\
\text { delivery/administration by an } \\
\text { individual }\end{array}$ & & $x$ & $\mathrm{P}$ & Personalized letters & $\begin{array}{l}\text { Personalized letters: Sent to patient and family physician at } \\
\text { I, } 5,8 \text {, and II months after angiogram. Letter reviewed } \\
\text { role of each prescribed cardiac medication and encouraged } \\
\text { adherence. Letter encouraged family physician and } \\
\text { pharmacist participation in promoting adherence. } \\
\text { Information was written at } 6^{\text {th }} \text { grade reading level. Patient } \\
\text { received additional reminder postcard at } 2 \text { months. }\end{array}$ & $\mathrm{x}$ \\
\hline $\begin{array}{l}\text { Send et al } \\
2014^{77}\end{array}$ & I Physician & $x$ & & $\mathrm{P}$ & $\begin{array}{l}\text { Enhanced Medication } \\
\text { Plan (EMP) }\end{array}$ & $\begin{array}{l}\text { EMP: contained information on indications, step-by-step } \\
\text { administration instructions for complex processes such as } \\
\text { inhaler use, drug handling recommendations (e.g. storage, } \\
\text { drug-food staggering time). Generated using electronic } \\
\text { database with physician modification before printing. }\end{array}$ & $x$ \\
\hline $\begin{array}{l}\text { Shaver et al } \\
2019^{78}\end{array}$ & $\begin{array}{l}\text { I Advanced Pharmacy Practice } \\
\text { Experience (APPE) student, } \\
\text { I Pharmacist }\end{array}$ & $x$ & $x$ & $\mathrm{v}$ & $\begin{array}{l}\text { Transitions of Care } \\
\text { program in addition to } \\
\text { Prescriptions Plus }\end{array}$ & $\begin{array}{l}\text { Transitions of Care program: APPE student conducted } \\
\text { telephone call 2-7 days after discharge. Involved medication } \\
\text { reconciliation, assessment of adherence, and medication } \\
\text { counselling. Patients were encouraged to arrange and } \\
\text { attend follow-up appointments. Importance of adherence } \\
\text { and follow-up was emphasized. Pharmacist addressed } \\
\text { questions if needed. }\end{array}$ & $x$ \\
\hline $\begin{array}{l}\text { Shull et al } \\
2018^{79}\end{array}$ & $\begin{array}{l}\text { I Pharmacist, } \\
\text { I Bridge care coordinator }\end{array}$ & $x$ & $x$ & $\mathrm{v}$ & $\begin{array}{l}\text { Pharmacist service at } \\
\text { admission, during } \\
\text { admission, and at } \\
\text { discharge } \\
\text { Follow-up }\end{array}$ & $\begin{array}{l}\text { At admission, during admission, and at discharge: } \\
\text { pharmacist conducted I) medication reconciliation; 2) } \\
\text { medication therapy management and counselling; 3) } \\
\text { patient-centred education (involving teach-back); and 4) } \\
\text { assessed and addressed barriers to access to care. } \\
\text { During follow-up after discharge: bridge care coordinator } \\
\text { (and pharmacist if required) conducts follow-up phone call } \\
\text { weekly to encourage adherence, review discharge } \\
\text { instructions, address emerging issues. Potential home visit } \\
\text { offered to patient. }\end{array}$ & $x$ \\
\hline $\begin{array}{l}\text { Singh et al } \\
2018^{80}\end{array}$ & $\begin{array}{l}\text { Intervention did not require } \\
\text { delivery/ administration by an } \\
\text { individual }\end{array}$ & $x$ & & $\mathrm{P}$ & $\begin{array}{l}\text { Written discharge } \\
\text { instructions in addition } \\
\text { to usual care }\end{array}$ & $\begin{array}{l}\text { Written discharge instructions: information pamphlet with } \\
\text { instructions on ibuprofen, acetaminophen and opioid use } \\
\text { and management, postoperative pain expectations, and } \\
\text { recommendations for opioid medication indication, usage, } \\
\text { and disposal. }\end{array}$ & \\
\hline
\end{tabular}


Table 2 (Continued)

\begin{tabular}{|c|c|c|c|c|c|c|c|}
\hline \multirow{2}{*}{$\begin{array}{l}\text { Study } \\
\text { (Author, } \\
\text { Year) }\end{array}$} & \multirow[t]{2}{*}{ Intervention Delivered by } & \multicolumn{2}{|c|}{$\begin{array}{c}\text { Timepoint During } \\
\text { Discharge }\end{array}$} & \multirow{2}{*}{$\begin{array}{l}\text { Mode of } \\
\text { Delivery to } \\
\text { Patient or } \\
\text { Caregiver* }\end{array}$} & \multirow[t]{2}{*}{$\begin{array}{l}\text { Components of } \\
\text { Intervention }\end{array}$} & \multirow[t]{2}{*}{ Content of Intervention Tool } & \multirow[t]{2}{*}{$\begin{array}{c}\text { Personalized } \\
\text { to Patients }\end{array}$} \\
\hline & & Before & After & & & & \\
\hline $\begin{array}{l}\text { Sinha et al } \\
2019^{81}\end{array}$ & $\begin{array}{c}\text { Medical students } \\
\text { (number unspecified) }\end{array}$ & $x$ & & E & $\begin{array}{l}\text { Video discharge } \\
\text { education }\end{array}$ & $\begin{array}{l}\text { Video: addressed barriers to successful transition to home. } \\
\text { Topics were on medication reconciliation, medication } \\
\text { uncertainty, medication administration, medication } \\
\text { availability, and access to delivery of medication. Each } \\
\text { educational topic was followed by short multiple-choice } \\
\text { assessments. }\end{array}$ & \\
\hline $\begin{array}{l}\text { Smith et al } \\
2017^{82}\end{array}$ & $\begin{array}{l}\text { Pharmacist, Physician, Nutrition } \\
\text { course administration, GAP } \\
\text { transitional care services } \\
\text { (numbers unspecified) }\end{array}$ & $x$ & $x$ & $\mathrm{P}, \mathrm{V}$ & $\begin{array}{l}\text { Patient education } \\
\text { during hospitalization } \\
\text { Services after } \\
\text { discharge }\end{array}$ & $\begin{array}{l}\text { During hospitalization: patient education on admission } \\
\text { utilizing Heart Attack Program Guide and at discharge } \\
\text { utilizing teach-back strategies. Assessment of medications } \\
\text { prior to discharge. } \\
\text { After discharge: physician follow-up within one week. } \\
\text { Pharmacist follow-up on medications. Cardiac } \\
\text { rehabilitation. }\end{array}$ & $x$ \\
\hline $\begin{array}{l}\text { Tuttle et al } \\
2018^{83}\end{array}$ & I Nurse, I Pharmacist & $x$ & $x$ & $P, V$ & $\begin{array}{l}\text { Follow-up home visit } \\
\text { in addition to usual } \\
\text { care } \\
\text { Medication list }\end{array}$ & $\begin{array}{l}\text { Home visit: I-2 hour home within } 7 \text { days of discharge } \\
\text { provided by pharmacist. Involved I) comprehensive } \\
\text { medication review to identify and resolve medication- } \\
\text { related problems; } 2 \text { ) medication action plan in relation to } \\
\text { identified problems; } 3 \text { ) a personal medication list; } 4 \text { ) } \\
\text { counselling on proper medication use and avoidance of } \\
\text { contraindicated medication. }\end{array}$ & $x$ \\
\hline $\begin{array}{l}\text { Vuong et al } \\
2008^{84}\end{array}$ & I Community-liaison pharmacist & $x$ & $x$ & v & $\begin{array}{l}\text { Follow-up home visits } \\
\text { in addition to standard } \\
\text { care }\end{array}$ & $\begin{array}{l}\text { During home visits: assessment of patient medication } \\
\text { knowledge, administration techniques, medication supply } \\
\text { and storage, and compliance with medication regimen. } \\
\text { Additional education is provided when necessary. Expired } \\
\text { or no longer required medications were removed. }\end{array}$ & $x$ \\
\hline
\end{tabular}




\begin{tabular}{|l|l|l|l|l|l|l|}
\hline $\begin{array}{l}\text { Walker et al } \\
2009^{85}\end{array}$ & I Pharmacist & X & X & P, V & $\begin{array}{l}\text { Pharmacist service at } \\
\text { discharge } \\
\text { Follow-up }\end{array}$ & $\begin{array}{l}\text { At discharge: patient interviews, assessment of medication } \\
\text { therapy, medication reconciliation, communication of } \\
\text { medication monitoring follow-up plan, discharge } \\
\text { counselling on medications (e.g. medication instructions) } \\
\text { with written medication information, identification of } \\
\text { potential adherence concerns, communication with follow- } \\
\text { up provider to provide discharge medication list. } \\
\text { Follow-up: pharmacist telephone call at } 72 \text { hours and at } 30 \\
\text { days to non-Medicare Beneficiaries to address medication- } \\
\text { related problems and other patient concerns. }\end{array}$ \\
\hline $\begin{array}{l}\text { Zerafa et al } \\
2011^{86}\end{array}$ & I Pharmacist & $X$ & & $P, V$ & $\begin{array}{l}\text { Pharmacist service on } \\
\text { discharge } \\
\text { Discharge medication } \\
\text { chart }\end{array}$ & $\begin{array}{l}\text { On discharge: colored medication photographs and } \\
\text { discharge medication chart provided to educate patient on } \\
\text { identification of medication, medication doses, and } \\
\text { medication administration. Counselling on importance of } \\
\text { adherence with oral analgesia, exercise, and avoidance of } \\
\text { alcohol and smoking during recovery period. } \\
\text { Discharge medication chart: pictograms and information } \\
\text { on medications, regimens, administration instructions (see } \\
\text { Table 3 for details). }\end{array}$ \\
\hline
\end{tabular}

Notes: *P, print; $\mathrm{V}$, verbal; E, electronic. 
Table 3 UCD-I ${ }^{26}$ Criteria of Included Studies

\begin{tabular}{|c|c|c|c|c|c|c|c|c|c|c|c|c|c|c|c|}
\hline \multirow[t]{2}{*}{ UCD-II Criterion } & \multicolumn{15}{|c|}{ Study (Author, Year) } \\
\hline & $\begin{array}{l}\text { Anderegg } \\
\text { et al, } \\
2013^{29}\end{array}$ & $\begin{array}{l}\text { Baky } \\
\text { et al, } \\
2018^{31}\end{array}$ & $\begin{array}{l}\text { Barnason } \\
\text { et al, } \\
2010^{33}\end{array}$ & $\begin{array}{l}\text { Bell } \\
\text { et al, } \\
2016^{34}\end{array}$ & $\begin{array}{l}\text { Bolas } \\
\text { et al, } \\
2004^{35}\end{array}$ & $\begin{array}{l}\text { Bonetti } \\
\text { et al, } \\
2018^{36}\end{array}$ & $\begin{array}{l}\text { Cordasco } \\
\text { et al, } \\
2009^{42}\end{array}$ & $\begin{array}{l}\text { Ducharme } \\
\text { et al, } \\
2011^{47}\end{array}$ & $\begin{array}{l}\text { Jones } \\
\text { et al, } \\
2018^{51}\end{array}$ & $\begin{array}{l}\text { Kaestli } \\
\text { et al, } \\
2016^{52}\end{array}$ & $\begin{array}{l}\text { McCarthy } \\
\text { et al, } \\
2015^{64}\end{array}$ & $\begin{array}{l}\text { Schwalm } \\
\text { et al, } \\
2015^{76}\end{array}$ & $\begin{array}{l}\text { Sinha } \\
\text { et al, } \\
2019^{81}\end{array}$ & $\begin{array}{l}\text { Smith } \\
\text { et al, } \\
2017^{82}\end{array}$ & $\begin{array}{l}\text { Zerafa } \\
\text { et al, } \\
2011^{86}\end{array}$ \\
\hline $\begin{array}{l}\text { Were potential end users (eg, } \\
\text { patients, caregivers, family and } \\
\text { friends, surrogates) involved in any } \\
\text { steps to help understand users and } \\
\text { their needs? }\end{array}$ & $x$ & & $x$ & $x$ & & & $x$ & $x$ & & $x$ & & & $x$ & & \\
\hline $\begin{array}{l}\text { Were potential end users (eg, } \\
\text { patients, caregivers, family and } \\
\text { friends, surrogates) involved in any } \\
\text { steps of designing, developing, and/or } \\
\text { refining a prototype? }\end{array}$ & & $x$ & & & & & $x$ & $x$ & & & & $x$ & $\mathrm{x}$ & & $x$ \\
\hline $\begin{array}{l}\text { Did the development process have } 3 \\
\text { or more iterative cycles? }\end{array}$ & & & & & & $x$ & & $x$ & & & & & $x$ & & \\
\hline $\begin{array}{l}\text { Were changes between iterative } \\
\text { cycles explicitly reported in any way? }\end{array}$ & $x$ & & & & $x$ & $x$ & & $x$ & & & & & & & \\
\hline $\begin{array}{l}\text { Were health professionals consulted } \\
\text { at any point before a first prototype } \\
\text { was developed? }\end{array}$ & $x$ & & & & & & & $x$ & $x$ & $x$ & $x$ & $x$ & $x$ & & \\
\hline $\begin{array}{l}\text { Were health professionals consulted } \\
\text { between initial and final prototypes? }\end{array}$ & $x$ & & & & $x$ & & $x$ & $x$ & & & $x$ & $x$ & $x$ & $x$ & \\
\hline Was an expert panel involved? & & & & $x$ & & & & & & & & & & & \\
\hline $\begin{array}{l}\text { Were potential end users (eg, } \\
\text { patients, caregivers, family and } \\
\text { friends, surrogates) observed using } \\
\text { the tool in any way? }\end{array}$ & & & $\mathrm{x}$ & $x$ & $x$ & $x$ & $x$ & $x$ & & & & $x$ & $x$ & & $x$ \\
\hline $\begin{array}{l}\text { Were potential end users (eg, } \\
\text { patients, caregivers, family and } \\
\text { friends, surrogates) involved in any } \\
\text { steps intended to evaluate } \\
\text { prototypes or a final version of the } \\
\text { tool? }\end{array}$ & $x$ & $x$ & $x$ & $x$ & $x$ & $x$ & $x$ & $x$ & $x$ & $x$ & $x$ & $x$ & $x$ & $x$ & $x$ \\
\hline $\begin{array}{l}\text { Were potential end users (eg, } \\
\text { patients, caregivers, family and } \\
\text { friends, surrogates) asked their } \\
\text { opinions of the tool in any way? }\end{array}$ & & & & & $x$ & & $x$ & $x$ & & $x$ & & & $x$ & & \\
\hline $\begin{array}{l}\text { Were health professionals asked } \\
\text { their opinion of the tool at any } \\
\text { point? }\end{array}$ & $x$ & & & & $x$ & & $x$ & $x$ & & $x$ & & $x$ & $x$ & & \\
\hline
\end{tabular}


Table 4 Characteristics of Written Information in Physical/Electronic Interventions*

\begin{tabular}{|c|c|c|c|c|}
\hline $\begin{array}{l}\text { Study (Author, } \\
\text { Year) }\end{array}$ & Pictograms & $\begin{array}{l}\text { Medication } \\
\text { Schedule }\end{array}$ & $\begin{array}{l}\text { Colored } \\
\text { Coded }\end{array}$ & Content \\
\hline $\begin{array}{l}\text { Al-Hashar et al, } \\
2018^{27}\end{array}$ & No & No & No & -Generic name of medications \\
\hline $\begin{array}{l}\text { Anderegg et al, } \\
2013^{29}\end{array}$ & No & No & No & $\begin{array}{l}\text {-Medication format (eg, brand name, generic) unspecified } \\
\text {-Reasons for use }\end{array}$ \\
\hline $\begin{array}{l}\text { Barnason et al, } \\
2010^{33}\end{array}$ & No & Yes & No & $\begin{array}{l}\text {-Medication format (eg, brand name, generic) unspecified } \\
\text {-Reasons for use }\end{array}$ \\
\hline Bell et al, $2016^{34}$ & $\begin{array}{l}\text { Yes - Used to indicate time of dosing (eg, morning, } \\
\text { afternoon, evening, bedtime icons) and indication }\end{array}$ & Yes & No & $\begin{array}{l}\text {-Brand name, generic name } \\
\text {-Charts for medications to start, stop or continue } \\
\text {-Reasons for use } \\
\text {-Timing and frequency of administration } \\
\text {-Side effects }\end{array}$ \\
\hline Bolas et al, $2004^{35}$ & No & Yes & No & $\begin{array}{l}\text {-Brand name, generic name } \\
\text {-Medications to take home } \\
\text {-Reasons for use } \\
\text {-Timing of administration }\end{array}$ \\
\hline $\begin{array}{l}\text { Bonetti et al, } \\
2018^{36}\end{array}$ & No & Yes & No & $\begin{array}{l}\text {-Medication format (eg, brand name, generic) unspecified } \\
\text {-Reasons for use } \\
\text {-Route, timing, frequency of administration } \\
\text {-Side effects }\end{array}$ \\
\hline $\begin{array}{l}\text { Budiman et al, } \\
2016^{37}\end{array}$ & No & No & No & $\begin{array}{l}\text {-Brand name, generic name } \\
\text {-Medications to start and continue } \\
\text {-Reasons for use } \\
\text {-Timing and frequency of administration }\end{array}$ \\
\hline Chan et al, $2018^{40}$ & No & Yes & Yes & $\begin{array}{l}\text {-Brand name, generic name } \\
\text {-Listed paracetamol, ibuprofen, oxycodone } \\
\text {-Reasons for use } \\
\text {-Dosage form } \\
\text {-Timing and frequency of administration } \\
\text {-Side effects } \\
\text {-Drug/food interactions }\end{array}$ \\
\hline $\begin{array}{l}\text { Cordasco et al, } \\
2009^{43}\end{array}$ & $\begin{array}{l}\text { Yes - used to indicate time of dosing in the day (eg, } \\
\text { morning, afternoon, bedtime), Medication (eg, picture of } \\
\text { the tablet/capsule), drug administration (eg, with/ } \\
\text { without food, indication (eg, image of heart) }\end{array}$ & Yes & Yes & $\begin{array}{l}\text {-Brand name, generic name } \\
\text {-Listed prescribed medications } \\
\text {-Reasons for use } \\
\text {-Dosage form } \\
\text {-Timing and frequency of administration }\end{array}$ \\
\hline Davis et al, $2012^{45}$ & Yes & Yes & No & -Details unspecified \\
\hline $\begin{array}{l}\text { Ducharme et al, } \\
2011^{47}\end{array}$ & $\begin{array}{l}\text { Yes - Included icons of individuals who are happy, okay, } \\
\text { sad to indicate what one should do based on how well } \\
\text { one's asthma control is }\end{array}$ & No & Yes & $\begin{array}{l}\text {-Medication format (eg, brand name, generic) unspecified } \\
\text {-Reasons for use } \\
\text {-Dosage form } \\
\text {-Frequency of administration }\end{array}$ \\
\hline Jiang et al, $2016^{50}$ & Yes & No & No & -Side effects \\
\hline $\begin{array}{l}\text { Kaestli et al, } \\
2016^{52}\end{array}$ & No & No & No & $\begin{array}{l}\text {-Medication format (eg, brand name, generic) unspecified } \\
\text {-Reasons for use } \\
\text {-Dosage form } \\
\text {-Timing and frequency of administration } \\
\text {-Side effects } \\
\text {-Drug/food interactions }\end{array}$ \\
\hline
\end{tabular}

(Continued) 
Table 4 (Continued).

\begin{tabular}{|c|c|c|c|c|}
\hline $\begin{array}{l}\text { Study (Author, } \\
\text { Year) }\end{array}$ & Pictograms & $\begin{array}{l}\text { Medication } \\
\text { Schedule }\end{array}$ & $\begin{array}{l}\text { Colored } \\
\text { Coded }\end{array}$ & Content \\
\hline $\begin{array}{l}\text { Kapoor et al, } \\
2019^{53}\end{array}$ & $\begin{array}{l}\text { Yes - Used to indicate time of dosing during the day (eg } \\
\text { morning, afternoon, evening, bedtime icons). Number of } \\
\text { pills taken per dose. Medication (eg picture of the tablet/ } \\
\text { capsule). Indication }\end{array}$ & Yes & Yes & $\begin{array}{l}\text {-Common names of medications } \\
\text {-Medications to start and continue } \\
\text {-Reasons for use } \\
\text {-Dosage form } \\
\text {-Timing and frequency of administration }\end{array}$ \\
\hline Lam et al, $2011^{55}$ & No & No & No & $\begin{array}{l}\text {-Medication format (eg, brand name, generic) unspecified } \\
\text {-Timing of administration }\end{array}$ \\
\hline $\begin{array}{l}\text { Leguelinel-Blache } \\
\text { et al, } 2015^{56}\end{array}$ & No & Yes & No & $\begin{array}{l}\text {-Medication format (eg, brand name, generic) unspecified } \\
\text {-Medications to start and continue } \\
\text {-Reasons for use } \\
\text {-Frequency of administration } \\
\text {-Side effects }\end{array}$ \\
\hline $\begin{array}{l}\text { Louis-Simonet } \\
\text { et al, } 2004^{58}\end{array}$ & No & No & No & $\begin{array}{l}\text {-Medication format (eg, brand name, generic) unspecified } \\
\text {-Listed all discharge medications } \\
\text {-Reasons for use } \\
\text {-Frequency of administration } \\
\text {-Side effects }\end{array}$ \\
\hline Luder et al, $2015^{60}$ & No & No & No & $\begin{array}{l}\text {-Medication format (eg, brand name, generic) unspecified } \\
\text {-Medications to start and continue } \\
\text {-Timing and frequency of administration }\end{array}$ \\
\hline $\begin{array}{l}\text { Manning et al, } \\
2007^{61}\end{array}$ & No & Yes & Yes & $\begin{array}{l}\text {-Brand name, generic name } \\
\text {-Medications to start, stop, and continue } \\
\text {-Reasons for use } \\
\text {-Dosage forms } \\
\text {-Timing and frequency of administration } \\
\text {-Side effects } \\
\text {-Drug/food interactions }\end{array}$ \\
\hline $\begin{array}{l}\text { Marusic et al, } \\
2018^{63}\end{array}$ & No & No & No & $\begin{array}{l}\text {-Medication format (eg, brand name, generic) unspecified } \\
\text {-Reasons for use } \\
\text {-Timing of administration } \\
\text {-Side effects }\end{array}$ \\
\hline $\begin{array}{l}\text { Marusic et al, } \\
2013^{62}\end{array}$ & No & No & No & $\begin{array}{l}\text {-Medication format (eg, brand name, generic) unspecified } \\
\text {-Reasons for use } \\
\text {-Timing of administration } \\
\text {-Side effects }\end{array}$ \\
\hline $\begin{array}{l}\text { McCarthy et al, } \\
2015^{64}\end{array}$ & Unspecified & Unspecified & Unspecified & $\begin{array}{l}\text {-Medication format (eg, brand name, generic) unspecified } \\
\text {-Reasons for use } \\
\text {-Timing and frequency of administration } \\
\text {-Side effects }\end{array}$ \\
\hline Moye et al, $2018^{66}$ & No & Yes & No & $\begin{array}{l}\text {-Medication format (eg, brand name, generic) unspecified } \\
\text {-Listed prescribed medications } \\
\text {-Reasons for use } \\
\text {-Frequency of administration } \\
\text {-Side effects }\end{array}$ \\
\hline Send et al, $2014^{77}$ & $\begin{array}{l}\text { Yes - used to provide information on dosage form and } \\
\text { drug administration (eg, with/without food, shake well } \\
\text { before use, do not split tablet, protect from heat/light } \\
\text { etc.) }\end{array}$ & Yes & Yes & $\begin{array}{l}\text {-Brand name, generic name, } \\
\text {-Medications to start and continue } \\
\text {-Reasons for use } \\
\text {-Dosage form } \\
\text {-Timing and frequency of administration }\end{array}$ \\
\hline
\end{tabular}

(Continued) 
Table 4 (Continued).

\begin{tabular}{|c|c|c|c|c|}
\hline $\begin{array}{l}\text { Study (Author, } \\
\text { Year) }\end{array}$ & Pictograms & $\begin{array}{l}\text { Medication } \\
\text { Schedule }\end{array}$ & $\begin{array}{l}\text { Colored } \\
\text { Coded }\end{array}$ & Content \\
\hline $\begin{array}{l}\text { Schwalm et al, } \\
2015^{76}\end{array}$ & No & No & No & $\begin{array}{l}\text {-Brand name, generic name } \\
\text {-Listed cardiac medications } \\
\text {-Reasons for use }\end{array}$ \\
\hline Singh et al, $2018^{80}$ & Yes & Yes & Yes & $\begin{array}{l}\text {-Brand name, generic name of acetaminophen and ibuprofen } \\
\text {-Reasons for use } \\
\text {-Medications to start } \\
\text {-Frequency of administration }\end{array}$ \\
\hline $\begin{array}{l}\text { Zerafa et al, } \\
2011^{86}\end{array}$ & $\begin{array}{l}\text { Yes - Used to indicate time of dosing (eg, morning, } \\
\text { afternoon, evening, bedtime icons). Pictures of } \\
\text { prescribed medication }\end{array}$ & Yes & Yes & $\begin{array}{l}\text {-Brand name, generic name } \\
\text {-Medications to start and continue } \\
\text {-Dosage form } \\
\text {-Timing and frequency of administration }\end{array}$ \\
\hline
\end{tabular}

Notes: * Studies with interventions that included physical/electronic components but did not specify the characteristics listed herein are not included in this table. These studies include: Cabilan et al, 2019, Chedepudi et al, 2017, Christy et al, 2016, De La Fuente et al, 20II, Lu et al, 2017, Miller et al, 20I6, Murphy et al, 20I9, Oliveira-Filho et al, 2014, Press et al, 2016, Sanii et al, 2016, Smith et al, 2017, Tuttle et al, 2018, Walker et al, 2009.

\section{Personalization}

Interventions were considered "personalized" if they contained any information specific to the patient, rather than a particular medication or medical condition. Most of the interventions $(83.3 \%, \mathrm{n}=50 / 60)$ were personalized to the patient. ${ }^{27-30,32-38,42-49,51,53-63,65-70,72-79,82-86}$ The most common methods of personalization were including the patient's specific medications list. However, only $13.3 \%(\mathrm{n}=8 / 60)$ involved assessment of the patient's health priorities (eg if they wanted the medication list in a particular language). ${ }^{33,34,36,43,45,47,49,79}$ Even fewer $(11.7 \%, \mathrm{n}=7 / 60)$ reported integrating the patient's values and preferences into the care plan. ${ }^{33,34,36,43,45,49,79}$ Few interventions $(20.0 \%, n=12 / 60)$ addressed the patient's ability to access medications financially in the community. ${ }^{32-34,36,37,48,51,56,70,73,75,79}$

\section{Process}

\section{Patient Involvement}

A large portion $(81.7 \%, \mathrm{n}=49)$ of interventions included patient counselling. ${ }^{28-46,48-51,53,55-60,62,63,66-75,78,79,82-86}$ Of these interventions, $53.1 \%(n=26 / 49)$ directly involved the patient during intervention delivery through strategies such as assessing patient understanding during the delivery or engaging patients in creation of a management plan. ${ }^{33,34,36-}$ $38,42,44,45,49,53,55,56,58,60,66,68,70,71,73,74,79,82-86$ Twenty-two (44.9\%) interventions assessed the patient for understanding,$33,34,37,38,42,45,49,56,60,66,68,70,71,73,74,79,82-86$ while $22.4 \%$ ( $\mathrm{n}=11 / 49)$ of these interventions specifically used teach-back (eg confirm patients' understanding by asking them to "teach it back" to the health-care provider), ${ }^{34,38,42,45,49,53,71,74,79,82,83}$ and $12.2 \%(n=6 / 49)$ assessed the patient's health priorities (eg values, preferences). ${ }^{33,34,36,45,49,79}$ Other strategies used included increasing responsibility based on patient's successful compliance, patient's own management plan using a template, and positive reinforcement.

\section{Timing}

The majority of interventions were delivered to the patient before leaving the hospital $(51.7 \%, \mathrm{n}=31)$. $^{28,29,31,32,35,38-}$ $41,43,46,47,49,50,52,55-58,61-64,68,71,72,75,77,80,81,86$ Thirty percent $(\mathrm{n}=18)$ of interventions had elements delivered before and after the patients left the hospital,,$^{30,34,36,37,42,45,48,51,66,67,70,74,78,79,82-85} 16.7 \%(\mathrm{n}=10)$ of interventions were delivered exclusively after the patients left the hospital, ${ }^{27,33,44,53,54,59,60,65,73,76}$ and for one of the studies, it was not clear when the intervention was delivered to the patient based on the manuscript. ${ }^{69}$

\section{Healthcare Providers Involved and Number of Providers}

Many interventions involved delivery from pharmacists $(61.7 \%, \mathrm{n}=37),{ }^{27-32,34-38,41,42,46,48,51-53,55-57,59,65-68,70,72-75,78,79,82,84-86}$ physicians $(25.0 \%, \mathrm{n}=15){ }^{27,31,40,47,58-60,62-64,66,67,69,77,82}$ and nurses $(25.0 \%, \mathrm{n}=15) .{ }^{30,32,33,39,43,44,49,50,53,55,59,61,66,67,83}$ 
Table 5 Outcome Measures of Studies*

\begin{tabular}{|c|c|c|c|}
\hline Outcome & $\begin{array}{l}\text { Number of Studies with } \\
\text { Outcome (\% of All Studies, n / } \\
60 \text { Studies) }\end{array}$ & $\begin{array}{c}\text { Number of Studies With Impact } \\
\text { Demonstrated (\% Studies Within } \\
\text { Category) }\end{array}$ & Study \\
\hline Patient and caregiver knowledge/ comprehension & $18(30)$ & $12(67)$ & 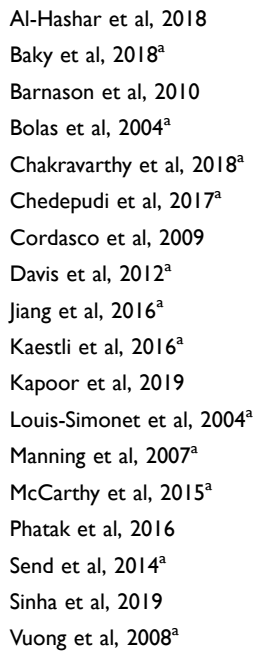 \\
\hline $\begin{array}{l}\text { Patient and caregiver attitudes (e.g., satisfaction, } \\
\text { preferences, values) and confidence (e.g., self- } \\
\text { efficacy) }\end{array}$ & $17(28)$ & $7(4 \mid)$ & 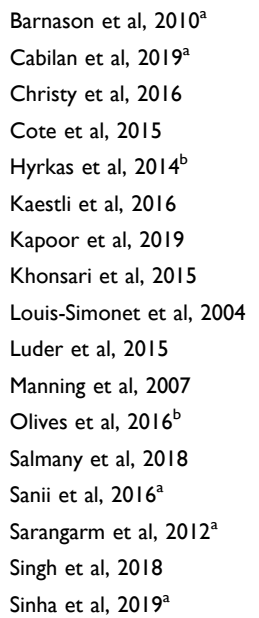 \\
\hline
\end{tabular}

(Continued)

Thirteen interventions (21.7\%) involved delivery from multiple health-care providers. ${ }^{27,30-32,53,55,59,65-67,79,82,83}$ Only $26.7 \%(\mathrm{n}=$ $16 / 60)$ studies reported training the staff administering the intervention. $33,34,36,38,40,43,45,48,49,53,60,64,67,68,71,83$ Twenty percent $(n=$ 12) of interventions were shared with other health-care practitioners outside the intervention setting. ${ }^{35,47,48,53,57,60,72,73,76,83-85}$

\section{Reported Outcomes}

"Table 5: Outcome Measure of Studies" presents details regarding outcome measures ie, which of the included studies assessed a particular outcome category, and those that demonstrated a clinically significant impact in the intervention group. Overall, 63\% (38/60) 27-38,42,45-49,51,54,56,57,60,62,65-68,70,71,73-75,78,79,81,83,85 of included studies reported health system outcomes (eg readmission rate, mortality rate) and 52\% $(31 / 60)^{28,30,33,36,37,40,43-47,49,50,53-56,58,59,62-}$ $64,68,69,71,74,75,80,84,86$ explored patient and caregiver behavior and experience (eg behaviours related to adherence, lifestyle management). Thirty-percent $(18 / 60)^{28,31,33,35,39,41,43,45,50,52,53,58,61,64,70,77,81,84}$ examined patient and caregiver knowledge/comprehension (eg, understanding of condition and medication), 28\% (17/60) $33,38,42,46,49,52-54,58,60,61,69,73-$ 75,80,81, reported patient and caregiver attitudes and confidence (eg, satisfaction, preferences, values and self-efficacy; and 
Table 5 (Continued).

\begin{tabular}{|c|c|c|c|}
\hline Outcome & $\begin{array}{c}\text { Number of Studies with } \\
\text { Outcome (\% of All Studies, } n \text { / } \\
60 \text { Studies) }\end{array}$ & $\begin{array}{c}\text { Number of Studies With Impact } \\
\text { Demonstrated (\% Studies Within } \\
\text { Category) }\end{array}$ & Study \\
\hline $\begin{array}{l}\text { Patient and caregiver behavior and experience } \\
\text { (e.g., adherence, self-management) }\end{array}$ & $31(52)$ & $21(68)$ & 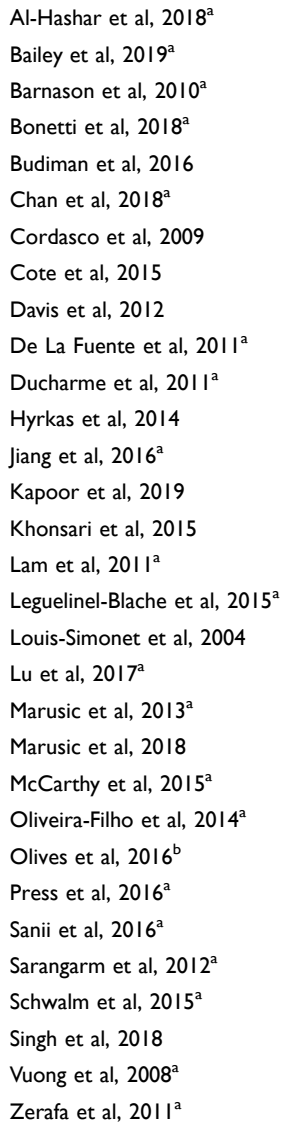 \\
\hline
\end{tabular}

(Continued)

$27 \%(16 / 60)^{30,31,35,40,43,50,51,54,63,65,70,75,77,79,82,85}$ explored other outcome measures (eg, health-care provider insights, economic impact).

With respect to included studies reporting significant findings, $68 \%$ (21 of 31 studies $)^{28,30,33,36,40,46,47,50,55,56,59,62,64,68,69,71,74-}$ $76,84,86$ of studies reported significant impact on patient and caregiver behavior and experience. Sixty-seven percent (12 of 18 studies) $)^{31,35,39,41,45,50,52,58,61,64,77,84}$ assessing patient and caregiver knowledge, 55\% (21 of 38 studies $)^{27-33,35,36,47-}$ $49,60,62,66,70,71,75,78,79,85$ assessing health system outcomes, 44\% (7 of 16 studies) ${ }^{30,31,50,51,54,77,85}$ assessing other outcome measures (eg health-care provider insights, economic impact) and 41\% (7 of 17 studies) $33,38,49,69,74,75,81$ assessing patient and caregiver attitudes and confidence demonstrated a clinically significant impact in the intervention group.

\section{Discussion}

Our rapid scoping review of medication support-related $\mathrm{TiC}$ interventions identified 60 relevant studies. The majority of studies were randomized controlled trials involving verbal counselling and/or physical document delivered to the patient before discharge. Most studies assessed health system outcomes and patient/caregiver behavior and experience, with the majority of studies showing a significant improvement in these outcomes. We identified four key findings, which differ from previous reviews on the topic of medication support and care transitions: ${ }^{87,88} 1$ ) the need for improved reporting of intervention development and characteristics; 2) the need to include high-risk populations when designing studies; 3) the 
Table 5 (Continued).

\begin{tabular}{|c|c|c|c|}
\hline Outcome & $\begin{array}{c}\text { Number of Studies with } \\
\text { Outcome (\% of All Studies, n / } \\
60 \text { Studies) }\end{array}$ & $\begin{array}{c}\text { Number of Studies With Impact } \\
\text { Demonstrated (\% Studies Within } \\
\text { Category) }\end{array}$ & Study \\
\hline $\begin{array}{l}\text { Health system outcomes (e.g., readmission, } \\
\text { mortality) }\end{array}$ & $38(63)$ & $21(55)$ & 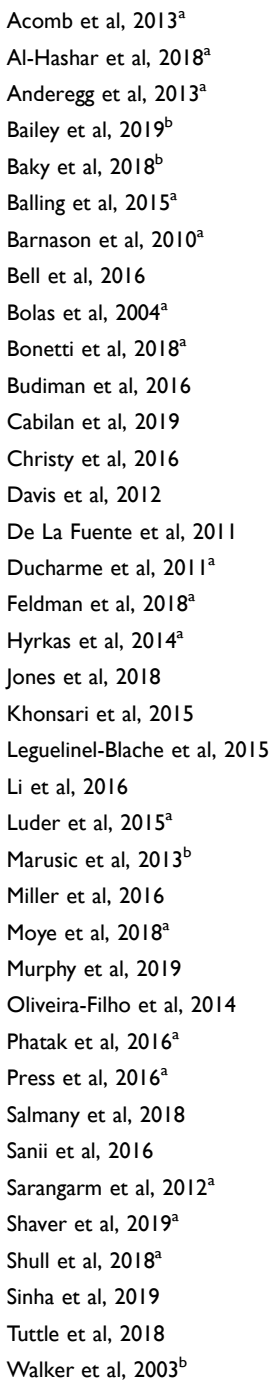 \\
\hline $\begin{array}{l}\text { Other (e.g., economic analysis, health care } \\
\text { provider insights/ interview/ survey) }\end{array}$ & $16(27)$ & 7 (44) & 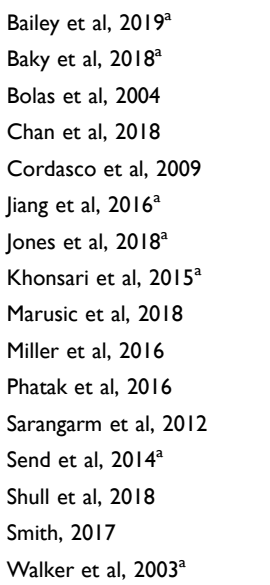 \\
\hline
\end{tabular}

Notes: *Renaudin et al, 2017 not included as results are not available (trial protocol). ${ }^{a}$ Significant finding in this study. ${ }^{b}$ Significant differences found among subgroups of population. 
potential for future research to apply user-centred design approaches to intervention development, 4) the opportunity for future studies to examine electronic interventions.

Our first key finding is that included studies lacked reporting on the development and characteristics of interventions. The majority of included studies failed to mention the development of their tool entirely, making it difficult to understand the rationale and elements of the interventions, and to assess whether the interventions addressed patient needs. Further, studies were lacking in their description of interventions for both physical and counselling interventions. For example, Lam 2011 mentioned a "medication list with detailed instructions" but did not specify these details. ${ }^{55}$ Also, Chakravarthy 2018 suggested that "discharge instructions" were provided by nursing staff but did not describe the content or components of the instructions. ${ }^{39}$

Importantly, these gaps in reporting make it difficult to discern what was tested, thereby diminishing the replicability of particular studies in situations where beneficial impact in the intervention group was demonstrated. We recommend increased use of the TIDieR reporting guideline to ensure that authors provide sufficient details when describing their intervention. ${ }^{89}$ Further, similarly detailed reporting of the elements of usual care comparators will help readers better understand the "baseline" and assess applicability to their own health systems. This review identified that few staff administering interventions were trained prior to the study. It was unclear whether training was not reported or if it was not provided. A possible explanation may be that interventions were delivered by health-care providers performing their regular scope of practice and did not require additional training. Regardless, additional reporting in publications regarding training, or the lack thereof, is encouraged.

Second, our scoping review identified an important gap with respect to patient populations that have been studied. Very few studies included or considered at-risk populations beyond older adults, such as patients with low literacy, language barriers, or disabilities, in their patient selection or sub-group analysis. These groups are at increased risk of adverse events and more likely to experience systemic barriers to care. ${ }^{8,10,90}$ Based on the theory of design by exception, considering these "extreme" users when designing an intervention, instead of designing for the average, will result in more equitable interventions that are compatible with the large majority of users and does so more efficiently than other mainstream design approaches. ${ }^{13,91}$

Third, we found that very few studies met the UCD-11 criteria, which is a way to describe an intervention's user centredness ie, explores end user involvement in design, development and refinement stages of an intervention. ${ }^{26}$ This may be partially but not fully explained by poor reporting of how interventions were developed. The strengths of participatory action and user-centered design approaches when developing complex interventions are increasingly recognized, as evidenced, for example, by their inclusion in recent UK Medical Research Council guidance. ${ }^{92}$ Members of our research team have successfully used these approaches to engage patients, caregivers, and health-care providers in co-developing patient-oriented discharge instructions, which has subsequently undergone wide-scale implementation and evaluation at the provincial level with positive results. ${ }^{90,93}$ The application of these approaches when developing medication-focused interventions at care transitions is an important opportunity for researchers to explore.

Finally, we note that most interventions included in this review involved providing written information and verbal instructions or education to patients and caregivers. Electronic interventions were the least frequently used. The paucity of electronic interventions was somewhat unexpected considering the shift toward technology in health care. Considering that the technology is available for various tools, such as medication time reminder, missed medication alerts, applications across multiple devices and much more, future assessment and use of these tools is another opportunity for future studies.

\section{Strengths and Limitations}

Limitations of this study are consistent with those known for rapid reviews. ${ }^{94}$ After piloting the process for title and abstract screening to attain agreement on $5 \%$ of the studies, subsequent studies were assessed for eligibility by one reviewer. Our review was limited to articles published between January 2004 and July 2019 in English, articles indexed in well-known databases, and those known to context experts. Grey literature sources were not searched and as an optional component of scoping reviews, risk of bias for included studies was not assessed. 
Despite these limitations, our study has several strengths building on previous research conducted examining medication interventions during care transitions. For example, Tomlinson et $\mathrm{al}^{87}$ conducted a systematic review and meta-analysis of randomized controlled trials evaluating interventions that support medication continuity in older adults at or started within one month of discharge from hospital. Consistent with scoping review methods, we used a broader search strategy (ie, included more constructs), and included study designs beyond randomized controlled trials, more eligible populations, and studies with a range of outcomes. Another systematic review and meta-analysis by Daliri et al ${ }^{88}$ examining the impact of prospective studies of medication-related interventions at care transitions on outcomes, is complementary to our review. They used meta-analyses to estimate the impact of interventions on outcomes while we focused on describing patient and caregiver-centered medication support interventions and how user-centered design elements were considered in the development of these interventions. Our study builds on their review by expanding the breadth of study designs and outcomes eligible for inclusion.

\section{Conclusion}

There is global interest in understanding the components and impact of medication-related interventions on patient outcomes during care transitions, as evidenced by recent systematic reviews exploring this topic. In this rapid scoping review, focused on describing patient and caregiver-centered medication support interventions and the role of usercentred design, the majority of studies included were randomized controlled trials involving verbal counselling and/or physical document delivered to the patient before discharge. Most studies selected patients based on age or medical condition, and few studies considered patients at high-risk of adverse events. Few studies reported on the development of the intervention. The majority of studies included multiple components, most commonly involving verbal or physical modalities, rather than electronic interventions. With respect to outcomes, most studies assessed health system outcomes and patient and caregiver behavior and experiences. Less commonly explored were patient and caregiver knowledge, attitudes or confidence. Studies assessing medication support interventions at TiC would benefit from improved reporting on development, characteristics of the intervention and usual care comparators, and training administered. As well, active involvement of people with higher medication-related needs, such as those with low literacy, language barriers, and disabilities, is highly encouraged.

\section{Data Sharing Statement}

All search strategies are included in Appendix 1 and included studies are cited herein. Citations for excluded studies are available on reasonable request from the corresponding author.

\section{Acknowledgments}

The authors wish to thank Allie Dai, Dana Green-Campbell, Sanket Patel, Miles Luke, Clara Korenvain for their contributions to data collection, David Lightfoot for his assistance with reviewing the search strategy, and Melanie Dissanayake for reviewing and editing the manuscript. We also wish to thank our larger investigator team including Audrey Chaput, Michelle Ransom, Angela Gonzales, Tai Huynh, Aisha Lofters, Yona Lunsky, Carol Mulder for their contributions to conceptualization of the study.

\section{Author Contributions}

Conceptualization: LMM, SHG, GBR, SJTG, LJ, KO, ZRY, SV

Formal analysis: SV, ZD, LMM

Investigation: SV, ZD, LMM

Methodology: SV, SHG, ZD, GBR, SJTG, LJ, CM, KO, ZRY, LMM

Project administration: SV, LMM

Supervision: LMM

Visualization: SV, SHG, ZD, GBR, SJTG, LJ, CM, KO, ZRY, LMM

Writing - original draft: SV, ZD, LMM

Writing - reviewing and editing: SV, SHG, ZD, GBR, SJTG, LJ, CM, KO, ZRY, LMM 
All authors contributed to data analysis, drafting or revising the article, have agreed on the journal to which the article will be submitted, gave final approval of the version to be published, and agree to be accountable for all aspects of the work.

\section{Funding}

This work was supported by the Canadian Institutes of Health Research (CIHR) FRN: 163065. Stipends for ZhiDi Deng and Shawn Varghese were partially supported by the Leslie Dan Faculty of Pharmacy, University of Toronto.

\section{Disclosure}

Dr Lisa M McCarthy reports grants from Canadian Institutes for Health Research during the conduct of the study. The authors have declared no potential conflicts of interest.

\section{References}

1. WHO. WHO Global Patient Safety Challenge: Medication Without Harm. WHO. Available from: http://www.who.int/patientsafety/medicationsafety/medication-without-harm-brochure/en/. Accessed April 17, 2021.

2. Kee KW, Char CWT, Yip AYF. A review on interventions to reduce medication discrepancies or errors in primary or ambulatory care setting during care transition from hospital to primary care. J Fam Med Prim Care. 2018;7(3):501-506. doi:10.4103/jfmpc.jfmpc_196_17

3. Wheeler AJ, Scahill S, Hopcroft D, Stapleton H. Reducing medication errors at transitions of care is everyone's business. Aust Prescr. 2018;41 (3):73-77. doi:10.18773/austprescr.2018.021

4. Sultana J, Cutroneo P, Trifirò G. Clinical and economic burden of adverse drug reactions. J Pharmacol Pharmacother. 2013;4(Supp11):S73-S77. doi:10.4103/0976-500X.120957

5. Eibergen L, Janssen MJ, Blom L, et al. Informational needs and recall of in-hospital medication changes of recently discharged patients. Res Soc Admin Pharm. 2018;14(2):146-152.

6. Saqib A, Atif M, Ikram R, Riaz F, Abubakar M, Scahill S. Factors affecting patients' knowledge about dispensed medicines: a Qualitative study of healthcare professionals and patients in Pakistan. PLoS One. 2018;13(6):e0197482. doi:10.1371/journal.pone.0197482

7. Karliner LS, Auerbach A, Nápoles A, Schillinger D, Nickleach D, Pérez-Stable EJ. Language barriers and understanding of hospital discharge instructions. Med Care. 2012;50(4):283-289. doi:10.1097/MLR.0b013e318249c949

8. de Moissac D, Bowen S. Impact of language barriers on quality of care and patient safety for official language minority francophones in Canada. $J$ Patient Exp. 2019;6(1):24-32. doi:10.1177/2374373518769008

9. Coleman EA, Smith JD, Raha D, Min S. Posthospital medication discrepancies: prevalence and contributing factors. Arch Intern Med. $2005 ; 165$ (16):1842-1847. doi:10.1001/archinte.165.16.1842

10. Guilcher SJT, Hogan M-E, Calzavara A, et al. Prescription drug claims following a traumatic spinal cord injury for older adults: a retrospective population-based study in Ontario, Canada. Spinal Cord. 2018;56(11):1059-1068. doi:10.1038/s41393-018-0174-z

11. Sullivan WF, Diepstra H, Heng J, et al. Primary care of adults with intellectual and developmental disabilities: 2018 Canadian consensus guidelines. Can Fam Physician Med Fam Can. 2018;64(4):254-279.

12. Cawthon C, Walia S, Osborn CY, Niesner KJ, Schnipper JL, Kripalani S. Improving care transitions: the patient perspective. J Health Commun. 2012;17(Suppl 3):312-324. doi:10.1080/10810730.2012.712619

13. Hahn-Goldberg S, Chaput A, Rosenberg-Yunger Z, et al. Tool development to improve medication information transfer to patients during transitions of care: a participatory action research and design thinking methodology approach. Res Soc Adm Pharm. 2021. doi:10.1016/j. sapharm.2021.04.002

14. Peters M, Godfrey C, McInerney P, Munn Z, Trico A, Khalil H. Chapter 11: scoping Reviews. In: Aromataris E, Munn Z editors. JBI Manual for Evidence Synthesis. JBI;2020. doi:10.46658/JBIMES-20-12

15. Tricco AC, Lillie E, Zarin W, et al. PRISMA Extension for Scoping Reviews (PRISMA-ScR): checklist and explanation. Ann Intern Med. 2018;169 (7):467-473. doi:10.7326/M18-0850

16. Definition of caregiver - NCI Dictionary of Cancer Terms - National Cancer Institute; 2011. Available from: https://www.cancer.gov/publications/ dictionaries/cancer-terms/def/caregiver. Accessed April 17, 2021.

17. Vira T, Colquhoun M, Etchells E. Reconcilable differences: correcting medication errors at hospital admission and discharge. Qual Saf Health Care. 2006;15(2):122-126. doi:10.1136/qshc.2005.015347

18. Forster AJ, Clark HD, Menard A, et al. Adverse events among medical patients after discharge from hospital. CMAJ Can Med Assoc J J Assoc Medicale Can. 2004;170(3):345-349.

19. Okrainec K, Lau D, Abrams HB, et al. Impact of patient-centered discharge tools: a systematic review. J Hosp Med. 2017;12(2):110-117. doi: $10.12788 / \mathrm{jhm} .2692$

20. Deeks JJ, Dinnes J, D’Amico R, et al. Evaluating non-randomised intervention studies. Health Technol Assess Winch Engl. 2003;7(27):iii-x, 1-173. doi:10.3310/hta7270

21. McGowan J, Sampson M, Salzwedel DM, Cogo E, Foerster V, Lefebvre C. PRESS Peer Review of Electronic Search Strategies: 2015 guideline statement. J Clin Epidemiol. 2016;75:40-46. doi:10.1016/j.jclinepi.2016.01.021

22. Covidence - Better systematic review management. Available from: https://www.covidence.org/. Accessed April 17, 2021.

23. Landis JR, Koch GG. The measurement of observer agreement for categorical data. Biometrics. 1977;33(1):159-174.

24. Liberati A, Altman DG, Tetzlaff J, et al. The PRISMA statement for reporting systematic reviews and meta-analyses of studies that evaluate health care interventions: explanation and elaboration. PLOS Med. 2009;6(7):e1000100. doi:10.1371/journal.pmed.1000100 
25. Moher D, Liberati A, Tetzlaff J, Altman DG, Group TP. Preferred reporting items for systematic reviews and meta-analyses: the PRISMA Statement. PLOS Med. 2009;6(7):e1000097. doi:10.1371/journal.pmed.1000097

26. Witteman HO, Vaisson G, Provencher T, et al. An 11-item measure of user- and human-centered design for personal health tools (UCD-11): development and validation. J Med Internet Res. 2021;23(3):e15032. doi:10.2196/15032

27. Acomb C, Laverty U, Smith H, Fox G, Petty D. Medicines optimisation on discharge. The Integrated Medicines oPtimisAtion on Care Transfer (IMPACT) project: 0171. Int J Pharm Pract. 2013;21:123-124.

28. Al-Hashar A, Al-Zakwani I, Eriksson T, et al. Impact of medication reconciliation and review and counselling, on adverse drug events and healthcare resource use. Int J Clin Pharm. 2018;40(5):1154-1164. doi:10.1007/s11096-018-0650-8

29. Anderegg SV, Demik DE, Carter BL, et al. Acceptance of recommendations by inpatient pharmacy case managers: unintended consequences of hospitalist and specialist care. Pharmacotherapy. 2013;33(1):11-21. doi:10.1002/phar.1164

30. Bailey JE, Surbhi S, Wan JY, et al. Effect of intensive interdisciplinary transitional care for high-need, high-cost patients on quality, outcomes, and costs: a Quasi-experimental study. J Gen Intern Med. 2019;34(9):1815-1824. doi:10.1007/s11606-019-05082-8

31. Baky V, Moran D, Warwick T, et al. Obtaining a follow-up appointment before discharge protects against readmission for patients with acute coronary syndrome and heart failure: a quality improvement project. Int J Cardiol. 2018;257:12-15. doi:10.1016/j.ijcard.2017.10.036

32. Balling L, Erstad BL, Weibel K. Impact of a transition-of-care pharmacist during hospital discharge. J Am Pharm Assoc JAPhA. 2015;55(4):443448. doi:10.1331/JAPhA.2015.14087

33. Barnason S, Zimmerman L, Hertzog M, Schulz P. Pilot testing of a medication self-management transition intervention for heart failure patients. West J Nurs Res. 2010;32(7):849-870. doi:10.1177/0193945910371216

34. Bell SP, Schnipper JL, Goggins K, et al. Effect of pharmacist counseling intervention on health care utilization following hospital discharge: a randomized control trial. J Gen Intern Med. 2016;31(5):470-477. doi:10.1007/s11606-016-3596-3

35. Bolas H, Brookes K, Scott M, McElnay J. Evaluation of a hospital-based community liaison pharmacy service in Northern Ireland. Pharm World Sci PWS. 2004;26(2):114-120. doi:10.1023/b:phar.0000018601.11248.89

36. Bonetti AF, Bagatim BQ, Mendes AM, et al. Impact of discharge medication counseling in the cardiology unit of a tertiary hospital in Brazil: a randomized controlled trial. Clin Sao Paulo Braz. 2018;73:e325. doi:10.6061/clinics/2018/e325

37. Budiman T, Snodgrass K, Komatsu Chang A. Evaluation of pharmacist medication education and post-discharge follow-up in reducing readmissions in patients with ST-Segment Elevation Myocardial Infarction (STEMI). Ann Pharmacother. 2016;50(2):118-124. doi:10.1177/ 1060028015620425

38. Cabilan CJ, Boyde M, Currey E. The effectiveness of pharmacist- led discharge medication counselling in the emergency department (ExPLAIN): a pilot quasi-experimental study. Patient Educ Couns. 2019;102(6):1157-1163. doi:10.1016/j.pec.2019.01.020

39. Chakravarthy B, Somasundaram S, Mogi J, et al. Randomized pilot trial measuring knowledge acquisition of opioid education in emergency department patients using a novel media platform. Subst Abuse. 2018;39(1):27-31. doi:10.1080/08897077.2017.1375061

40. Chan BS, Mills N, Chiew A. Does physician education and factsheet impact on safe opioid use in emergency patients? Can J Addict. 2018;9(2):2633. doi:10.1097/CXA.0000000000000019

41. Chedepudi PR, Mounika O, Chandrika G, et al. Impact of pharmacist led anticoagulation monitoring and patient education on oral anticoagulation therapy with acenocoumarol. Asian J Pharm Clin Res. 2017:314-317. doi:10.22159/ajpcr.2017.v10i10.16459.

42. Christy S, Sin B, Gim S. Impact of an integrated pharmacy transitions of care pilot program in an urban hospital. J Pharm Pract. 2016;29(5):490494. doi:10.1177/0897190014568674

43. Cordasco KM, Asch SM, Bell DS, et al. A low-literacy medication education tool for safety-net hospital patients. Am J Prev Med. 2009;37(6 Suppl 1):S209-216. doi:10.1016/j.amepre.2009.08.018

44. Côté J, Godin G, Ramirez-Garcia P, et al. Virtual intervention to support self-management of antiretroviral therapy among people living with HIV. $J$ Med Internet Res. 2015;17(1):e6. doi:10.2196/jmir.3264

45. Davis KK, Mintzer M, Dennison Himmelfarb CR, Hayat MJ, Rotman S, Allen J. Targeted intervention improves knowledge but not self-care or readmissions in heart failure patients with mild cognitive impairment. Eur J Heart Fail. 2012;14(9):1041-1049. doi:10.1093/eurjhf/hfs096

46. Sáez de la Fuente J, Granja Berná V, Lechuga Vázquez P, et al. [Efficiency of the information given at discharge and adherence of polymedicated patients]. Farm Hosp Organo of Expresion Cient Soc Espanola Farm Hosp. 2011;35(3):128-134. Spanish. doi:10.1016/j.farma.2010.06.005

47. Ducharme FM, Zemek RL, Chalut D, et al. Written action plan in pediatric emergency room improves asthma prescribing, adherence, and control. Am J Respir Crit Care Med. 2011;183(2):195-203. doi:10.1164/rccm.201001-0115OC

48. Feldmann JD, Otting RI, Otting CM, Witry MJ. A community pharmacist-led service to facilitate care transitions and reduce hospital readmissions. $J$ Am Pharm Assoc JAPhA. 2018;58(1):36-43. doi:10.1016/j.japh.2017.09.004

49. Hyrkas K, Wiggins M. A comparison of usual care, a patient-centred education intervention and motivational interviewing to improve medication adherence and readmissions of adults in an acute-care setting. J Nurs Manag. 2014;22(3):350-361. doi:10.1111/jonm.12221

50. Jiang R, Chen M, Li Y. Effectiveness of picture description education on compliance behaviors of diabetics in western Sichuan district. Int J Nurs Sci. 2016;3(3):229-234. doi:10.1016/j.ijnss.2016.08.008

51. Jones CD, Anthony A, Klein MD, et al. The effect of a pharmacist-led multidisciplinary transitions-of-care pilot for patients at high risk of readmission. J Am Pharm Assoc JAPhA. 2018;58(5):554-560. doi:10.1016/j.japh.2018.05.008

52. Kaestli LZ, Noble S, Combescure C, et al. Drug information leaflets improve parental knowledge of their child's treatment at paediatric emergency department discharge. Eur J Hosp Pharm. 2016;23(3):151-155. doi:10.1136/ejhpharm-2015-000776

53. Kapoor A, Landyn V, Wagner J, et al. Supplying pharmacist home visit and anticoagulation professional consultation during transition of care for patients with venous thromboembolism. J Patient Saf. 2020;16(4):e367-e375. doi:10.1097/PTS.0000000000000571

54. Khonsari S, Subramanian P, Chinna K, Latif LA, Ling LW, Gholami O. Effect of a reminder system using an automated short message service on medication adherence following acute coronary syndrome. Eur J Cardiovasc Nurs J Work Group Cardiovasc Nurs Eur Soc Cardiol. 2015;14 (2):170-179. doi:10.1177/1474515114521910

55. Lam P, Elliott RA, George J. Impact of a self-administration of medications programme on elderly inpatients' competence to manage medications: a pilot study. J Clin Pharm Ther. 2011;36(1):80-86. doi:10.1111/j.1365-2710.2009.01157.x

56. Leguelinel-Blache G, Dubois F, Bouvet S, et al. Improving Patient's Primary Medication Adherence: the Value of Pharmaceutical Counseling. Medicine (Baltimore). 2015;94(41):e1805. doi:10.1097/MD.0000000000001805 
57. Li H, Guffey W, Honeycutt L, Pasquale T, Rozario NL, Veverka A. Incorporating a pharmacist into the discharge process: a unit-based transitions of care pilot. Hosp Pharm. 2016;51(9):744-751. doi:10.1310/hpj5109-744

58. Louis-Simonet M, Kossovsky MP, Sarasin FP, et al. Effects of a structured patient-centered discharge interview on patients' knowledge about their medications. Am J Med. 2004;117(8):563-568. doi:10.1016/j.amjmed.2004.03.036

59. Lingyun L, Cynthia J, de Leon N, Alberta W, Donald C, Freny M. Impact of a multi-disciplinary heart failure post-discharge management clinic on medication adherence. J Am Coll Cardiol. 2014;63(12_Supplement):A539-A539. doi:10.1016/S0735-1097(14)60539-8

60. Luder HR, Frede SM, Kirby JA, et al. TransitionRx: impact of community pharmacy postdischarge medication therapy management on hospital readmission rate. J Am Pharm Assoc JAPhA. 2015;55(3):246-254. doi:10.1331/JAPhA.2015.14060

61. Manning DM, O’Meara JG, Williams AR, et al. 3D: a tool for medication discharge education. Qual Saf Health Care. 2007;16(1):71-76. doi:10.1136/qshc.2006.018564

62. Marusic S, Gojo-Tomic N, Erdeljic V, et al. The effect of pharmacotherapeutic counseling on readmissions and emergency department visits. Int $J$ Clin Pharm. 2013;35(1):37-44. doi:10.1007/s11096-012-9700-9

63. Marušić S, Meliš P, Lucijanić M, et al. Impact of pharmacotherapeutic education on medication adherence and adverse outcomes in patients with type 2 diabetes mellitus: a prospective, randomized study. Croat Med J. 2018;59(6):290-297.

64. McCarthy DM, Wolf MS, McConnell R, et al. Improving patient knowledge and safe use of opioids: a randomized controlled trial. Acad Emerg Med off J Soc Acad Emerg Med. 2015;22(3):331-339. doi:10.1111/acem.12600

65. Miller DE, Roane TE, McLin KD. Reduction of 30-day hospital readmissions after patient-centric telephonic medication therapy management services. Hosp Pharm. 2016;51(11):907-914. doi:10.1310/hpj5111-907

66. Moye PM, Chu PS, Pounds T, Thurston MM. Impact of a pharmacy team-led intervention program on the readmission rate of elderly patients with heart failure. Am J Health-Syst Pharm. 2018;75(4):183-190. doi:10.2146/ajhp170256

67. Murphy JA, Schroeder MN, Rarus RE, Yakubu I, McKee SOP, Martin SJ. Implementation of a cardiac transitions of care pilot program: a prospective study of inpatient and outpatient clinical pharmacy services for patients with heart failure exacerbation or acute myocardial infarction. $J$ Pharm Pract. 2019;32(1):68-76. doi:10.1177/0897190017743129

68. Oliveira-Filho AD, Morisky DE, Costa FA, Pacheco ST, Neves SF, Lyra-Jr DP. Improving post-discharge medication adherence in patients with CVD: a pilot randomized trial. Arq Bras Cardiol. 2014;103(6):502-512. doi:10.5935/abc.20140151

69. Olives TD, Patel RG, Thompson HM, Joing S, Miner JR. Seventy-two-hour antibiotic retrieval from the ED: a randomized controlled trial of discharge instructional modality. Am J Emerg Med. 2016;34(6):999-1005. doi:10.1016/j.ajem.2016.02.046

70. Phatak A, Prusi R, Ward B, et al. Impact of pharmacist involvement in the transitional care of high-risk patients through medication reconciliation, medication education, and postdischarge call-backs (IPITCH Study). J Hosp Med. 2016;11(1):39-44. doi:10.1002/jhm.2493

71. Press VG, Arora VM, Trela KC, et al. Effectiveness of interventions to teach metered-dose and diskus inhaler techniques. A randomized trial. Ann Am Thorac Soc. 2016;13(6):816-824. doi:10.1513/AnnalsATS.201509-603OC

72. Renaudin P, Baumstarck K, Daumas A, et al. Impact of a pharmacist-led medication review on hospital readmission in a pediatric and elderly population: study protocol for a randomized open-label controlled trial. Trials. 2017:18. doi:10.1186/s13063-017-1798-6.

73. Salmany SS, Ratrout L, Amireh A, et al. The impact of pharmacist telephone calls after discharge on satisfaction of oncology patients: a randomized controlled study. J Oncol Pharm Pract off Publ Int Soc Oncol Pharm Pract. 2018;24(5):359-364. doi:10.1177/1078155217709616

74. Sanii Y, Torkamandi H, Gholami K, Hadavand N, Javadi M. Role of pharmacist counseling in pharmacotherapy quality improvement. J Res Pharm Pract. 2016;5(2):132-137. doi:10.4103/2279-042X.179580

75. Sarangarm P, London MS, Snowden SS, et al. Impact of pharmacist discharge medication therapy counseling and disease state education: pharmacist Assisting at Routine Medical Discharge (project PhARMD). Am J Med Qual off J Am Coll Med Qual. 2013;28(4):292-300. doi:10.1177/1062860612461169

76. Schwalm J-D, Ivers NM, Natarajan MK, et al. Cluster randomized controlled trial of Delayed Educational Reminders for Long-term Medication Adherence in ST-Elevation Myocardial Infarction (DERLA-STEMI). Am Heart J. 2015;170(5):903-913. doi:10.1016/j.ahj.2015.08.014

77. Send AFJ, Schwab M, Gauss A, Rudofsky G, Haefeli WE, Seidling HM. Pilot study to assess the influence of an enhanced medication plan on patient knowledge at hospital discharge. Eur J Clin Pharmacol. 2014;70(10):1243-1250. doi:10.1007/s00228-014-1723-9

78. Shaver A, Morano M, Pogodzinski J, Fredrick S, Essi D, Slazak E. Impact of a community pharmacy transitions-of-care program on 30-day readmission. J Am Pharm Assoc JAPhA. 2019;59(2):202-209. doi:10.1016/j.japh.2018.10.011

79. Shull MT, Braitman LE, Stites SD, DeLuca A, Hauser D. Effects of a pharmacist-driven intervention program on hospital readmissions. Am $J$ Health-Syst Pharm. 2018;75(9):e221-e230. doi:10.2146/ajhp170287

80. Singh S, Clarke C, Lawendy AR, et al. First Place: a prospective, randomized controlled trial of the impact of written discharge instructions for postoperative opioids on patient pain satisfaction and on minimizing opioid risk exposure in orthopaedic surgery. Curr Orthop Pract. 2018;29 (4):292-296.

81. Sinha S, Dillon J, Dargar SK, et al. What to expect that you're not expecting: a pilot video education intervention to improve patient self-efficacy surrounding discharge medication barriers. Health Informatics J. 2019;25(4):1595-1605. doi:10.1177/1460458218796644

82. Smith L Reducing Acute Myocardial Infarction Readmissions [dissertation]. USA: Grand Canyon University, 2017.

83. Tuttle KR, Alicic RZ, Short RA, et al. Medication therapy management after hospitalization in CKD: a randomized clinical trial. Clin J Am Soc Nephrol CJASN. 2018;13(2):231-241. doi:10.2215/CJN.06790617

84. Vuong T, Marriott JL, Kong DCM, Siderov J. Implementation of a community liaison pharmacy service: a randomised controlled trial. Int $J$ Pharm Pract. 2008;16(3):127-135. doi:10.1211/ijpp.16.3.0002

85. Walker PC, Bernstein SJ, Jones JNT, et al. Impact of a pharmacist-facilitated hospital discharge program: a quasi-experimental study. Arch Intern Med. 2009;169(21):2003-2010. doi:10.1001/archinternmed.2009.398

86. Zerafa N, Zarb Adami M, Galea J. Impact of drugs counselling by an undergraduate pharmacist on cardiac surgical patient's compliance to medicines. Pharm Pract. 2011;9(3):156-161.

87. Tomlinson J, Cheong VL, Fylan B, et al. Successful care transitions for older people: a systematic review and meta-analysis of the effects of interventions that support medication continuity. Age Ageing. 2020;49(4):558-569.

88. Daliri S, Boujarfi S, El Mokaddam A, et al. Medication-related interventions delivered both in hospital and following discharge: a systematic review and meta-analysis. BMJ Qual Saf. 2021;30(2):146-156. 
89. Hoffmann T, Glasziou P, Boutron I, et al. Die TIDieR Checkliste und Anleitung - ein Instrument für eine verbesserte Interventionsbeschreibung und Replikation [Better Reporting of Interventions: template for Intervention Description and Replication (TIDieR) Checklist and Guide]. Gesundheitswesen. 2016;78(03):175-188. doi:10.1055/s-0041-111066

90. Hahn-Goldberg S, Okrainec K, Huynh T, Zahr N, Abrams H. Co-creating patient-oriented discharge instructions with patients, caregivers, and healthcare providers. $J$ Hosp Med. 2015;10(12):804-807. doi:10.1002/jhm.2444

91. Huynh TM Design by exception: outliers, misfits and the design of extraordinary healthcare [dissertation]. Canada: OCAD University; $2015: 70$.

92. O'Cathain A, Croot L, Duncan E, et al. Guidance on how to develop complex interventions to improve health and healthcare. BMJ Open. 2019;9: e029954. doi:10.1136/bmjopen-2019-029954

93. Hahn-Goldberg S, Huynh T, Chaput A, et al. Implementation, spread and impact of the Patient Oriented Discharge Summary (PODS) across Ontario hospitals: a mixed methods evaluation. BMC Health Serv Res. 2021;21(1):361. doi:10.1186/s12913-021-06374-8

94. WHO. Rapid Reviews to Strengthen Health Policy and Systems: A Practical Guide. WHO. Available from: http://www.who.int/alliance-hpsr/ resources/publications/rapid-review-guide/en/. Accessed April 17, 2021.

\section{Publish your work in this journal}

Patient Preference and Adherence is an international, peer-reviewed, open access journal that focusing on the growing importance of patient preference and adherence throughout the therapeutic continuum. Patient satisfaction, acceptability, quality of life, compliance, persistence and their role in developing new therapeutic modalities and compounds to optimize clinical outcomes for existing disease states are major areas of interest for the journal. This journal has been accepted for indexing on PubMed Central. The manuscript management system is completely online and includes a very quick and fair peer-review system, which is all easy to use. Visit http://www.dovepress.com/testimonials.php to read real quotes from published authors.

Submit your manuscript here: https://www.dovepress.com/patient-preference-and-adherence-journal 